Michael A. Arbib (2016): Towards a Computational Comparative Neuroprimatology: Framing the Language-Ready Brain. To appear in Physics of Life Reviews with commentaries and a response.

\title{
Towards a Computational Comparative Neuroprimatology: Framing the Language-Ready Brain Michael A. Arbib
}

Abstract: We make the case for developing a Computational Comparative Neuroprimatology to inform the analysis of the function and evolution of the human brain.

First, we update the mirror system hypothesis on the evolution of the language-ready brain by (i) modeling action and action recognition and opportunistic scheduling of macaque brains to hypothesize the nature of the last common ancestor of macaque and human (LCA-m); and then (ii) introduce dynamic brain modeling to show how apes could acquire gesture through ontogenetic ritualization, hypothesizing the nature of evolution from LCA-m to the last common ancestor of chimpanzee and human (LCA-c). We then (iii) hypothesize the role of imitation, pantomime, protosign and protospeech in biological and cultural evolution from LCA-c to Homo sapiens with a language-ready brain.

Second, we suggest how cultural evolution in Homo sapiens led from protolanguages to full languages with grammar and compositional semantics.

Third, we assess the similarities and differences between the dorsal and ventral streams in audition and vision as the basis for presenting and comparing two models of language processing in the human brain: A model of (i) the auditory dorsal and ventral streams in sentence comprehension; and (ii) the visual dorsal and ventral streams in defining "what language is about" in both production and perception of utterances related to visual scenes provide the basis for (iii) a first step towards a synthesis and a look at challenges for further research.

Keywords: action; action recognition; ape gesture; audition, computational neuroscience; cultural evolution; dorsal stream; dyadic brain modeling; evolution; language, language-ready 
brain; macaque brain; mirror neuron; mirror system hypothesis; neurolinguistics; neurolinguistics; neuroprimatology; primatology; ventral stream; vision

\section{Introduction}

This review includes some familiar tropes on the mirror system hypothesis (MSH) of the evolution of the human language-ready brain as a basis for expanding upon prior research. Since some readers will be familiar with the background material, Section 1.1. explains how this review relates to prior publications. The rest of this section then provides "background to the background" or otherwise sets the stage for what follows.

Section 1.2. explains a parallel between vision and auditory pathways in the primate brain both have dorsal and ventral streams radiating from the respective primary sensory cortices. The visual streams will play a key role for our base model of the macaque brain (and thus for our hypothesized brain for LCA-m, the last common ancestor of macaque and human) in Section 2.1; we will then build on this in assessing the implications of MSH for understanding the modern human brain in Section 4.1, and offer further notions based on the auditory and visual streams in Sections 4.2 and 4.3 , respectively.

A number of the brain models in this review are based on modelling the interaction of biologically plausible neural networks localized in specific brain regions. Section 1.3 introduces a complementary approach, schema theory, based on the competition and cooperation of distributed processes called schemas. Once a schema-theoretic model of a cognitive process has been obtained, one may seek a (possibly alternative) description in which subschemas may be linked to the activity of specific neural networks in the brain. We consider two examples of schema-based models. For the first, reaching and grasping, such an implementation has proved possible (see Section 2.1); for the second, visual scene interpretation, it has not.

Most brain modeling focuses on one brain at a time, seeking to delineate neural processes in perception, memory, action, and so on. However, an emerging focus on social neuroscience (of which language as implicated in conversation is surely a crucial part) requires that we develop dyadic brain modeling - modeling the brains of two creatures as they interact with each other, so that the action of one affects the perception of the other and so the cycle of interactions continues, with both brains changing in the process. This is the focus of Section 1.4. Unfortunately, a theory of conversation is beyond the scope of this review, but we do offer an 
example of dyadic brain modeling in Section 2.2, suggesting how interaction between two apes may support the emergence of novel gestures.

Section 1.5 introduces the key notion of computational comparative neuroprimatology. The primates we consider are monkeys, apes, and humans. By comparing behavior and communication across diverse species of these primates, we can stimulate the development of schema-theoretic models both of what is shared across species (a candidate for hypotheses on the last common ancestor) and what is different (stimulating evolutionary hypotheses, and the challenge of teasing apart biological and cultural evolution). This comparative primatology becomes computational comparative neuroprimatology when computational models of brain mechanisms are thrown into the mix.

A human language (such as English and Swahili, but not music) is a system which combines a lexicon with a grammar which supports a compositional semantics, i.e., it supports an unbounded set of propositions whose meaning can be inferred from the meanings of words through the way the grammar combines them. (This is a useful approximation - for example, context clearly affects our understanding of what is said.) Since no nonhuman primate has language in this sense, it is a plausible assumption that somewhere in our evolutionary tree we had ancestors with a system more open-ended than the communication systems of other species yet far less flexible than modern human languages. Section 1.6 introduces the notion of "protolanguage" in the sense of such an intermediate system.

Finally, we note that linguists have developed many different ways of characterizing grammars. No satisfactory characterization yet exists, especially when the challenges of understanding performance and brain mechanisms are taken into account. Section 1.7 introduces construction grammar as a potentially useful framework for our research, while noting that it comes in many "flavors" so that, even here, the choice of an optimal framework for neurolinguistics is very much an open challenge.

\subsection{How this Review Relates to Prior Publications}

My concern with linking language to the brain goes back at least to Arbib and Caplan (1979) and the follow-up edited volume (Arbib, Caplan, \& Marshall, 1982), but the main impetus for the present review can be traced back to two papers that I wrote with Giacomo Rizzolatti (Arbib \& Rizzolatti, 1997; Rizzolatti \& Arbib, 1998) which introduced the Mirror System Hypothesis (MSH). Noting that 
(i) area F5 of macaque brains contains mirror neurons responsive both during action and execution of similar hand movements; neurons active only during execution but not observation are called canonical neurons;

(ii) brain imaging (e.g., Grafton, Arbib, Fadiga, \& Rizzolatti, 1996) reveals a human mirror system for grasping - i.e., a brain region activated for both grasping and observation of grasping - in or near Broca's area, an area traditionally viewed as involved in speech production;

(iii) F5 seems homologous to (part of) Broca's area in humans, and

(iv) aphasia of signed, and not just spoken, languages may result from lesions "embracing" Broca's area (Poizner, Klima, \& Bellugi, 1987)

we argued not only that one should associate Broca's area and its environs with multimodal language production rather than with speech alone but further for the importance of manual gesture in the evolution of the human capacity for language, with the mirror system integrating action and execution of hand actions providing crucial evolutionary support for mechanisms supporting language parity in the human brain: the fact that the meaning the speaker (or signer) intends to express can (often) become the meaning that the hearer (observer) gains access to as a result.

Different brain regions (not individual neurons) may be implicated in the human brain as mirror systems for different classes of actions and even emotions, and many researchers have attributed high level cognitive functions such as empathy, imitation, intention attribution and language to human mirror regions (Rizzolatti \& Sinigaglia, 2008). However, monkeys do not imitate or learn language. Thus, any account of the role of human mirror systems in imitation and language must - as MSH does - include an account of the evolution not only of mirror systems, but also of their interaction with more extended systems beyond the mirror system within the human brain.

Unavoidably, the initial papers offered an incomplete and partially erroneous account and so I published a range of papers - perhaps the best known is (Arbib, 2005), but many others extend the analysis - which sought to correct and extend that initial account. This process had its initial culmination in a book called How the Brain Got Language: The Mirror System Hypothesis (Arbib, 2012). However, it was never my view that this account was the endpoint of the investigation, and I was thus delighted when David Kemmerer organized a special issue of 
Language and Cognition which combined a précis of the book (Arbib, 2013b) with 12 vigorous commentaries (from experts in anthropology, apraxia, archeology, linguistics, neuroanatomy, neuroimaging, neurophysiology, neuropsychology, primatology, sign language emergence and sign language neurolinguistics) and my reply (Arbib, 2013a). Readers may well find the variety of opinions pro and con MSH stimulating as a basis for further research.

The aim of this review, then, is to carry the story forward, by presenting material that has been developed since the publication of How the Brain Got Language, or got short shrift there. However, since I cannot (and do not wish to) assume all readers of this review know the book or key papers on which it builds, the present review will include summaries of the necessary background.

The shape of this review was determined in developing an invited plenary lecture "Evolving the Language-Ready Brain: The Case for a Comparative Neuroprimatology" for the EVOLANG X conference on language evolution held at the University of Vienna in 2014. The central theme was to emphasize an approach to understanding of the evolution of the human language-ready brain based on the comparative study of action-oriented perception, communication and language in monkeys, apes and humans (comparative neuroprimatology). It also stressed the importance of modeling, whether in terms of networks of schemas or neurons; thus the title of this review, Towards a Computational Comparative Neuroprimatology: Framing the LanguageReady Brain. The talk introduced three themes developed in this review: "classic" neural models of macaque brain mechanisms for generating grasps of objects and for mirror neurons; a lifting of these models to assess how apes might learn novel communicative gestures via ontogenetic ritualization; and a new approach to modeling the processes involved in generating descriptions of visual scenes and in comprehending such utterances. Some of the material was sketched in the book, but there were new results and challenges to report; a preliminary review of these was provided by Arbib, Gasser, and Barrès (2014) in a paper that was in large part a tribute to the pioneering work of Marc Jeannerod in placing perception in an action-oriented framework. The first draft of the present review also sought to extend the analysis of the dorsal \& ventral pathways (for vision) presented in Arbib (2010).

Two things happened to cause me to add further material in preparing this final version of the review: 
1) The draft had a single reviewer, who offered no comments on either the comparative neuroprimatology or the computational modeling that I viewed as the key foci of the review, but instead voiced three concerns:

(a) it only mentioned in passing the quite extensive literature on the neurobiology of language and its close relationship with the auditory system;

(b) it was mainly about vision and action, rather than about language;

(c) the fact that the transition from "protolanguage" to language was outside of the scope of the draft raised the question of how much about language this account, in fact, explains.

I plead guilty to (a) and will return to it in discussing point (2) below.

Perhaps the reviewer simply missed the point on $(b)$ - which is that the production of spoken or signed language is a form of (social) action, and that the comprehension of language is thus a form of action-oriented perception. Moreover, much of what we talk about is linked to our (in great part visual) perception of the social and physical environment. Or perhaps the reviewer views linguistics as the study of strings of words that are or are not "well-formed." I hope the reader will agree that point (b) is mistaken for all those who wish to relate the use of language to processes in the brain during performance.

Objection (c) seems unreasonable - every paper has a limited scope. Indeed, Chapters 10 through 13of How the Brain Got Language do offer an account (partial and provisional, of course) of the transition from "protolanguage" to language. However, in the hope of helping readers and commentators see how the core of this review is indeed relevant to language, I have added a synopsis of those chapters, with a few extra comments thrown in.

2) My paper cited above (Arbib, 2010) focuses on the dorsal and ventral pathways for vision. As noted in How the Brain Got Language (pp.118-119), early work on these two visual pathways had inspired later work by Rauschecker and his colleagues delimiting two auditory pathways - but I had little to say about the relevance of this to auditory processing of speech beyond noting the work of Hickok and Poeppel (2004) relating the auditory dorsal and ventral streams to speech comprehension. I was thus delighted when Bornkessel-Schlesewsky and Schlesewsky (2013) outlined a "new dorsal-ventral stream model of sentence comprehension," but this time based on the auditory streams. I set about an attempt to integrate these two accounts to provide a more comprehensive view of neural processing of language. Given this history, it seemed to me that the best response to critique (a) above, "[the review] only mentions in passing 
the quite extensive literature on the neurobiology of language and its close relationship with the auditory system" was to edit my draft rapprochement of the auditory and visual streams into the present review. However, the coverage of the neurolinguistics literature here remains highly limited. My Handbook paper (Arbib, 2015) offers a somewhat unorthodox account of neurolinguistics, but it also offers pointers to the "mainstream" literature, while noting that the accounts on offer remain somewhat incompatible and that an adequate foundation for neurolinguistics remains to be found.

One more comment: the focus of this review is on how comparative neuroprimatology may enrich our quest to understand the evolutionary stages that led to language, not on language per se - I offer just enough of our current work on neurolinguistics to clarify further discussion of "what it is that evolved."

\subsection{Dorsal and Ventral Streams in Vision and Audition}

As is well known, one may contrast the dorsal "how" path from visual cortex through parietal cortex with the ventral "what" path through inferotemporal (IT) cortex based on studies of two patients as well as macaque neurophysiology: DF, with a ventral lesion, is a visual form agnosic who does not have access to conscious declarative information about form (temporally mediated) but can use form information to guide grasping (Jakobson, Archibald, Carey, \& Goodale, 1991). AT, with a dorsal lesion can pantomime grasp but cannot preshape based on current form information (Jeannerod, Decety, \& Michel, 1994). (In Section 4 we shall seek to integrate a view of the role of these paths in language with an account of the role of dorsal and ventral paths for audition.) The data suggest one cannot pantomime or verbalize an affordance (in this case a visual indication of the motor parameters for a possible grasping action) if it is only available via the dorsal path; but rather one needs a "recognition of the object" (IT) with which attributes can be associated before one can express them.

- Dorsal stream: Patient DF has a ventral lesion but intact parietal stream and is able to use vision of the size or orientation of an object to preshape her hand. We conclude that parietal affordances map to motor parameters for preshape.

- Ventral stream: Patient AT has a dorsal lesion but intact ventral stream and is able to use vision of the size or orientation of an object to describe this attribute but not preshape her hand. We conclude that IT perception of an attribute of an object can support pantomime or verbal description of that attribute. 
This puts speech and pantomime on one side of the divide, and object-based action on the other. However, in Section 4.1, we will refine this story somewhat: suggesting that the articulation of language rests more on the dorsal pathway while the meaning of language rests more on the ventral pathway.

\subsection{Schema Theory: Reaching, Grasping and Scene Interpretation}

The work of Jeannerod and Biguer (1982) on human preshaping of the hand while reaching to grasp was the basis for an early coordinated control program (Arbib, 1981) integrating perceptual schemas for extracting relevant properties of an object with the motor schemas that coordinate arm and hand movements in reaching to and grasping the object. Different perceptual schemas provide input for the motor schemas for the control of reaching grasping (controlling the hand to conform to the object). Reaching was hypothesized to involve a ballistic phase followed by a feedback phase, with the transition between the two also activating the transition within the motor schema for grasping from a preshape subschema to an enclose subschema.

In Section 4.3, we extend earlier work (Arbib, Conklin, \& Hill, 1987; Arbib \& Lee, 2008) applying schema theory to language processing. As background for this, we now examine an early use of schema theory to address the following question: "How do we recognize a novel visual scene?" The short answer will be "By associating 'schema instances' with regions of the scene, with the instance linked to a region corresponding to the schema most activated for that region." The VISIONS system (Arbib, 1989, Sec. 5.3; Draper, Collins, Brolio, Hanson, \& Riseman, 1989; Hanson \& Riseman, 1978) was a scene-understanding system that could move from a color photograph to a labeling of the various objects contained in the scene. It was implemented on a serial computer but the underlying computational architecture offered useful insights into the way I believe the brain operates, via cooperative computation, the competition and cooperation of a multitude of schema instances - supported in turn by the inhibitory and excitatory interactions of a far larger number of neurons. However, there are areas of cognition where brain models that can be constructed satisfactorily at the schema network level do not transfer well to biologically realistic neural network models (despite decades of research since the lament of Arbib, 1988). Thus the models of high-level vision (here) and language production and comprehension (Section 4.3) that we review in this review are both schema-theoretic, and their linkage to more data from neuroscience - and thus their modification and/or elaboration in a continuing modeling-experimentation cycle (where "experimentation" includes clinical studies 
of humans and field work to observe non-human primate behavior) - provide important challenges for both modeling and experimentation.

Before the schemas in VISIONS can operate, low-level processes take an image of an outdoor visual scene and extract an intermediate representation - including contours and surfaces tagged with features such as color, texture, shape, size and location (see the middle panel of Figure 1 (top)). Many of these processes can be modeled with neurobiologically plausible neural networks but models of further processing remain within the realm of schema theory. Perceptual schemas process different features of the intermediate representation to form confidence values for the presence of objects like houses, walls and trees. The knowledge required for interpretation is stored in long-term memory as a network of schemas, while the state of interpretation of the particular scene unfolds in working memory as a network of schema instances. Note that this visual working memory is not defined in terms of recency (as in very short term memory) but rather in terms of continuing relevance. 

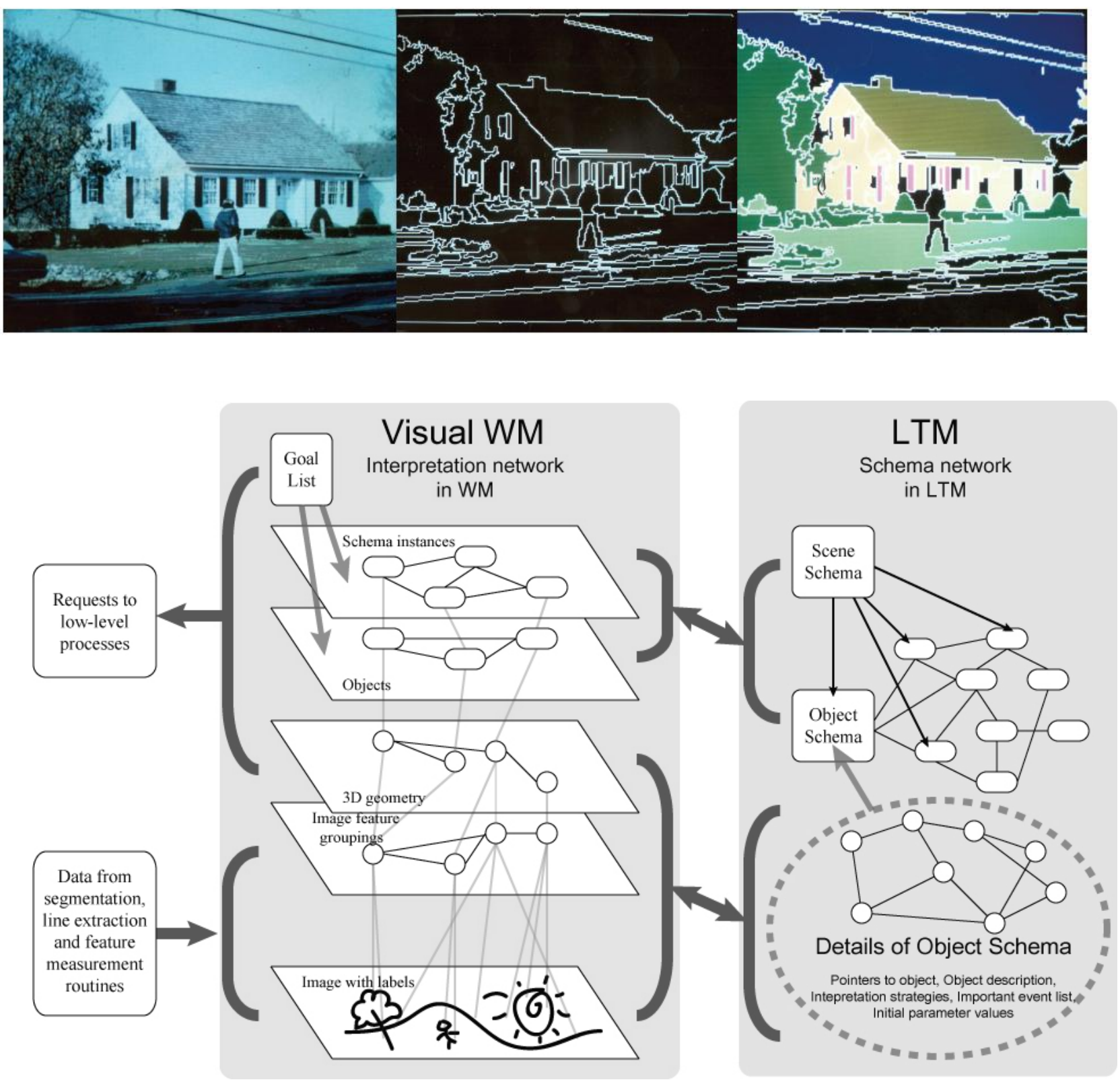

Figure 1. (Top) Segmentation of a scene into candidate regions (middle) provides a bridge between the original image and the interpretation of a scene in VISIONS; the latter proceeds by associating regions of the image with schema instances. In this example, VISIONS classifies regions of the scene as sky, roof, wall, shutter foliage and grass, but leaves other areas uninterpreted. Images courtesy of Allen Hanson. (Bottom) The Visual Working Memory (WM) of VISIONS interprets the current scene by a network of parameterized instances of schemas from Long Term Memory (LTM). These schema instances are linked to the visual world via the intermediate database that offers an updatable analysis of the division of the world into regions that are candidates for interpretation as agents and objects, possibly in relation with each other. 
Interpretation of a novel scene starts with the data-driven instantiation of several schemas (e.g., a certain range of color and texture might cue an instance of the foliage schema for a certain region of the image). When a schema instance is activated, it is linked with an associated area of the image and an associated set of local variables (See Figure 1 (bottom).). Each schema instance in working memory has an associated confidence level which changes on the basis of interactions with other instances in working memory. Once several schema instances are active, they may instantiate others in a "hypothesis-driven" way (e.g., recognizing what appears to be a roof will activate an instance of the house schema to seek confirming evidence such as the presence of walls in the region below that of the putative roof). Ensuing computation is based on the competition and cooperation of concurrently active schema instances. Once a number of schema instances have been activated, the schema network is invoked to formulate hypotheses, set goals, and then iterate the process of adjusting the activity level of schemas linked to the image until a coherent scene interpretation of (part of) the scene is obtained.

Cooperation (e.g., that between "roof" and "sky" if the spatial relations are appropriate) yields a pattern of "strengthened alliances" between mutually consistent schema instances that allows them to achieve high activity levels to constitute the overall solution of a problem.

As a result of competition (e.g., the same region cannot be both "wall" and sky"), instances which do not meet the evolving consensus lose activity, and thus are not part of this solution (though their continuing subthreshold activity may well affect later behavior). The highly activated instances of perceptual schemas become part of the current working model of the image (as indicated to the right of Figure 1 (top)).

Why hands \& vision as the above examples?

We distinguish praxic actions - physical actions to accomplish tasks in the environment, which includes objects and the bodies of other agents from communicative actions. It should be uncontroversial that brain mechanisms for the control and recognition of manual actions for praxis provided an evolutionary basis for the ability to use the hands for communicative actions. MSH is more controversial, though, in suggesting that this transition provided essential scaffolding for the evolution of brain mechanisms that support speech. We return to this controversy in Section 2.3.

Note, however, that vision should be accorded a prime place in our study of language whether we are studying spoken (audio-vocal) language, or signed (visuo-manual) language, and 
whatever theory of language evolution (if any) we subscribe to. This is because language cannot be fully understood as a matter of word patterns unless we understand how those patterns relate to meaning, and a major source of that meaning comes from our perception of the visual world around us. Thus the emphasis on VISIONS here, and the role of vision in the Template Construction Grammar in Section 4.3. But, of course, this in no way diminishes the importance of the auditory system in our perception of spoken language, a theme we address in Section 4.2.

\subsection{Brains Interacting and Dyadic Brain Modeling}

Much work in cognitive neuroscience focuses on some aspect of the function of a single brain (vision, memory and action, for example), and will use neuroimaging or neuropsychology (brain lesion studies) of specific brain regions (hippocampus, basal ganglia, cerebellum, and various areas of cerebral cortex) as seen in the context of larger networks, focused studies of underlying mechanisms (dynamics of neural networks, Hebbian and reinforcement learning, etc.) or other general strategies of computational neuroscience.

When we turn to language, we are looking at an inherently social phenomenon which (whatever its importance to the cognition of a single individual) evolved to support interactions of two or more people. The basic idea is well conveyed in Figure 2, the semiotic cycle. Here we diagram the interaction of a speaker and a hearer (and the roles are reversed at each turn of the conversation). The diagram shows two forms of interaction:

a) via sensorimotor activity whereby each actor can change the physical state of the world (which includes the bodies of both actors) and thus what each can attend to, which may involve joint attention to varying degrees at different stages of the interaction; and

b) communicative interaction via "utterances," which may be spoken or signed, and may include gestures of voice, face, hands, body posture, and more.

Before proceeding, note - crucially for the build up to a discussion of evolution -- that almost all of this applies to animals in general not just to humans, with the important exception that nonhuman animals cannot master signed or spoken languages and so we might want to replace the term "utterance" by "signal" in the general case. Admittedly, some apes have been taught to use a number of signs based on American Sign Language (ASL) but there is no evidence that they can master grammar to support the ability to combine words in novel ways and have the novel meanings understood, the sine qua non of language in the sense in which we use it in this review (see Section 1.5). 


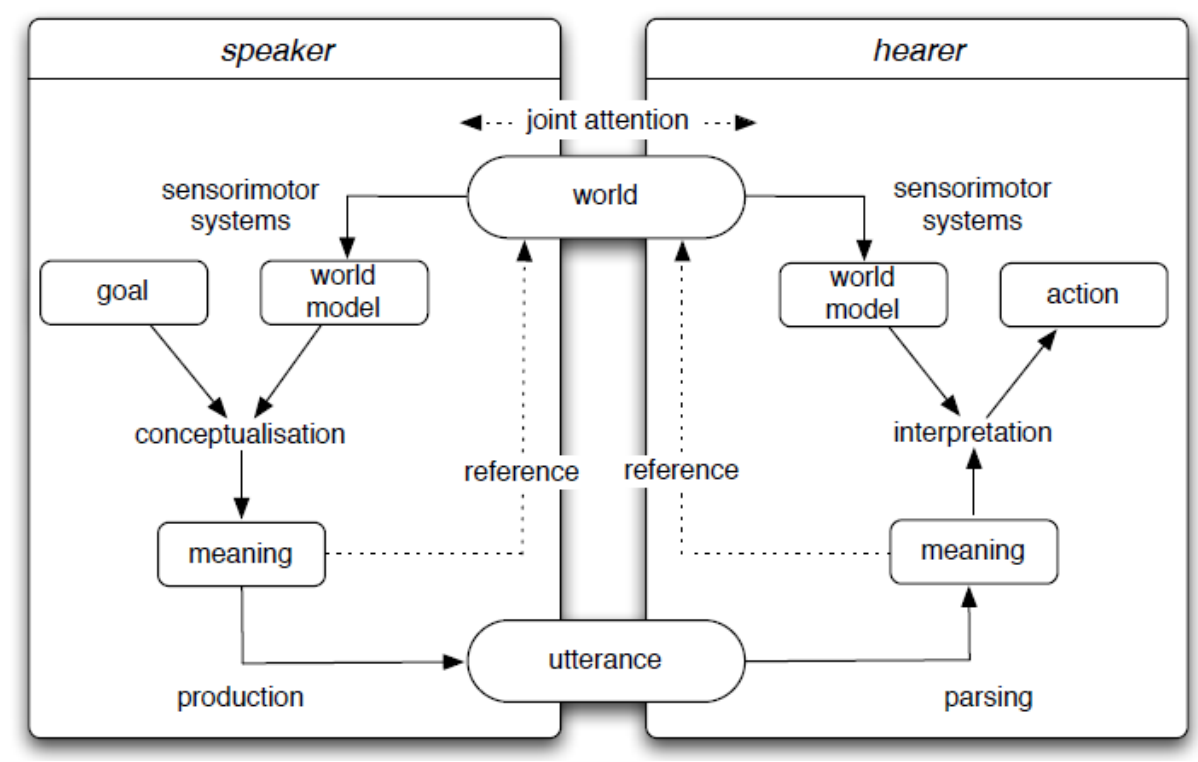

Figure 2. The semiotic cycle (Spranger, 2014) coupling shared attention to and (not shown) interaction with the external world with the sharing of utterances that constitutes communication.

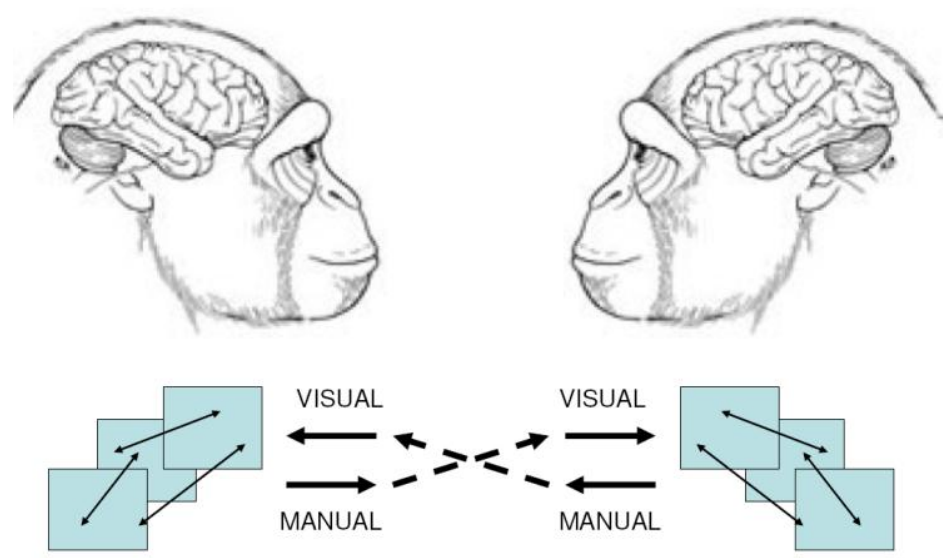

Figure 3: Schematic for Dyadic Brain Modeling. Each brain has the same architecture which it is our challenge to assess on the basis of empirical data and lessons learned from prior modeling. However, each agent's/brain starts in a different state, and thus the actions and learning processes that unfold will differ between the two agents.

Although primate neurophysiology offers a range of studies of mirror neurons in a monkey observing actions of a human or other monkey (including studies that were the basis for the FARS and MNS models reviewed briefly in Section 2.1), little attention has been paid to whether and how mirror neuron activity helps guide the monkey's own behavior (the ACQ model of Section 5 is an exception). More generally, we need to analyze the activity in the brains of each 
monkey/ape/human when the two interact. Here we address this concern in the realm of computational neuroscience by advocating dyadic brain modeling (Figure 3) which focuses on what happens in the brains of two interacting agents, a dyad, where the actions of one influence the actions of the other, with both brains changing in the process. Luc Steels (e.g., Beuls \& Steels, 2013) has used simulation of embodied agents in evolutionary games in a fashion relevant to studies of (cultural) language evolution (and recall the semiotic cycle of Figure 1). Our innovation here is to provide the agents with "brains" based on prior work in brain modeling. The current implementation (Section 2.2) is simplified, but provides proof of concept for future work which can increasingly address data on the role of neural systems in primate social interaction and do so in an evolutionary context.

\subsection{Computational Comparative Neuroprimatology}

In developing, a Comparative Neuroprimatology, we seek to link behavior (in both praxis and communication) to relevant brain mechanisms, seeking an integrative understanding of the similarities and differences between monkeys, apes and humans. In the present effort, we have three particular foci:

i) Monkeys: Here we have neurophysiological details on activity of single neurons and circuits linked to detailed neuroanatomy. For the present article the key data are related to the role of vision in the generation and recognition of manual actions, and auditory processing of sounds linked to such actions as well as to monkey vocalizations.

ii) Apes: Here we have a range of data on praxic and communicative behavior from studies of captive apes as well as field studies in the wild. In this review, we will focus on the relation of certain praxic actions to communicative manual gestures. However, ape neuroscience data are limited to anatomical studies and to brain imaging addressing connectivity in the anesthetized (i.e., non-behaving) chimpanzees.

iii) Data on the human brain come primarily from neuropsychology (effects of brain damage due to lesions), the study of brain diseases (not only changes in behavior and cognition due to the disease itself, but also the effects of drugs on such changes), as well as varied brain imaging techniques, including ERPs (with relatively precise timing but poor localization) and fMRI (with poor timing but relatively precise localization - but at far lower resolution than single cell recording). Neurolinguistics is the attempt to relate such data to language. As noted earlier, the subject of neurolinguistics per se is tangential to the scope of this article, though it 
is my hope that the approaches reviewed in Section 4 will support further developments in neurolinguistic theory. Arbib (2015) offers a promissory note, while D. Kemmerer (2015) offers a comprehensive review of the state of the art.

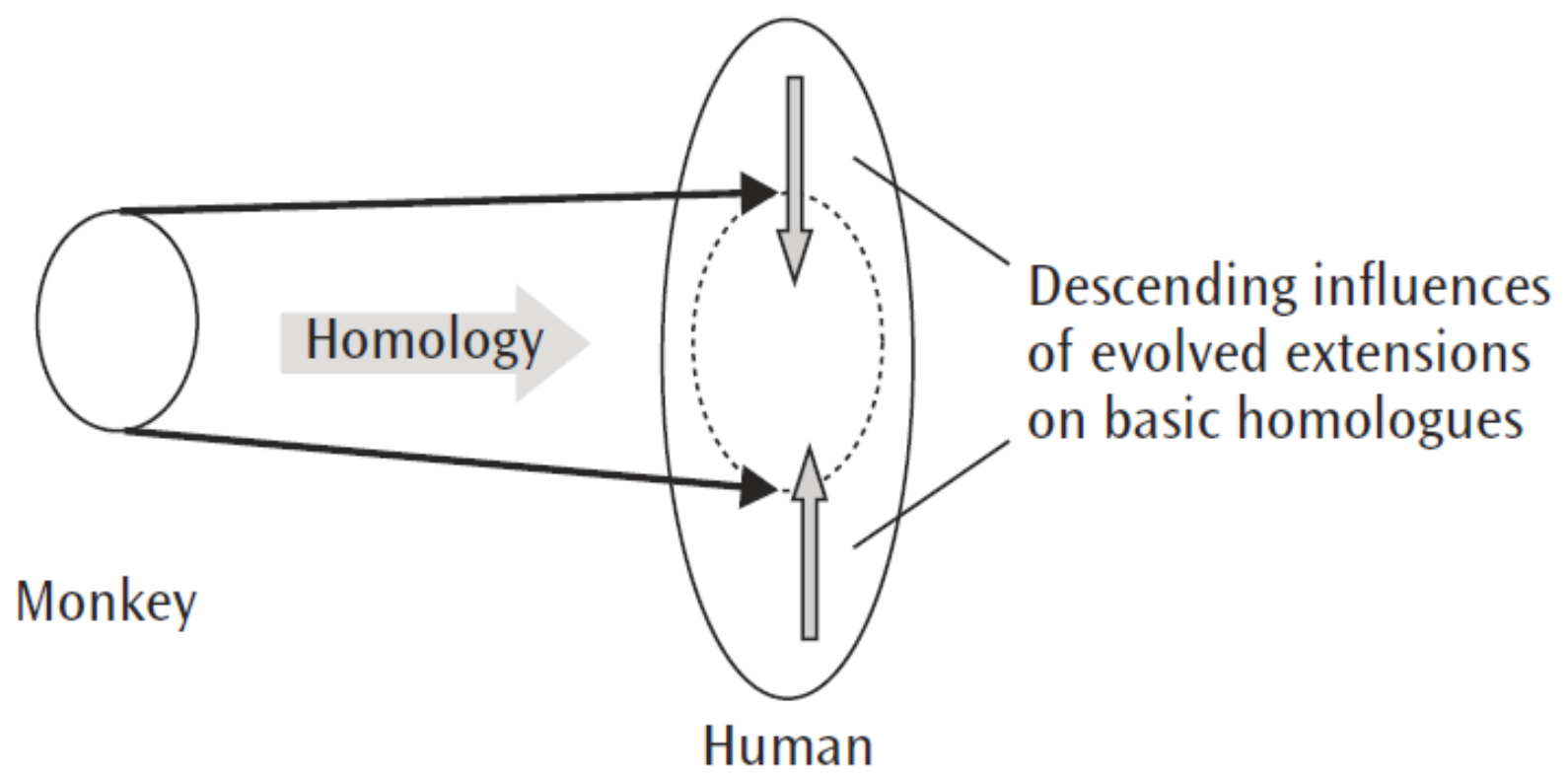

Figure 4. A strategy for modeling the human brain: Use known circuitry in the macaque brain to suggest details of circuitry in homologous regions of the human brain; extend these models using hypotheses on brain evolution; then test the result against or by lesion data, or human brain imaging studies by the use of synthetic brain imaging.

Computational Comparative Neuroprimatology is the attempt to knot together these very diverse datasets by use of computational models of neural (and schema) models. In many cases such as the reach to grasp and the control of eye movements - we may assume that the underlying circuitry as charted in the monkey are relevant to filling in the details obtained from brain imaging and other human studies. Synthetic Brain Imaging (SBI) offers algorithms for averaging over the synaptic activity revealed by simulations of models of neural circuitry to predict region-by-region activity of the kind measured by brain imaging studies. In particular, then, SBI can bridge between neuron-based modeling of animal brains and studies of the human brain for which scant information about the underlying circuitry is available (Arbib, Bischoff, Fagg, \& Grafton, 1995; Arbib, Fagg, \& Grafton, 2002; Tagamets \& Horwitz, 1998, 2000). The methodology is to develop neural network models of human brain mechanisms for which one believes the relevant circuitry is similar to that revealed by animal (e.g., monkey) single cell 
neurophysiology and then process simulation results at the neural network level to infer predictions that can be tested against human fMRI or other non-invasive measures. One can also apply variations of this methodology to schema networks. Related strategies also apply to the development of Synthetic ERPs to allow further testing against human data (Barrès, Simons, \& Arbib, 2013). Figure 6 illustrates an extension of the approach for mechanisms - such as those subserving language - for which nonhuman neural circuitry does not suffice: We use hypotheses about evolution of brain mechanisms to suggest how macaque circuitry is modified and expanded upon in the architecture of the human brain, then use the resultant model to make predictions for human brain imaging or for lesions. In Section 2.2 we will extend this methodology to the comparison of macaque and chimpanzee brains with respect to communicative hand gestures.

Finally, the standard reminder: Humans did not evolves from chimpanzees and chimpanzees did not evolve from macaques. When we turn to evolution, we seek to extend a comparative neuroprimatology based on data concerning extant species by making hypotheses about LCA-m (the last common ancestor of macaque, chimpanzee and human) and LCA-c (the last common ancestor of chimpanzee and human) as a basis for exploring hypotheses about the evolutionary pathway LCA-m $\rightarrow$ LCA-c $\rightarrow$ Homo sapiens.

\subsection{Protolanguage versus Language}

Before going further, we need to clearly distinguish between protolanguage and true language.

A language is characterized by a large (and open-ended) stock of words (the lexicon) and a rich grammar which supports a compositional semantics - i.e., knowing the meanings of words, we can combine them using the grammar not only to build phrases and sentences that express novel meanings, but to also to do so with a fair expectation that these meanings will be understood (the parity principle).

A protolanguage here (as distinct from the usage of the term in historical linguistics) is a system of communication of the kind we hypothesize our distant ancestors had before true languages emerged - a protolanguage rests on "protowords" whose meaning can be established in a community by conventionalization (and in this sense is open-ended), but which has little or no grammar (Arbib \& Bickerton, 2010).

A crucial debate in the study of language evolution concerns whether 
(i) the first Homo sapiens already had language in its full sense, perhaps even with brain mechanisms supporting a Universal Grammar (perhaps along the lines of the Principles and Parameters theory of syntax; see, e.g., Lightfoot (2006) and my critique Arbib (2007)), or

(ii) that the early Homo sapiens brain was language-ready in the sense that such a brain would have been able to acquire language had a language-rich culture existed, but did not yet have language.

MSH adopts the second stance (see Arbib, 2012 for supporting arguments), arguing that the first Homo sapiens had protolanguage but not language. In this review, Section 2 of the MSH account of how biological and cultural evolution together yielded the language-ready brain; Section 3 then sketches how cultural evolution came to endow humans with language. (To put this in perspective, no one doubts that the emergence of agriculture and cities in the last 10 millennia were the results of cultural rather than biological evolution. What about language?) We start, then, with seven properties of "language-readiness" that I see as supported by brain mechanisms that evolved during the emergence of protolanguage but prior to the emergence of language (Arbib, 2012, Chapter 6):

1. Complex Action Recognition and Complex Imitation: This involves an ability for both perceptual and motor chunking supporting:

- complex action analysis, the ability to recognize another's performance as resembling an assemblage of familiar actions,

- the actual imitation, grounded in complex action recognition, to repeat the assembled actions, and, more subtly, the recognition of another's performance as resembling an assemblage of familiar actions as a basis for the imitator to further attend to how novel actions differ from the ones they resemble, thus facilitating the ability to perform these variant actions in due course.

Note that this definition refers to skills in general without necessitating (or denying) any linkage to language.

2. Intended Communication. This is distinguished from, e.g., an observer noting the availability of food because you are eating, even though you have no intention of telling others about the food.

3. Symbolization. In this case, a community adopts a particular symbol to convey a certain range of meaning - in modern society, this could be a word of a spoken language, a sign of a 
signed language, a gesture such as waving goodbye, or a STOP sign on the roadside. However, innate calls such as monkey alarm calls would not be considered as symbols. MSH sees pantomime as a form of intended communication that provides a stepping stone to symbols (the protosigns of Section 2.3).

4. Parity: What counts for the producer of one or more symbols must count, frequently, as approximately the same for the receiver of those symbols. The parity principle for communicative actions extends the role of the mirror neurons for grasping and other actions, "lifting" complex imitation from praxis to communication.

5. From Hierarchical Structuring to Temporal Ordering: Animals can perceive the hierarchical structure of scenes to determine what actions to execute and when to execute them to achieve their goals.

6. Beyond the Here-and-Now 1: The ability to recall past events or imagine future ones.

7. Paedomorphy and Sociality: A prolonged period of infant dependency, especially pronounced in humans, combines with the willingness of adults to act as caregivers and the consequent development of social structures to provide the conditions for complex social learning.

The development of MSH was supported by data consistent with the view that the mechanisms which underlie these seven properties were the result of biological evolution (though certainly in conjunction with "cultural evolution" constructing ever more complex niches for social interaction) and are supported by the genetic encoding of brain and body and the consequent space of possible social interactions.

However, and perhaps more controversially, MSH further claims that no changes in the genome were required for cultural evolution to yield the following four properties that distinguish language from protolanguage and language-readiness (though modern Homo sapiens may have these skills in larger measure than early Homo not only as a result of cultural evolution but also due to some measure of Baldwinian evolution (Baldwin, 1896; Waddington, 1953) that favored offspring with greater skill in exploiting these skills):

8. Symbolization \& Compositionality: The symbols become words in the modern sense, interchangeable and composable in the expression of meaning. 
9. Syntax, Semantics \& Recursion: The matching of syntactic to semantic structures grows in complexity, with the nesting of substructures making some form of recursion inevitable.

10. Beyond the Here-and-Now 2: Verb tenses or other language-specific tools express the ability to recall past events or imagine future ones.

11. Learnability: To qualify as a human language, much of the syntax and semantics of a human language must be learnable by most human children.

In short, MSH claims that the "language-ready brain" of the first Homo sapiens supported basic forms of gestural and vocal communication (protosign and protospeech, see Section 2.3) but not the rich syntax and compositional semantics and accompanying conceptual structures that underlie modern human languages.

\subsection{Introducing Construction Grammar}

A crucial issue in probing the mechanisms that implement language use is how to characterize grammar - and thus language - in a manner that supports modeling both the production and comprehension of utterances. As is well known, many approaches to grammar are "competence" theories; but for neurolinguistics we need approaches to grammar that focus on "performance." Moreover, when we listen to a sentence we are not, usually, concerned with an explicit extraction of its syntactic form so much as an understanding of its meaning. This latter point suggests the relevance of approaches to grammar which integrate, rather than dichotomize, the processing of form and meaning. Here I briefly introduce the general notion of construction grammar as an approach to the integration of form and meaning, and note that it is a "broad church" that contains diverse specific models of grammar but, of most importance to us, includes performance-based models of grammatical processing.

The meaning of idiomatic expressions like kick the bucket or take the bull by the horns cannot be inferred from word meanings via compositional semantics. This suggests that the meaning of idioms must be stored in each speaker's mind. Fillmore, Kay, and O'Connor (1988) took a radical step: Instead of adding idioms to a generative model which separates the lexicon from a syntax considered as a highly general and abstract set of rules for combining words, they argued that the tools they used in analyzing idioms could form the basis for Construction Grammar (CG; Croft, 2001; Goldberg, 1995) as a new model of grammatical organization. CG comes in varied forms, but all blur the division between grammar and lexicon. What is common to these 
various efforts is that the grammar is based not on a small set of purely syntactic rules but instead on constructions which, like items in the lexicon, combine syntactic, semantic and even in some cases phonological information. (A better terminology would call the compositional tools constructors and reserve the term constructions for what, such as phrases and sentences, applications of the constructors do indeed construct.)

In the framework of $\mathrm{CG}, \mathrm{He}$ kicked the bucket has two parsings. One yields an instance of the general formula $H e X^{\prime} d$ the $Y$ whose overall meaning varies with the meanings of $X$ and $Y$. The other employs a construction whose meaning is "He died." As Hurford (2011, Chapter 4) notes, there is evidence that on hearing an idiom, both the overall meaning (e.g. die) and the meanings of the individual parts (e.g., kick and bucket) can be primed, suggesting redundant storage. This fits with our general view of competition and cooperation of schemas in that the initial activation of constructions for both parsings can have a priming effect even though just one eventually wins the competition determining our understanding of the sentence.

Large-scale syntactic categories like noun versus verb may often prove too coarse in specifying which words can fill which slot of a specific construction. For example, the constraints on $\mathrm{Y}$ and $\mathrm{Z}$ in the Goal-object construction $W X^{\prime} d Y$ with $\mathrm{Z}$ as in

Jenny filled the glass with water

*Jenny poured the glass with water

and the Theme-object construction $W X^{\prime} d Z$ in $Y$ as in

Jenny poured water in the glass

*Jenny drenched water in the glass

(* for ungrammatical) cannot be accounted for simply in terms of such general syntactic categories. Indeed fill/drench/soak/saturate/infuse work with the Goal-object and not with the Theme-object construction and vice versa for pour/drip/dribble/spill. Such subtleties support the use of constructions to represent grammatical knowledge, abandoning the duality between lexicon and grammar as well as between syntactic and semantic components.

Here I simply want to note that CG comes in many flavors and list four efforts under the general banner of CG. Each provides ingredients that we need, but each is incomplete:

1. The dynamic CG developed by Peter Dominey and his colleagues (Dominey \& Inui, 2009) has sought to understand how brain models can simulate the learning and use of the formmeaning mappings in constructions. They anchor modeling of the language system on 
previous modeling of the macaque oculomotor system which demonstrated the central role played by cortico-striatal connections in sequence processing (Dominey, Arbib, \& Joseph, 1995).

2. Embodied $C G$ is rooted in a specific version of motor schemas called $\mathrm{X}$-schemas that includes specification of what comes before and after the action and is within the "embodied cognition" tradition that views meaning of a sentence as extracted by mental simulation of the event which a sentence represents, with the range of possible meanings extended via metaphor (Feldman \& Narayanan, 2004; Narayanan, 1997).

3. Fluid $C G$ has been developed in the context of interacting robots sharing visual access to a given environment as a basis for, e.g., converging on a shared lexicon - and, indeed, this work defined the semiotic cycle of Figure 2. It uses evolutionary games between embodied agents as the basis for studying the formation and dynamics of shared meaning (Beuls \& Steels, 2013). This approach is now being advanced as a framework for the formal study of historical linguistics (cultural evolution).

4. Template $C G$ (to be further developed in Section 4.3) is anchored by perceptual (visual) schemas, assessing how the core of language processing may be captured by analysis of the production and comprehension of descriptions of visual scenes .

The reviewer of the first draft stated "There is no reason to assume that (Template) CG is any better suited (or any less well suited) to characterizing language in the brain than any other individual theory of grammar." In one sense the point is well-taken. I do not (cannot!) claim that TCG is "the answer." Rather, my point is that it links language to other brain systems in performance (production and perception) in a way that most other theories fail to do. As Warren McCulloch would say: "Don't bite the end of my finger; look where I am pointing." So Section 4.3 will present TCG as a case study in seeking to develop new grammatical theories in the context of neurolinguistics and with the added bonus of linking to the visual world paradigm in which psycholinguists study eye movements of subjects assessing a sentence related to a visual scene placed before them. This will follow Section 4.2's review of the (non-CG) auditory-based model of Bornkessel-Schlesewsky and Schlesewsky (2013). Each of the four CG frameworks listed above has clear strengths, but only Dynamic CG offers an extended attempt to relate its processes to neural circuitry, and it is limited in the subtlety of its constructions. Each covers only a portion of the mechanisms whose evolution we need to understand if we are to develop an 
approach to language evolution that can make rich contact with studies of the interactions within and between brains. We will learn from each of them in future research.

\section{The Mirror System Hypothesis 1: Evolution of the Language-Ready}

\section{Brain}

The mirror system hypothesis (MSH) argues that a language-ready brain resulted from the evolution of a progression of linkages of mirror systems with brain regions "beyond the mirror." It should be added that brain, body and social structure co-evolved. New social practices endowed certain changes in brain and body with a selective advantage that was not there before; but then certain social practices became newly adaptive (and this may include development and use of new tools). Though a full account must stress gene-culture co-evolution, the key here is the phenotype which results from the development of the individual made possible by brain and body as well as the physical and cultural niche in which development occurs - thus the importance of considering niche construction (Iriki \& Taoka, 2012; Odling-Smee, Laland, \& Feldman, 2003).

The mirror system hypothesis offers an evolutionary framework for the following data:

- Monkeys, apes and humans all have great manual dexterity.

- Macaque mirror neurons respond only to observation of transitive manual actions in the sense of manual gestures that are directed to an actual object.

- Human imitation involves the mirror system ... but macaques don't imitate, while apes do though in a more limited way than humans.

- Monkeys and apes have genetically constrained call systems and no capacity for vocal learning.

- Apes can communicate using novel manual gestures which are intransitive (i.e., not directed at an object), though only to a limited extent.

- Humans have equal facility for language in the manual and vocal domains - signed and spoken languages, respectively (and speakers gesture with their hands and faces as they talk).

Section 2 reviews how MSH charts the path from last common ancestor of macaque and human (LCA-m) via the last common ancestor of chimpanzee and human (LCA-c) to Homo sapiens. We claim that early Homo sapiens had protolanguage but not language, and so Section 3 
will chart how cultural evolution may have supported the transition from humans with a language-ready brain but no language to human societies with language.

Section 2.1 reviews models of neural mechanism, based on neurophysiological data on macaque brains, for execution of manual actions (with special attention to the dorsal and ventral streams for vision), action recognition (suggesting how mirror neurons emerge through learning) and opportunistic scheduling (showing a role for mirror neurons in improving one's own behavior). We hypothesize that these were all brain mechanisms of LCA-m relevant to MSH.

Section 2.2 builds on the models f Section 2.1, introducing dynamic brain modeling to show how apes could acquire gesture through ontogenetic ritualization, whereby a pattern of behavior by one actor becomes reduced to a short gesture that achieves the same distal goal in terms of the response of the other. We hypothesize that an increased ability to use proprioception in controlling actions was one of the key transitions from LCA-m to LCA-c.

Section 2.3 then recapitulates the changes supported by biological evolution from LCA-c to early Homo sapiens. We see pantomime as marking a crucial new capability, creating the ability to freely communicate about a large range of meanings, and thus providing the beginning of an open semantics. However, pantomime can be both laborious and highly ambiguous, and so the next step is the conventionalization of pantomimes and the addition of otherwise arbitrary gestures to yield a system (protosign) that provided economical and less ambiguous symbols, with an open semantics but little or nothing in the way of a grammar. We then claim that mechanisms for flexible vocal control and learning only became adaptive once protosign had revealed the adaptive advantage of open-ended communication using arbitrary gestures. We observe that the claim that the path to speech is indirect is controversial and argue for its plausibility, with protosign and protospeech evolving together (both biologically and culturally) in an expanding spiral to yield a language-ready brain and early humans with flexible protolanguages.

\subsection{Modeling Macaque Brains and Hypothesizing the Nature of LCA-m}

\section{From Macaque Grasping to Mirror Neurons}

Section 1.2 discussed dorsal and ventral streams in vision and audition and, in particular, discussed data linking vision to manual actions which supported the view

- Dorsal stream: Parietal affordances $\rightarrow$ motor parameters for preshape 
- Ventral stream: IT perception of object attributes $\rightarrow$ pantomime or verbally describe these attributes.

Then in Section 1.3, our analysis of the VISIONS schema-theoretic model of interpretation of visual scenes led us to expand our view of the ventral stream to include visual analysis of a scene, not just object perception. Such considerations on the human brain may serve to frame a very brief review of the FARS and MNS models based on data on the interactions between parietal and frontal cortex in the macaque monkey that are implicated in the visual control of manual behavior. In particular, the FARS model may be understood in part as neurobiological elaboration of the schema-theoretic model of reaching to grasp (Section 1.2).

A subarea of macaque parietal cortex named the anterior intraparietal cortex (AIP) contained neurons responsive to visual properties of objects - but visual properties that indicated "affordances" for grasping, rather than the particular category of the object (e.g., cube versus sphere) which is encoded in IT.

Similarly, cells whose activity correlated with the macaque's execution of a particular type of grasp were found in a region of premotor cortex called F5. Of particular relevance for the present review was the discovery of mirror neurons in macaque F5 - each such neuron is active both when the monkey executes a specific set of manual actions (e.g., precision pinches but not power grasps) and when the monkey observes manual actions more or less similar to the actions in that set.

This background should make clear the features of the FARS model of parietal-premotor interactions in primate control of grasping (Fagg \& Arbib, 1998) shown in Figure 5, and the MNS model (Oztop \& Arbib, 2002) of how mirror neurons may be formed by learning based on visual observation of one's own actions shown in Figure 6. Although we do not diagram it here, the MNS2 model (Bonaiuto, Rosta, \& Arbib, 2007) extends the MNS model to explain audiovisual mirror neurons (which, for actions with distinctive sounds [like breaking a peanut] respond whether the monkey sees or hears the action, or both) and the fact that mirror neurons for grasping will not respond to a pantomimed grasp (no associated object) yet will respond to grasp of a recently visible object if the end of the grasp is obscured by a screen. These will be the basis for the chimpanzee model offered in Section 2.2. In particular, we stress that, when presented with the view of an ongoing action, the MNS model learns to strengthen recognition neurons earlier and earlier in the trajectory. 


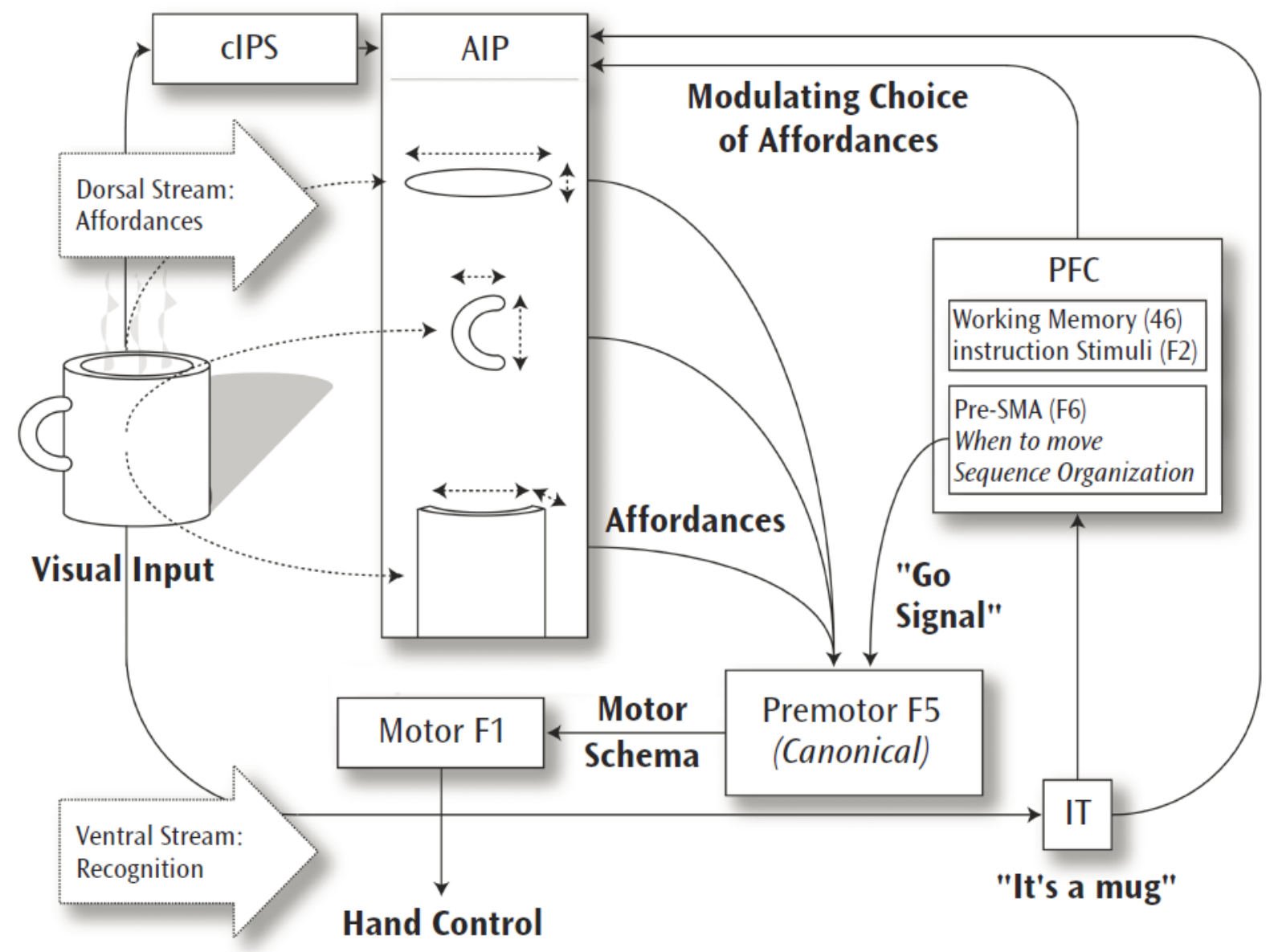

Figure 5. The dorsal stream part of the FARS model takes input from the primary visual cortex and processes it via, especially, area AIP of parietal cortex. The idea is that AIP does not "know" the identity of the object, but can only extract affordances (opportunities for grasping the object considered as an unidentified solid), indicated here by portions of the object which are particularly graspable. The FARS model further integrates the dorsal stream with the ventral stream via inferotemporal cortex (IT). Prefrontal cortex (PFC) uses IT's identification of the object, in concert with task analysis and working memory, to help AIP select the appropriate affordance from its "menu." Given this affordance, F5 can select an appropriate motor schema and instruct primary motor cortex to forward its execution. (The diagram does not specify the pattern of connectivity between subregions of PFC. Further details on this appear in the 1998 paper.) 


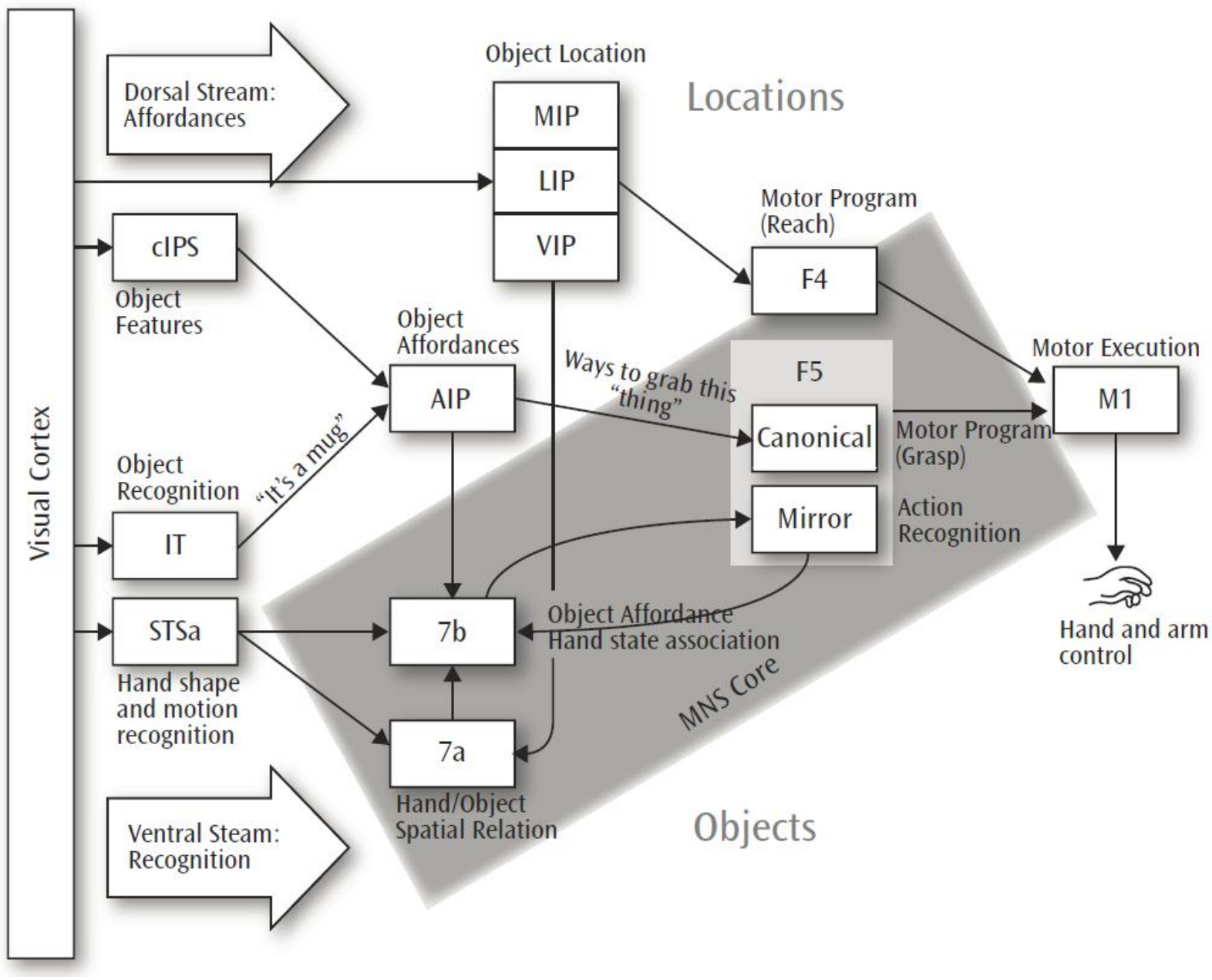

Figure 6. A schematic view of the Mirror Neuron System (MNS) model of the mirror system for grasping. Visual input is processed in different streams to extract the affordances of an object and the shape and movement of a hand. The model describes mechanisms whereby, using the "grasp code" of the canonical neurons of F5 as training signal, particular mirror neurons come to recognize affordance-centered trajectories of the hand that correspond to specific types of grasp. Such a trajectory can thus equally well activate appropriate mirror neurons when the trajectory involves the hand of the self or the hand of another. Note, however, that the model does not address how attention comes to be directed to a particular hand and a particular object. (From Arbib \& Mundhenk, 2005; adapted from the original system figure of Oztop \& Arbib, 2002) Refinements in the MNS2 model (Bonaiuto et al., 2007) explained data on audiovisual mirror neurons and on mirror neurons that could recognize a grasp even if the object had been recently obscured from view.

\section{More Modeling of the Macaque Brain: Augmented Competitive Queuing}

We here introduce a new model, the Augmented Competitive Queuing model (ACQ, Bonaiuto \& Arbib, 2010) which addresses the flexible scheduling of actions to achieve some 
overall goal. Crucially, and unlike most of the mirror neuron literature, this model emphasizes the utility of a mirror system in recognizing one's own action. But first we consider two key distinctions which compare and contrast ACQ with FARS and MNS:

Proximal versus Distal Goals: All the actions in a behavior may be steps towards a single shared goal, the distal goal, yet each may have a distinct proximal goal that shapes that particular action. In the FARS and MNS models, the focus is on a single goal: grasping an object, based on one of its affordances, or recognizing a basic ongoing action. In ACQ the emphasis shifts to scheduling a sequence of actions, with each linked to its own proximal goal which serves as a subgoal for the distal goal which is the overall goal of the current behavior.

Event-Level versus Trajectory-Level Modeling: In many psychological models, each event or trial or action is considered as an indivisible whole, and emphasis is placed on the stringing together of distinct behavioral events by decision processes. By contrast, trajectory-level modeling analyzes, e.g., the trajectory of a hand movement and the temporal relation of neural firing to that trajectory. For example, the FARS and MNS models are trajectory-level - assessing activity during the execution and recognition of an action - whereas ACQ is event-level, scheduling complete actions under the assumption that other neural systems will manage their trajectories.

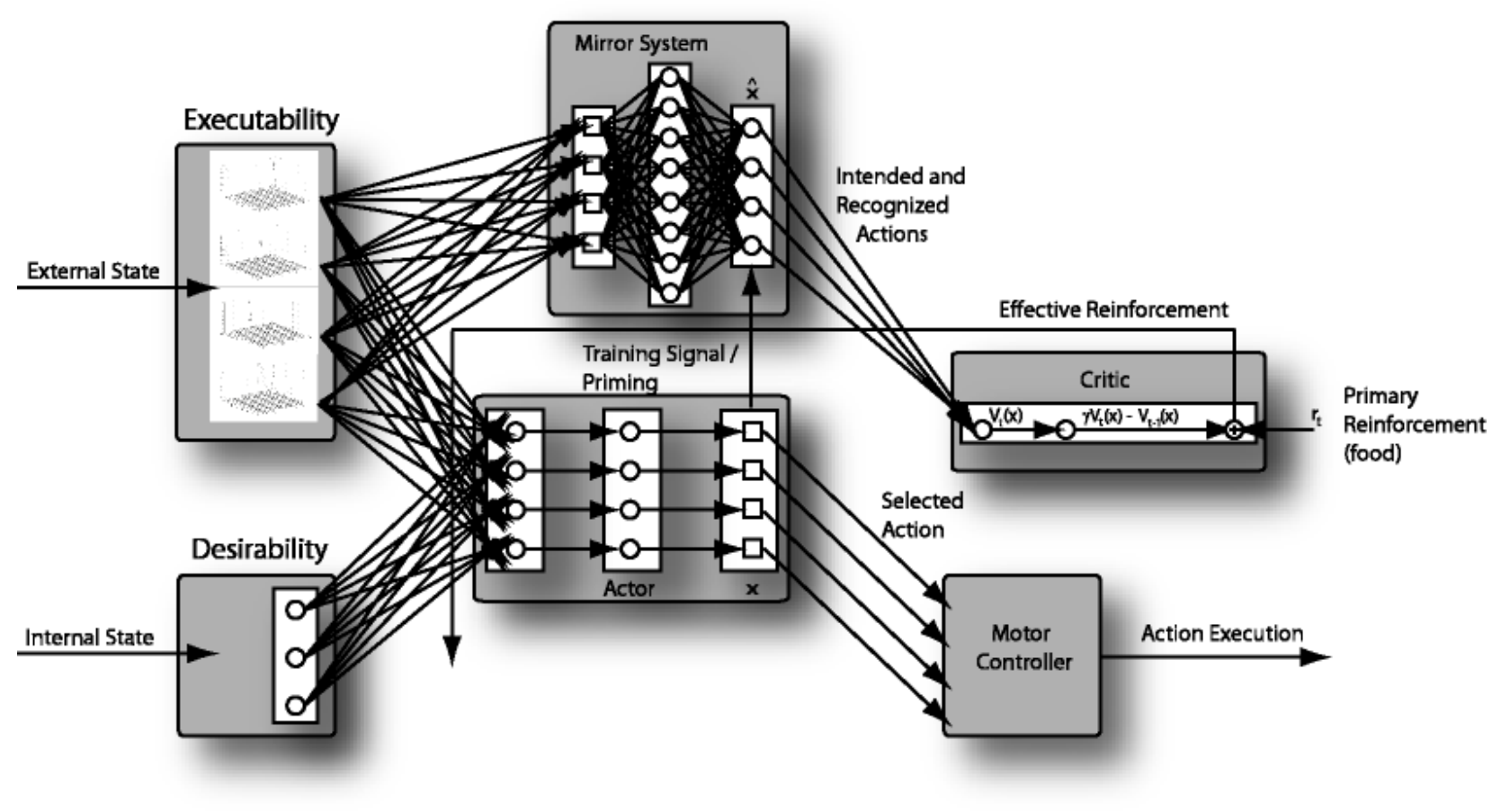


Figure 7. A view of ACQ, the Augmented Competitive Queuing model (Bonaiuto \& Arbib, 2010). The agent works towards its distal goal by choosing the most desirable action that is currently executable. The mirror system monitors self-actions, indicating whether an intended action succeeded or whether the output instead resembled another action, the apparent action. The model includes learning mechanisms for updating estimates of executability of the intended action and desirability of the apparent action.

Augmented competitive queuing (ACQ) is defined in contrast to ordinary competitive queuing (CQ). The idea of competitive queuing (Bullock \& Rhodes, 2003; Houghton \& Hartley, 1995) is that each sequence of actions is coded by setting up a fixed pattern of activity in a neural array, with each neuron coding the "priority" of a particular action. Applying a winner-take-all (WTA) network to this array selects the action with highest priority for execution. What distinguishes a CQ network from a WTA network is "inhibition of return": once an action is executed, the corresponding neuron is inhibited. Thus re-activation of the network yields execution of the action with second highest priority. And so it goes: a spatial coding of priority is converted into a temporal sequence of actions. One of the most famous applications of this idea is in the scheduling of saccades where preprocessing of a visual image yields a map of "salience" of different regions of the image (Didday \& Arbib, 1975; Itti \& Koch, 2000; Koch \& Ullman, 1985); CQ can then direct saccades to these regions in order of their salience.

In ACQ, competition to determine the next action is again based on a priority map, but now this map is updated each time an action is executed. Specifically, separate subsystems in ACQ (Figure 7) assess the executability of actions (e.g., the availability of suitable affordances) and their desirability (an estimate of the expectation that executing that action will lead on to attainment of the distal goal). The model is event-level: the system chooses the action with the highest value of

$$
\text { priority }=\text { executability } * \text { desirability }
$$

and that action is then executed to completion.

Executability and desirability are then updated, and the action with the currently highest priority is executed. This process continues until the distal goal is attained.

Moreover, ACQ contains a mirror system which, when monitoring self-actions, indicates whether an intended action proceeded as expected or whether the output resembled another action, the apparent action. In the current implementation of ACQ (Bonaiuto \& Arbib, 2010) the mirror system is simplified, but to understand the issue here, consider in a little more detail how MNS works (see Figure 6). During training, canonical neurons controlling the monkey's grasp 
are posited to activate, via corollary discharge, a set of potential mirror neurons. This "canonical code" for a grasp serves as the training signal for the latter neurons to learn to recognize the corresponding hand-state trajectories (features coding the movement of the hand and its preshape relative to the selected object affordance). Eventually, the synapses formed under this training become powerful enough that these now-established mirror neurons can fire on observation of an appropriate hand-state trajectory even if it belongs to someone else - thus the mirror neuron property.

Now consider (recalling Figure 6) what happens, according to the MNS model, for selfexecution of an action after the mirror neurons have been trained. The mirror neurons for that action are then activated via two pathways: the canonical pathway brings an efferent copy of the canonical code for the movement while the hand-state trajectory is observed via the visual system (or via the sound of the action driving audiovisual mirror neurons for those actions with distinctive sounds).

The new insight is this: In general, these two pathways will activate the same mirror neurons - but only if the action is successful. If the action fails, then two sets of mirror neurons may be activated: one set for the intended action; and a distinct set for the apparent action if the unsuccessful action happens to resemble an action in the mirror system repertoire. This notion was motivated by analysis of cats that had lost their ability to grasp objects but were able to rapidly develop a new strategy to retrieve food from a glass tube (Alstermark, Lundberg, Norrsell, \& Sybirska, 1981) - see Bonaiuto and Arbib (2010) for the demonstration that the ACQ model with the features we next present could indeed explain this result: a (mirror) system may contribute to rapid reorganization of motor programs in the face of disruption when a known schema can be recognized as "filling the gap" for disrupted schemas. For this reason, Bonaiuto and Arbib titled their paper "What Did I Just Do? A New Role for Mirror Neurons."

The mirror system's evaluation of the end-result of attempting the intended action is used to update the estimate of its executability, downgrading it if the apparent action departs too far from the intended action.

Temporal difference (TD) reinforcement learning (a way to estimate expected reinforcement when actual reinforcement is intermittent, Sutton \& Barto, 1998) is used to update the desirability of the apparently executed action, whether it is the intended action or a different 
apparent action: the desirability of an action is greater the more likely it is to be on a path to attaining the distal goal and the closer it is along the path to the action that actually achieves it.

An extension of ACQ (Arbib \& Bonaiuto, 2012) handles multiple possible distal goals, with the desirability of an action depending on the current selection of distal goal. This then requires modeling the processes setting the current distal goal. Achievement of a distal goal - e.g. eat versus drink - may remove that goal, leading to promotion of another distal goal and consequent resetting of desirability, and so behavior.

\subsection{Modeling Chimpanzee Brains and Hypothesizing the Nature of Evolution from LCA-m}

to LCA-C

We now offer hypotheses on the evolution of capabilities for praxis from LCA-m to LCA-c, suggesting that the greater ability for imitation in present-day apes as compared to monkeys be taken as evidence for a similar expansion from LCA-m to LCA-c. We also take evidence that monkey vocalizations are innate and stereotyped (though open to some sensorimotor tuning) whereas apes have at least as capable a vocal repertoire as monkeys and a somewhat flexible gestural repertoire to support the hypothesis that LCA-c had a capacity, lacking in LCA-m, for learning novel communicative gestures that marked a significant (and semantic) step forward in the evolutionary path that eventually yielded the language-ready brain (Arbib, Liebal, \& Pika, 2008).

\section{Imitation}

We first need to engage in some behavioral comparative primatology contrasting the types of imitation exhibited by monkeys, apes, and humans. Despite the slogan "Monkey see, monkey do," monkeys have, at best, a very limited capacity for imitation (Visalberghi \& Fragaszy, 2001) far overshadowed by what I call simple imitation as exhibited by apes. Byrne and Byrne (1993) found that gorillas learn complex feeding strategies but may take months to do so. Consider eating nettle leaves. Skilled gorillas grasp the stem firmly, strip off leaves, remove petioles bimanually, fold leaves over the thumb, pop the bundle into the mouth, and eat. The challenge for acquiring such skills is compounded because ape mothers seldom if ever correct and instruct their young (Tomasello, 1999) and because the sequence of "atomic actions" varies greatly from trial to trial. Byrne (2003) implicates imitation by behavior parsing, a protracted form of statistical learning whereby certain subgoals (e.g., nettles folded over the thumb) become evident 
from repeated observation as being common to most performances. Having recognized such subgoals, the young ape may acquire the skill over many months trial-and-error to derive action strategies for achieving the subgoals. An open challenge for modeling is to extend ACQ to include the learning of novel actions, not just improved scheduling of actions already in the repertoire.

- By contrast with learning the overall structure of a specific feeding behavior over many, many observations, in many cases of praxis, humans need just a few trials to make sense of a relatively complex behavior if the constituent actions are familiar and the subgoals these actions must achieve are readily discernible, and they can use this perception to repeat the behavior under changing circumstances. As noted earlier, this combination of complex action analysis and actual imitation is called complex imitation. (This extends the definition of Arbib, 2002; to incorporate the goal-directed imitation of Wohlschläger, Gattis, \& Bekkering, 2003.)

Crucially, a mirror system does not provide imitation in itself. A monkey may have mirror neurons related to an action in its repertoire but cannot use observation of a novel action to add that action (movement + goal) to its repertoire (compare previous comment on ACQ). Thus, MSH hypothesizes that evolution extended a monkey-like mirror system in the last common ancestor of human and monkey (LCA-m) into more powerful systems in two stages:

A simple imitation system for grasping, shared with the last common ancestor of human and chimpanzees (LCA-c); and

A complex imitation system for grasping that developed in the hominid line since that ancestor.

The latter process may yield fast comprehension of (much of) the overall structure of the observed behavior. However, the new motor schema may require much practice to yield truly skillful behavior.

The development of computational models of neural mechanisms underlying simple imitation and complex imitation, and an assessment of the extent to which they build upon, or diverge from the MNS models (which must be extended for assessing what the other is doing when the observed behavior is not in one's own repertoire) and the FARS model (for doing a new action oneself once one has acquired it) is a current research challenge. Indeed, relating the difference between simple and complex imitation to differences between chimpanzee and human brains is 
an open research issue. However, readers interested in addressing such challenges might start with the following publications: (Custance, Whiten, \& Bard, 1995; Oztop, Kawato, \& Arbib, 2013; Sauser \& Billard, 2006; Schaal, Ijspeert, \& Billard, 2003).

Complex imitation was an evolutionary step of great advantage for sharing acquired praxic skills. It was thus adaptive for protohumans independent of any implications for communication. However, in modern humans it undergirds the child's ability to acquire language, while complex action analysis is essential for the adult's ability to rapidly comprehend novel compounds of "articulatory gestures."

What evolutionary path led to a brain that could extend complex communication to the communicative domain with sufficient finesse to support language? To start on this path we gather clues about LCA-c from studies of present-day ape communication - which does not extend to language but which combines an innate repertoire of vocalizations with a more flexible repertoire of manual gestures. Some authors (e.g., Hobaiter \& Byrne, 2011) argue that ape gestures are either "species typical" or produced by only a single individual. However, a few group-specific gestures have been observed in ape populations, suggesting a role for social learning (Arbib et al., 2008). Tomasello and Call (1997) proposed ontogenetic ritualization as a means whereby (some) ape gestures could emerge:

1. Individual A performs praxic behavior $\mathrm{X}$ and individual $\mathrm{B}$ consistently reacts by doing $\mathrm{Y}$.

2. Subsequently, B anticipates A's overall performance of $X$ by starting to perform $Y$ before A completes $\mathrm{X}$.

3. Eventually, A anticipates B's anticipation, producing a ritualized form $\mathrm{X}^{\mathrm{R}}$ of $\mathrm{X}$ to elicit Y.

Shortly, we will introduce a model grounded in properties of the mirror system for the brain mechanisms that could support ontogenetic ritualization.

A word of warning, or encouragement, here. My current hypothesis is that it was the linkage of mirror systems to evolving systems beyond the mirror that underwrote the transition to increased ability of imitation manifest by apes and assumed to have been available to LCA-c. However, the remaining stages of MSH do not depend on whether or not mirror neurons play a key role in imitation. The disentangling of their contribution is a current challenge in comparative neuroanatomy of primates, but impeded by the fact that we cannot have single cell recording data for apes or humans (with rare exceptions justified by human neurosurgery). In 
stressing the importance of imitation, MSH has much in common with Merlin Donald's (1991; $1998 ; 1999)$ theory of the evolution of culture and cognition which is based on mimesis and the exploitation of this capability to create mental representations with the critical property of voluntary retrievability.

\section{Grounding a Model of Ape Gestural Development in Macaque Brain Models}

We now focus on ontogenetic ritualization which was, as we have seen, proposed as a means whereby some ape gestures could emerge through repeated interactions between pairs of individuals. Even though the role of this process remains controversial, we argue that using computational neuroscience to hypothesize brain mechanisms that could support ontogenetic ritualization can generate new ideas for primatological as well as neurophysiological and neuroimaging studies, and may reset the debate in fresh ways.

A macaque's recognition of manual actions has only been demonstrated to activate mirror neurons for transitive actions, i.e., actions carried out on an object (e.g., Umilta et al., 2001). Macaques do have mirror neurons associated with transitive ingestive actions and similar orofacial communicative gestures (Ferrari, Gallese, Rizzolatti, \& Fogassi, 2003) - and we have failed to address this in either our MNS modeling or in MSH. Here we focus on the fact that apes are responsive to non-transitive manual gestures, and we may plausibly infer that apes have mirror neurons responsive to many of these gestures. Note, though, that ontogenetic ritualization

yields an ability by one ape to emit a gesture and by another ape to recognize that gesture, but does not posit motor skill for this gesture to the observer. Further changes (e.g., through imitation) would be required to yield mirror neurons in each brain active for both production and perception of the gesture.

The Conceptual Model

For conspecifics, we assume that each brain has the same overall architecture but each agent/brain starts in a different state (in terms both of neural firing and synaptic encoding of skills and memories), and thus the actions and learning processes that unfold will differ between the two agents. We first present the interactions of two apes which support the six graded stages whereby, according to Gasser, Cartmill \& Arbib (2014), a gesture for beckoning arises through ontogenetic ritualization. We then introduce an extended conceptual model to represent the brain of each member of the dyad and then a reduced, computationally implemented version of the model (Arbib, Ganesh, \& Gasser, 2014). 
1) Child (C) reaches out, grabs, and tugs on Mother (M), causing $M$ to move towards $C$ as a response.

C's distal goal is "Bonding" and ACQ-C (the child's version of ACQ) schedules the actions

c1: Reach to grasp M's upper arm, and then

c2: Tug on $\mathrm{M}$ till she moves towards $\mathrm{C}$. This combines a proximal goal of tugging with the distal goal of "Bonding."

Initially, $\mathrm{M}$ has no specific goal. However, once she recognizes C's distal goal, she creates her own distal goal of Bonding with proximal goal "Move close to C" and ACQ-M then schedules

$\mathrm{m} 1$ : Move in the direction of $\mathrm{C}$.

m2: Embrace C.

All four actions are assumed already in the repertoire of $\mathrm{C}$ or $\mathrm{M}$ as body-centered actions. MNS-M recognizes C's distal goal on the basis of the proximal goal of $\mathrm{c} 2$, but this recognition is (in this example) at first based on the haptic input of C's tugging, not on visual recognition. This stage can be seen as the "praxic alternative" to a ritualized gesture, since if later a gestural communicative bout fails, $\mathrm{C}$ can always revert back to this praxic action sequence.

2) $C$ reaches out and grabs $M$, and $M$ moves towards $C$ as soon as $C$ begins to tug.

This stage could proceed over many trials as MNS-M (Mother's action recognition system) comes to recognize c2 more swiftly: With a visual stimulus, the MNS model learns to strengthen recognition neurons earlier and earlier in the trajectory. We now exploit this property during the haptic trajectory induced in $\mathrm{M}$ by c2. As $\mathrm{M}$ recognizes the proximal goal of $\mathrm{c} 2$, and thus C's distal goal, more quickly, she can respond earlier and earlier. Eventually, $\mathrm{C}$ no longer needs to complete $\mathrm{c} 2$. Here we exploit the notion that any action (in this case, C's execution of c2) can be truncated when its proximal or distal goal is achieved.

3) $C$ reaches out and makes contact with $M$, and this is enough to get $M$ to move towards $C$.

Eventually $\mathrm{C}$ need only complete $\mathrm{c} 1$ for $\mathrm{M}$ to respond. ACQ-C continues to schedule $\mathrm{c} 1$, but now c2 is no longer executed because $\mathrm{c} 1$ alone achieves the distal goal. MNS-M then comes to associate c1, rather than c2, with C's distal goal. This stage, like stages 1 and 2, seems not to be a distinction between macaque and chimpanzee. As a further result of Stage 2, the increasingly early termination of the intended $\mathrm{c} 1$, "reach to a position that supports $\mathrm{c} 2$, grasp firmly enough to tug," appears more and more similar to the action $\hat{c} 1$, "reach to touch," and ACQ-C will increase 
the desirability of the latter action so that it comes to replace c2 in C's behavior. And MNS-M will recognize this modified action as also having "Bonding" as its distal goal in this context.

4) $C$ reaches out towards $M$, attempting to make contact, but $M$ responds before contact is made.

In this stage, contact with $\mathrm{M}$ is still C's proximal goal and the action is still transitive. Whereas MNS-M linked tactile feedback to M's responses we can now see robust visually guided adaptive behavior emerge. Seeing M's response, C can now truncate c1 (or ĉ1). However, C's action is still initiated as a movement towards M's arm and so may still be deemed transitive. 5) $C$ reaches out towards $M$, no longer attempting to make contact, yet $M$ still responds by moving towards $C$.

We now postulate that the brains of "chimpanzees" have far stronger pathways bringing proprioceptive input (as distinct from visual, haptic and auditory input) than would be available in the macaque brain.

This means that (in this scenario) as $\mathrm{M}$ responds earlier in recognition of C's goal, $\mathrm{C}$ comes to associate the distal (not the proximal) goal with the proprioceptive state for the truncation of that action part way along the original transitive trajectory. It is not that $\mathrm{C}$ loses the ability to perform action $\mathrm{c} 1$ in appropriate circumstances but rather a new action $\check{c}$ emerges in C's repertoire: performing a reach that has achieving that proprioceptive state (rather than contact with $\mathrm{M}$ ) as its proximal goal - but still with the distal goal (get $\mathrm{M}$ to come closer/bonding) that constitutes the meaning of what is now a novel gesture.

6) C “beckons" towards $M$

The ritualized gesture $\check{c}$ is now part of the action repertoire of $\mathrm{C}$ so that $\mathrm{C}$ can "beckon" to elicit M's response. As in the above stage, $M$ is capable of recognizing his gesture and responding appropriately. Note that apes tend to use visual-only gestures when they have the other's attention. Thus $\mathrm{C}$ might retain the tug strategy when $\mathrm{M}$ is otherwise engaged, but use $\check{c}$ for times when $\mathrm{M}$ is attending visually - learning this gesture has not removed C's original behavior but has refined its context.

Having just postulated that the brains of "chimpanzees" have stronger pathways than is available in the macaque brain to bring proprioceptive input (as distinct from visual, haptic and auditory input) into control of reaching and hand shaping, we note that Hecht et al. (2012) used Diffusion Tensor Imaging to conclude that chimpanzees have "stronger connections" between superior temporal sulcus and inferior parietal cortex (viewed as the posterior mirror system) than 
do monkeys and that this may allow more processing of the finer details of the spatial/kinematic structure of observed actions. How this relates to proprioceptive input requires further analysis. Additional challenges for related research include specifying what exactly is meant by such connectivity changes (and see J. K. Rilling et al., 2008, for changes relevant to language evolution), and how these data affect modeling projects. Do these results implicate new connections between modules, stronger existing connections between modules on a per-species basis, or actual processing differences between and/or within modules?

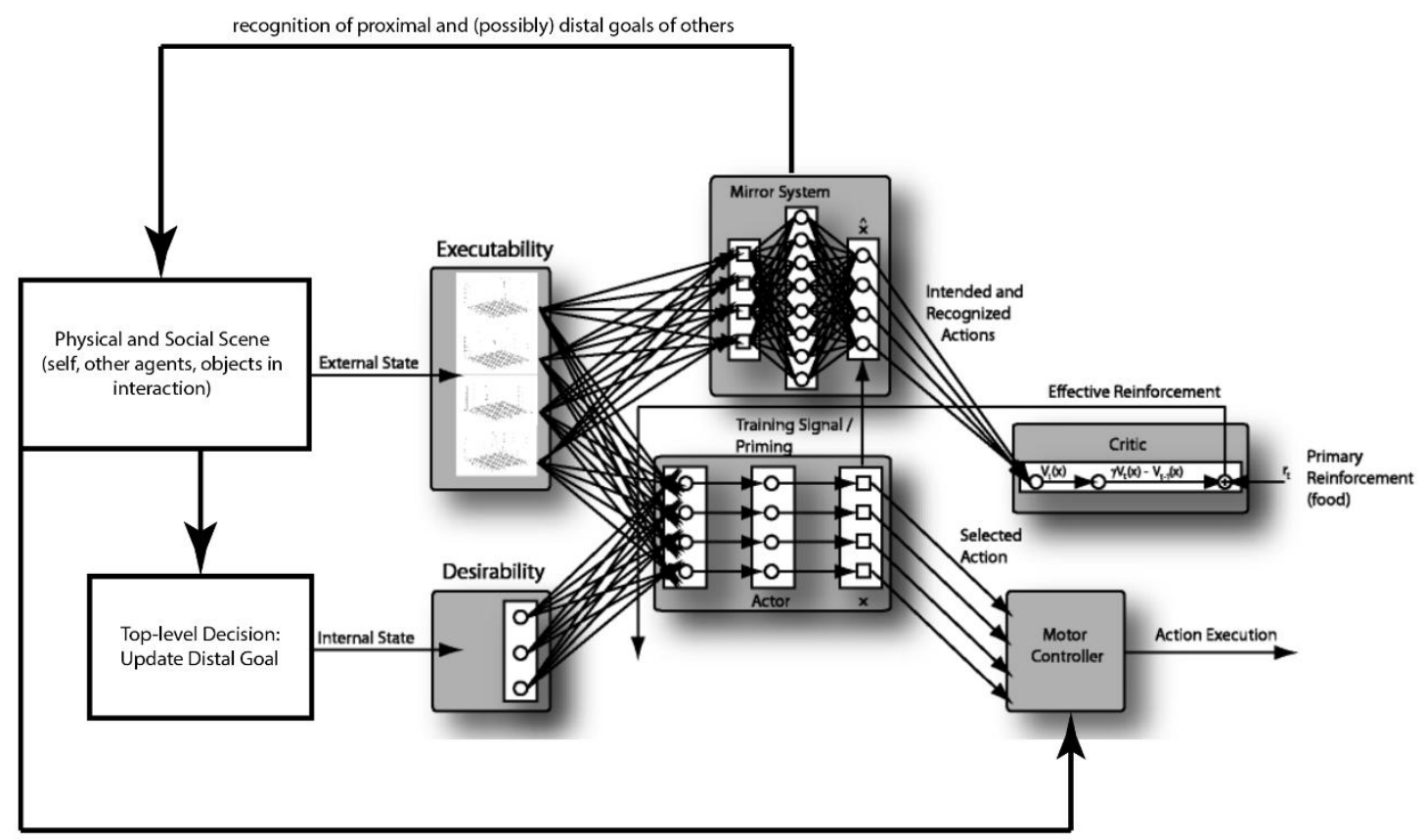

Figure 8. The overall model of the brain of each ape in a dyad, adapting the ACQ model to support ontogenetic ritualization. Updating the current distal goal is an explicit part of the model of each brain in the dyad, and this in turn controls desirability. The mirror system not only plays an ACQ-like role in the analysis of self-actions but will also recognize the actions (movements and goals) of others. Perception of the physical and social scene sets up decisions on the current distal goal as well as providing input to diverse processes. Among the factors not shown here are that modeling is at the trajectory-level, and that proprioception plays a key role in the generation and monitoring of one's own actions.

We now summarize changes required from the ACQ model (Figure 7) to generate our new model of "ape" brain mechanisms shared by $\mathrm{M}$ and $\mathrm{C}$ (Figure 8). However, the requirements below are not beckoning-specific and (i) through (iv) represent abilities of general utility to macaques as well as chimpanzees. 
i) There is a mechanism for social decision making that can use recognition of the distal or proximal goal of another as a basis for possible updating of one's own goals.

ii) Decision making occurs in two stages. A distal goal is set; this goal then sets up the desirability values used by ACQ to schedule a sequence of actions to achieve that goal. (Of course, this does not preclude changing the distal goal as new information becomes available.)

iii) The actions available to the new and extended version of ACQ are all trajectory level (unlike our present ACQ model). Depending on the stage of learning, a mirror system for an action may be sensitive to a goal and more or less sensitive to trajectory.

iv) Each action in the repertoire of the agents of our dyad will be terminated when it is signaled that its proximal goal is reached. Moreover, ACQ can abort a whole behavior once its distal goal is reached.

We see the above additions as an extension of our models of what is common to macaque and chimpanzee. We now turn to a hypothesis on what is different about the chimpanzee:

v) Proprioceptive information becomes increasingly available to augment visual input in mechanisms underlying the learning of novel actions.

\section{The Reduced Model and its Implementation}

We now describe a simulation framework for dyadic brain modeling and then present a simplified model we have implemented. The two model brains are implemented in separate python scripts (Figure 9), employing freely available python packages, including numpy <numpy.org> for mathematical calculations and pybrain <pybrain.org> for neural network algorithms. Each script is linked to Maya animation software through a third python script executed in the Maya python shell. Each brain script can communicate to Maya, via this shell script, through file read-write cycles managed through their separate pipe. Each brain script implements functions from a "brain module" library - for action recognition, decision making, and motor control - to maintain consistency in their implementation. Each script can read relevant joint and effector positions and information about the virtual world in their shared file, and write commands to it for the avatar to execute. Each "brain" uses sensory data on the relation of its body parts to each other and the world, but Maya draws the bodies in a coordinate space appropriate to the view of an external observer. 


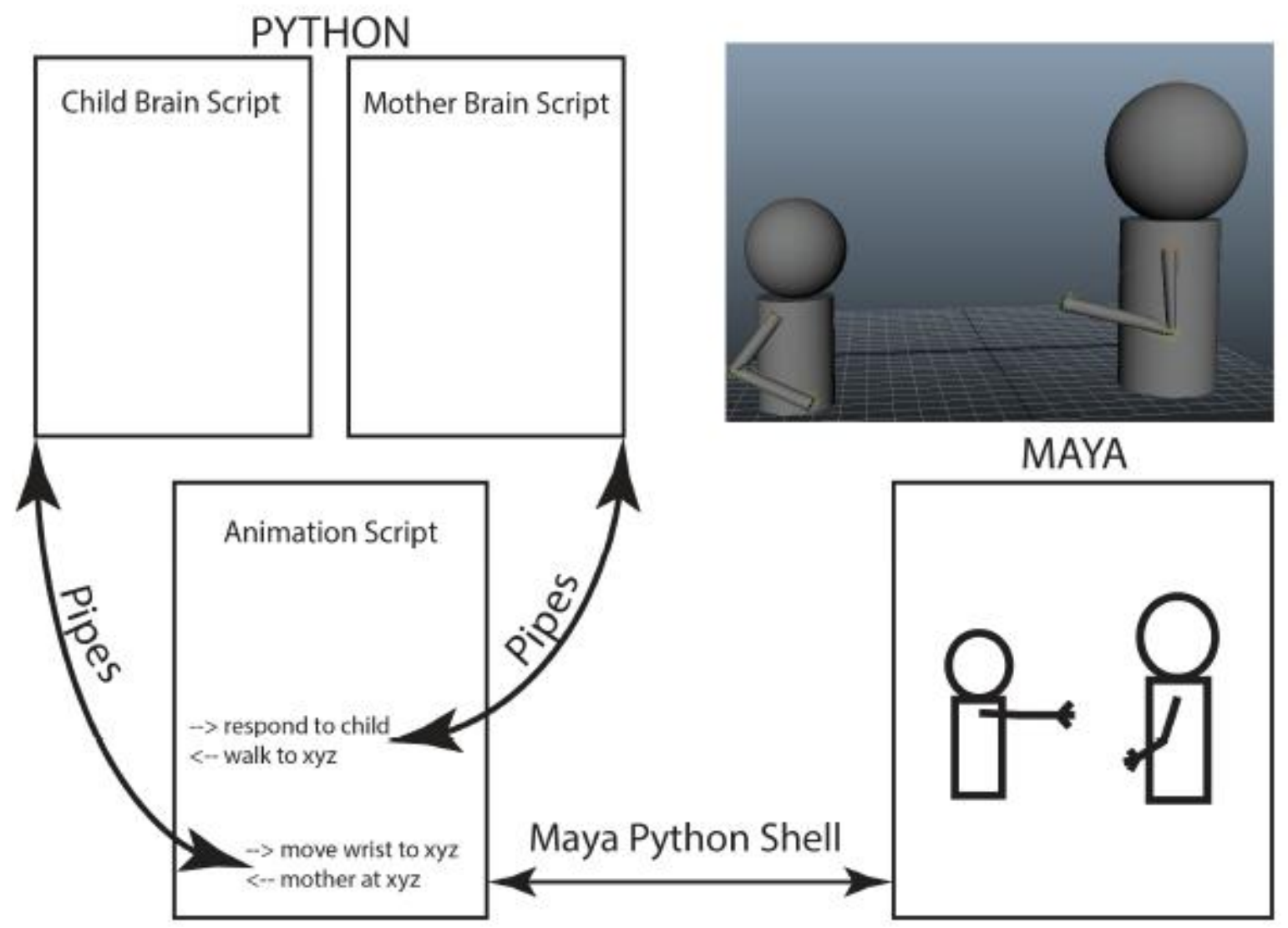

Figure 9. A general computational framework for dyadic brain simulation and visualization of the interaction of two agents. (Clearly, the methodology is not limited to simulating a Mother and Child, but could be adapted to model the neural underpinnings of social interactions of two or more agents.) (Top right) A view of the two avatars $($ small $=$ Child; large $=$ Mother in this example) as displayed by the animation software Maya. In the current simulations, all that changes in these displays is the position of each body in the ground plane, and the angles that define arm postures. (Rest of figure) Simulation overview: The two model brains are implemented as separate python scripts that can communicate only through Maya's animation script.

We now turn to the simplifications of the model of Figure 8 used in the present simulations (Arbib, Ganesh, et al., 2014; readers are referred to this paper for the equations and parameters used in our simulations). The simulations are simplified by the fact that the python script for each agent can read information about the current state of the other agent from the Maya animation script. We thus avoid the need to simulate retinal input to each agent and consequent visual processing to extract state information about the other. However, simulation is still required to infer the action or goal of the other agent from the ongoing trajectory of observed state features plus haptic input. 


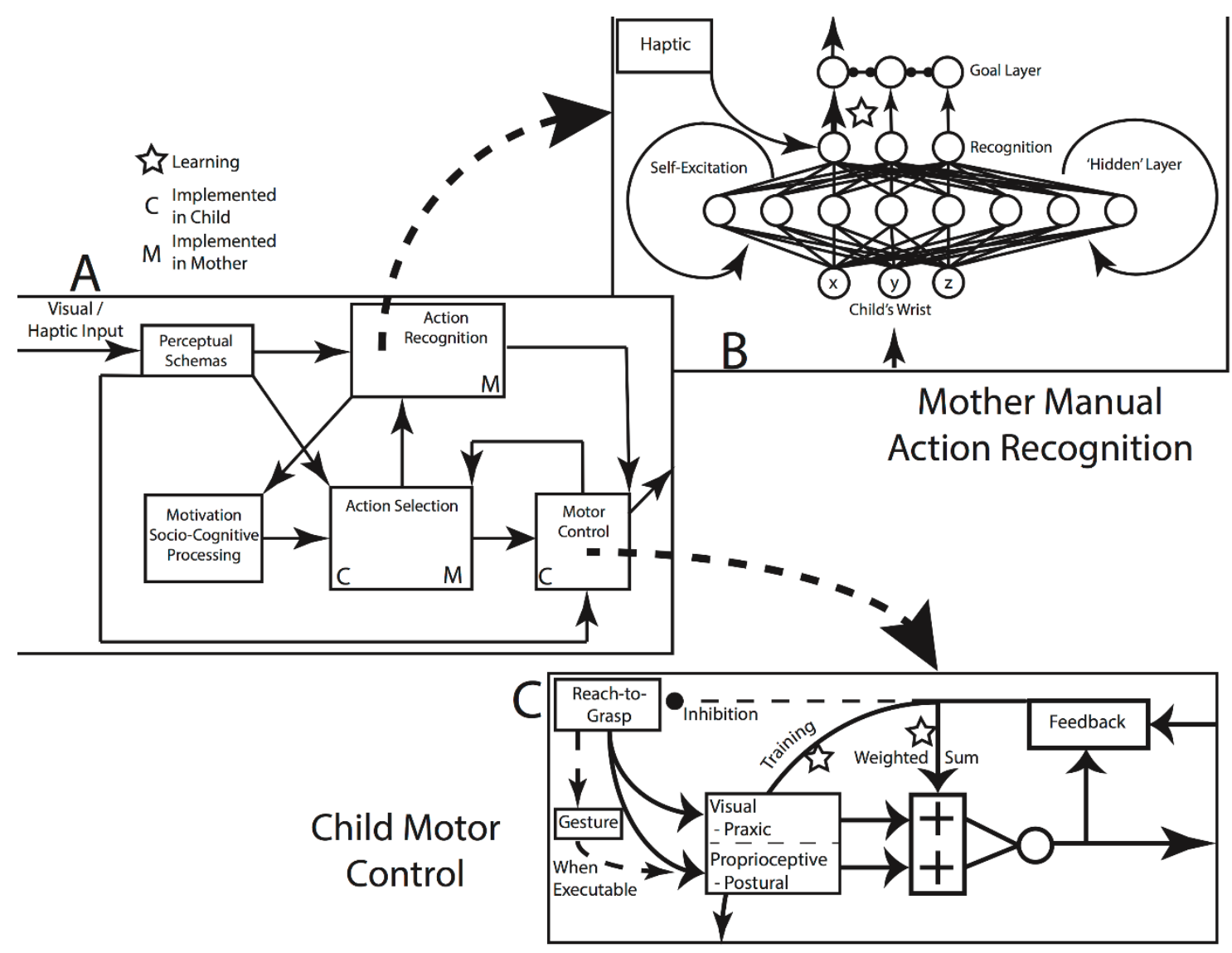

Figure 10. (A) Schematic of the reduced version of the brain architecture for both agents of the dyad. In the simple simulations reported here to demonstrate ontogenetic realization, only some parts are implemented as neural rather than algorithmic models, and differently for Mother and Child. "M" indicates modules implemented "neurally" for Mother, "C" for Child. Other modules are implemented algorithmically. (B) Action recognition for manual actions as implemented for Mother. (C) Motor control as implemented for Child.

The overall architecture of each brain (Figure 10A) was the same, but to reduce computation time, learning in the mirror system was implemented only for Mother, and motor learning for the reach-to-grasp only for Child. Decision-making processes were implemented similarly for both agents, and followed the ACQ paradigm (e.g., combining executability and desirability to yield priority). Our simulations consist of multiple Episodes, each a single explicit case of interaction between the two agents. An Episode may vary from (roughly) 50 to 100 simulation time-steps (though the presumed interaction may last only a few seconds). Visual and haptic sensory inputs to each model represent the output of visual and somatosensory cortical areas, and these inputs may be the result of the others' on-going motor output. 
Action Recognition modules, processing visual and haptic information to recognize self and others' actions, can affect social behavior by linking recognition of others' actions and goals with one's own. Such modules may also provide feedback to learn about the trajectory that achieves a goal. Motivational and Socio-Cognitive processing selects distal goals which yield new action policies. Each agent is initialized in different motivational states: the Child to 'Bond' with the mother (a social goal), and the Mother to remain idle. Following the paradigm of ACQ, these different goal states lead to different behavioral policies for each of Mother and Child: the mother remains idle, while the child executes a sequence of actions to lead to Bonding. However, as a result of learning and recognition processes, these interactions adapt, and the mother's rapid updating of her goal lead to anticipatory responses that achieve the Bonding goal for both.

Figure 10B shows how manual action recognition is implemented for Mother. Action recognition is managed algorithmically for $\mathrm{C}$ in this implementation (meaning we do not simulate a neural network for this process, but instead situate our code to achieve the desired behavior). C's wrist $(\mathrm{x}, \mathrm{y}, \mathrm{z})$ information is input to a neural network with recurrent, selfexcitatory hidden units to maintain temporal information about the inputs. These are converted from 3-D absolute-coordinates in Maya to yield 3-D coordinates relative to C's posture: the wrist trajectory being relative to his starting posture. The recurrent network and its learning mechanism are based on MNS2. Its outputs activate to the degree to which they recognize the time-series data. These units, along with haptic units representing haptic feedback if present (that is, that another has grasped one's arm, for example), then pass activation to goal-layer neurons, via modifiable weights, which compete between each other. If one passes some activation threshold, they switch the internal state of $\mathrm{M}$, so that she shares the Bonding goal of $\mathrm{C}$ and will cooperate towards achieving that goal. More generally, recognition of another's goals may but need not lead to goal-switching in the observer, and new goals may be quite different from the other's, leading not to cooperation, but to competition for example.

M's motor control elements are managed algorithmically, as is walking in C. C's reach-tograsp (Figure 10C) is implemented as an inverse-kinematic controller managing transport of the arm to a target. We model learned target positions in shoulder-centered coordinates, but achieve inverse-kinematic solutions through the animation software. Target data are based on a weighted-sum of visual and proprioceptive data but learning processes may bias each modality's influence. An action will terminate when its proximal goal is reached, and an ongoing behavior 
will terminate when its distal goal is reached (which may involve recognition of the other's behavior).

With this, we turn to some further details of running the Figure 10 model. In each episode, C is initialized with Bonding with $\mathrm{M}$ as his distal goal, while $\mathrm{M}$ is at first passive. This leads to setting C's proximal goal and action. Since the avatars are initialized at some distance from each other, the Child must first walk towards his Mother. When walk is selected, a target position is computed for the agent to walk towards, namely the threshold at which the Child can reach towards the Mother. When the Child reaches this threshold, the "reach-to-grasp" action becomes executable, and since it is more "desirable" in the sense that it is more closely associated with the goal, it is selected for execution. The controller for this action has two sources of sensory data, visual (which can suffice for object-directed actions) and postural (which would suffice for proprioceptively guided action). Each alone can determine a target for the reaching action.

Learning processes adapt the above to quickly generate new patterns of behavior. M's Action Recognition module (Figure 10B) is centered around a recurrent neural network (RNN) modeled after the MNS2 model (Bonaiuto et al., 2007). The RNN receives filtered visual input of C's wrist: the codes must be relative so recognition is possible in different locations. The network is trained through the backpropagation-through-time (BPTT) algorithm (Werbos, 1990) available through pybrain, and is trained on a set of three different reaching actions of $\mathrm{C}$ - the two others being reaching to locations other than M's wrist - each corresponding to a single output unit of the RNN. During simulations, C's Reach will activate a specific output unit to the degree to which its trajectory matches the training data (the first Episode Reach).

C maintains the potential for a full reach-to-grasp-to-pull, but learns over time to (i) pull less, and then (ii) reach less effortfully. He learns at first that $\mathrm{M}$ responds to his pulling, and so pulls her for shorter distances - remaining sensitive to her responses - before learning to only intend to make contact with her wrist. Then, as $\mathrm{C}$ reaches, he learns postural targets he can move to without invoking praxic control mechanisms. In each episode, he updates his postural target as the position at which he terminates his action. Simultaneously, he learns to value this postural target over his visual target. Because it is less effortful and so more desirable, the postural target comes to be selected as the "proximal" goal in service of the distal goal of Bonding. This leads to gesturing behavior that is not constrained by the peri-personal reach threshold: the child can gesture from a greater distance removed from his mother. 


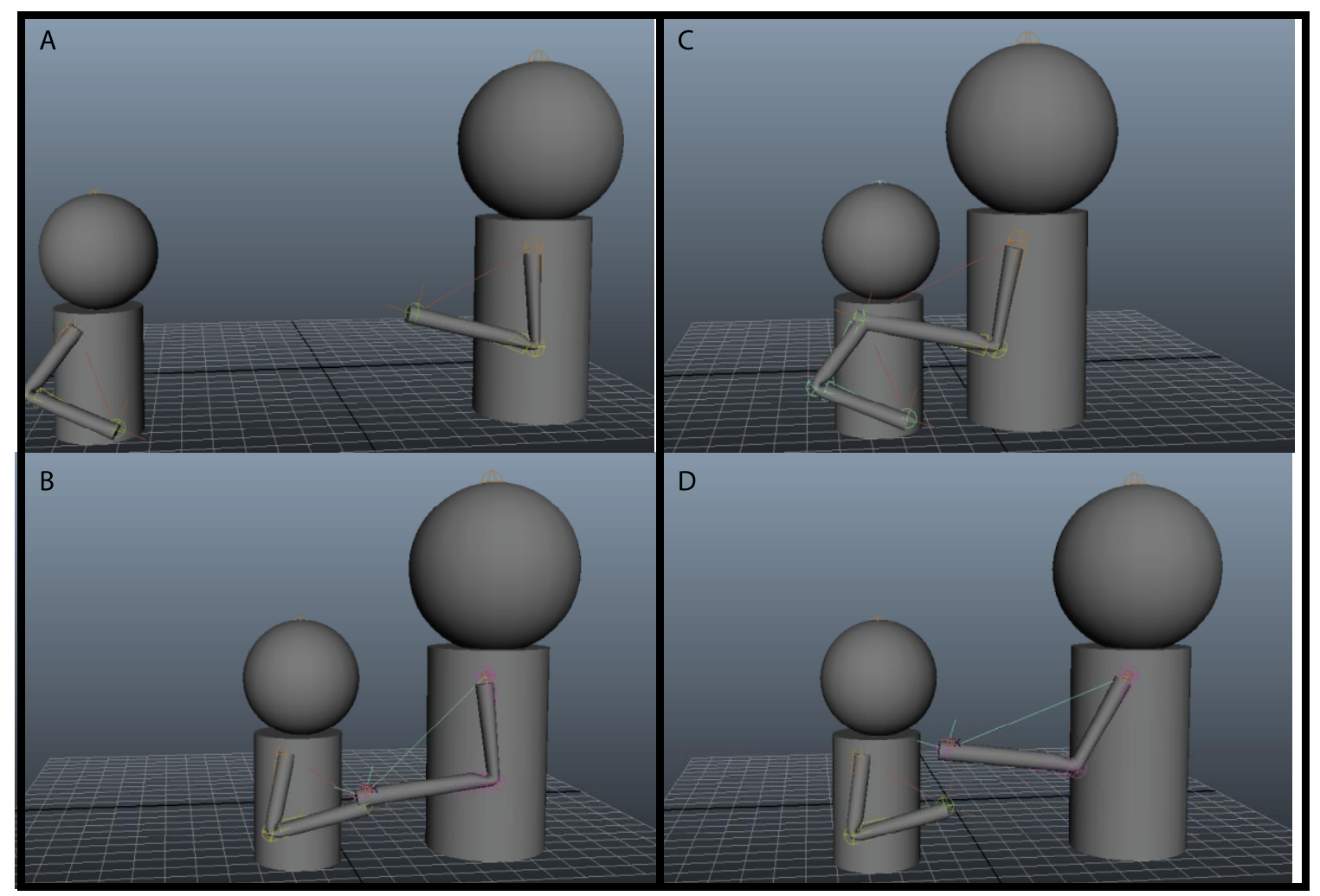

Figure 11. Simulation screen-grabs. Our avatars are simple animated agents capable of 'walking' and moving limbs, and interacting with each other. (A) The agents initialized to start an Episode. (B) The Child pulling the Mother towards him. (C) The Mother completing the Child's goal of Bonding. (D) The Mother responding to the Child's learned gesture.

Our simulations show how a 'beckoning' gesture emerges over time, across a wide range of parameter settings. Figure 11 shows selected frame shots of the interaction of Mother and Child which demonstrate ontogenetic ritualization. Some parameters are artificially selected to yield more realistic patterns of behavior, while others can be systematically varied to achieve faster (or slower) time-courses of gestural acquisition, as well as longer or shorter gestural forms for $\mathrm{C}$. Changes in certain parameters correspond to the speed of M's recognition, and may correlate with sensitivity (or not) to gestural forms and understanding others' goals, while others may correlate with C's ability to quickly learn postures and/or seek out less effortful options for action. Future work could more systematically explore this parameter space, replacing particular parameters with more nuanced control mechanisms, or else seek to relate these parameters, and their possible variation, with available data in primate communication and social cognition. 


\subsection{From LCA-c to early Homo sapiens}

\section{From Pantomime to Protosign and Protolanguage}

We now examine MSH's hypotheses on the transition our distant ancestors made to protolanguage, in the above sense of a communication system that is open to the addition of new "protowords" far more readily than the (almost) closed set of calls or gestures of a group of nonhuman primates - but still lacks the means, beyond mere juxtaposition of two or three protowords, to put protowords together to continually create novel utterances.

MSH hypothesizes that the key transition here was the emergence of a brain and a social niche that could support the use of pantomime (as distinct from the occasional emergence of ape gestures that appear iconic). Pantomime is here the ability to use reduced forms of actions to intentionally convey aspects of other actions, objects, emotions, or feelings. Flapping one's arms could indicate an object (a bird), an action (flying) or both (a flying bird). Examples linked to feelings or emotions could include miming the brushing away of tears to feign sadness. The key point is that this form of pantomime provides an open-ended semantics - any performance can be mimed to represent some associated object, action or situation. Once the brain mechanisms and social understanding for pantomime are in place, it allows a group to consciously communicate information about novel requests and situations that could not otherwise have been indicated.

MSH suggests that brain mechanisms for imitation evolved further to first support pantomime of manual actions and, subsequently, pantomime of actions outside the mime's own behavioral repertoire. The latter would include the "flying bird" example. It would also involve a change of perspective from "something I do" to "something that an other does." Thus an invitation to hunt for deer might involve pantomime of one's actions during the hunt (and thus might engage the whole body, not just the arms and face) or the movements typical of a deer. The former contains no symbol for "deer," the latter no symbol for "hunt" - both rely on context-based inference on the part of the observer (a process still important in language use today).

Not all concepts, situations, commands or invitations are readily pantomimed. A pantomime may be costly to perform if ambiguity is to be reduced and even then may remain highly ambiguous. This creates the selective advantage for brain structures and social practices that support the invention and use of conventional gestures that simplify, disambiguate (e.g., to distinguish "bird" from "flying") or extend pantomime. The result is protosign: a set of 
conventionalized gestures (signed protowords, which we may also call protosigns) used to formalize and disambiguate pantomime. To return to the "flying bird" example, starting from the same pantomime, it requires conventionalization for a community to converge on (differently simplified) separate gestures to represent "flying" and "bird" so that one may later use the new sign for "bird" as part of the expression for "dead bird."

Note that a protosign is likely to emerge from a pantomime through this conventionalization process (similar to the process whereby ontogenetic ritualization reduces a praxic action sequence to a simplified communicative gesture) only if the pantomime is performed often enough and with sufficient specificity to support the conventionalization. Pantomime remains useful in "one off" situations, or where the gesturer does not know the conventional sign (as in the case of children, or an encounter between protohumans from tribes with different protolanguages).

The key point here is that MSH hypothesizes that the path to speech is indirect. There are far more actions, events and objects that can be pantomimed than can be vocally imitated. Monkeys and apes have a limited repertoire of calls and cannot imitate human spoken words, but monkeys and apes have manual dexterity, and apes can learn to imitate some small fraction of manual signs from, e.g., ASL, American Sign Language (but they do not learn ASL since they have no notion of grammar).

Pantomime transcends the slow accretion of manual gestures by apes (as modeled in Section 5) to support a protosign system which - and this is the key point - has an open semantics, extensible to a large set of novel meanings (Stokoe, 2001). MSH hypothesizes that the capability for protosign - rather than elaborations intrinsic to the core vocalization systems - may then have provided the essential scaffolding for protospeech and evolution of the human languageready brain. According to $\mathrm{MSH}$, it is protosign that so dramatically opens up semantics that there is an adaptive pressure to develop a corresponding flexibility in vocal control and learning. We posit that protosign and protospeech are thereafter involved in an expanding spiral, as mechanisms evolved to support one become available to support the other.

In other words, the mechanisms that evolved to support protosign (e.g., in Broca's area plus areas "beyond the mirror") extended collaterals to yield the control of the vocal apparatus that supported an increasingly precise control of vocalization needed to support speech - but this control and the associated learning mechanisms were only adaptive once protosign had built atop 
pantomime to develop the social practice of using conventionalized gestures to support an openended semantics.

\section{The MSH claim that protosign scaffolds protospeech remains controversial}

Aboitiz (2013) and Fogassi, Coudé, and Ferrari (2013) emphasize rudimentary pathways in the monkey that may be homologous to pathways for vocal control in the human. Although such training is hard, monkeys can be trained to vocalize to presentation of food with some neurons in the classic F5 area of the FARS and MNS models becoming activated during the conditioned vocalization (Coudé et al., 2011). However, this remains a difficult task for the monkeys and the articulatory and phonatory components often dissociate (silent vocalization). Fogassi et al. (2013) suggest that perhaps the conditioned vocalization neurons constitute a primitive neural substrate of a cortical center for the voluntary control of vocalization. The issue, though, is this: Do these data promote voice as being the direct route to language? Or do they demonstrate a very restricted path on which future evolution (e.g., post LCA-c) could enable protosign mechanisms to scaffold protospeech?

Aboitiz (2013) - building on an excellent review of macaque circuitry serving gesture, vocalizations, and memory (Aboitiz, 2012) - asks "How did auditory-vocal circuits come to dominate a robust gesture-dedicated circuit, out of nearly nothing?" But this conflates the biological evolution of a brain for which hand and voice are equally flexible for language with the cultural evolution of humans for whom speech became the chosen medium in almost all hearing societies, though gesture remains a major complement to speech. The right question for biological evolution might rather be "How did auditory-vocal circuits come to be on a par with a robust visuo-manual circuit when the latter was far better developed in both LCA-m and LCAc?" In any case, Aboitiz argues that direct cortical control of the subcortical vocalizing system generated an inflection point in human evolution that permitted the explosion of vocal language and human communication. In contrast MSH posits two inflection points: (a) the opening up of semantics in the manual domain (pantomime then protosign) and then (b) the role this played in making vocal expression of the open semantics the adaptive pressure for the emergence of neural mechanisms for vocal learning.

Neither Coudé et al. nor Aboitiz address my key argument, namely that an early form of protosign had to evolve atop pantomime as a manual-based communication system to break through the fixed repertoire of primate vocalizations to yield the open-ended semantics that 
rendered complexification of vocal control adaptive. MSH holds that, once protosign (as distinct from pantomime) gets started, it establishes the cognitive machinery that can then begin to develop protospeech, perhaps initially through the utility of creating novel sounds to match degrees of freedom of manual gestures (rising pitch could represent an upward movement of the hand). This provides the adaptive pressure for increased control over the vocal apparatus. This control could then co-evolve with an ability for vocal imitation in attracting prey and confusing predators and with onomatopoeia to yield other vocal gestures not linked to manual gestures. Over time, an increasing number of symbols would have become vocalized, freeing the hands to engage in both praxis and communication as desired by the "speaker."

Many authors downplay the role of manual gestures in language evolution. (See Arbib, 2012, pp.241-244 for discussion and references.) The "speech only" view, if true, would make it harder to explain the signed languages of the deaf or the use of gestures by blind speakers. But if we reject this view, we must still explain why speech arose and became dominant for most humans a topic which will not be pursued further here. For Aboitiz (2013) and Fogassi et al. (2013), vocalization in non-human primates (presumably descended from LCA-c, but now extinct in modern non-human primates) could have reached partial voluntary control that, in conjunction with gestures, could have had an active role in the emergence of the first voluntary forms of utterances, which they assert to be protospeech. However, I stress that no nonhuman primate has vocal imitation whereas apes do exhibit imitation of manual skills, and so argue that it was protosign-scaffolded control of the vocal articulators that provided the breakthrough necessary to support vocal learning.

\section{The Mirror System Hypothesis 2: From Protolanguage to Language}

As noted above, one of the reviewer's concerns with the previous draft was that omitting an account of the transition from protolanguage to language vitiated the present attempt to characterize the evolution of the "language-ready brain". My original strategy was simply to refer the reader to How the Brain Got Language for my account of this transition, but in response to the reviewer's concern I now offer a summary of Chapters 10 through 13 of the book (adapted in part from the précis given in Arbib, 2013b) - noting, of course, that more detail is provided in the book and in the papers by diverse authors cited therein. This approach to the transition from protolanguage to language looks back to complex imitation but adds a more explicit account of 
generalization across categories of slot fillers and suggests that a construction-based (rather than autonomous syntax of abstract rules) is indeed appropriate (recall Section 1.6).

Section 3.1 argues that in early protolanguages, protowords were "unitary utterances" or "holophrases" signifying a frequent event or other item of importance to the community - thus expressing a meaning that a modern language would convey with a phrase or sentence. The catch was that such a system, lacking a grammar, had no flexibility in combining meanings (other than that provided by context). MSH argues that languages got started through two complementary processes rooted in the processes of complex imitation: fractionation whereby holophrases became replaced by the concatenation of "substrings" that became conventionally associated with "submeanings" of the original, and then the emergence of constructions by allowing a "substring" in the original compound to become a "slot" to be filled by any element from an increasing category which might remain "semantic" or become increasingly "syntactic." In time, protolanguages segued into languages with more or less well-defined construction grammars. The remaining subsections of Section 3 then suggest that the processes which created the earliest languages are still operative today.

Section 3.2 shows how the child acquires language not through direct mastery of an adult grammar but by extracting fragments of the adult speech stream and fractionating them to derive constructions which support more effective communication and which are built upon over the years to better and better approximate the adult grammar.

Section 3.3 documents how similar processes applied in the emergence of two new sign languages, Nicaraguan Sign Language and Al Sayyid Bedouin Sign Language, though here the community is developing new signs and constructions as well as learning and adapting those developed by older members of the community. Nonetheless, we stress that this is very different from the original transition from protolanguage to language because each community contains members who speak Spanish or Arabic, respectively, and thus already have the idea of language (e.g., that words can be combined to express novel meanings) and a stock of ideas (such as "Thursday") which would have been unavailable to our distant ancestors and for which they sought to develop appropriate signs.

Section 3.4 emphasizes that these processes are also responsible for how languages keep changing, whether in the processes whereby two languages fuse aspects of their grammars and lexicons to yield pidgins and creoles, or through the process of grammaticalization whereby 
certain frequently used ways of expressing meaning become ritualized into new constructions that modify the grammar of a given language.

\subsection{How Languages Got Started (Arbib, 2012, Chapter 10)}

An important debate is between differing views of the nature of the earliest protolanguages, and this in turn shapes the debate as to what had to change as languages emerged from protolanguages.

The compositional view (Bickerton, 1995) hypothesizes that Homo erectus communicated by a protolanguage in which a communicative act comprised a few words like nouns and verbs in the current sense, but strung together without syntactic structure. In this view, the "protowords" were so akin to the words of modern languages that languages evolved from protolanguages just by "adding syntax."

The holophrastic view (which I share with the linguist Alison Wray) holds that in much of protolanguage, a complete communicative act involved a "unitary utterance" or "holophrase" whose parts had no independent meaning. A pantomime-scaffolded protosign system escapes from the closed set of innate calls or gestures as holophrases get invented and spread because they signal an event or action or object that is either frequent and noteworthy, or very important even if rare.

How, then, do we get to language on the holophrastic view? We follow Wray in arguing that "protowords" were fractionated or elaborated to yield words for constituents of their original meaning; we go further by arguing that as protowords were fractionated, constructions developed to arrange the words to reconstitute those original meanings and many more besides. Let's consider how this process might work in the domain of pantomime. It is ahistorical, since it concerns doors, and doors did not exist when protolanguage was emerging from pantomime, but let's persevere. If I pantomime "he is opening the door" there will be no natural separation of noun and verb. Suppose that the pantomime for open the door became conventionalized by positioning the hand near the body then making a hand-turning motion followed by a movement of the hand away from the body, while the pantomime for close the door became conventionalized by positioning the hand away from the body then making a hand-turning motion followed by a movement of the hand towards the body. What is crucial to this example is that no part of the pantomimes is a symbol for the noun door or the verbs open or closed. It is only the full performance that conveys the notion of a door being opened or closed. In this case, 
the common element to the two performances is the hand-turning motion and over time this might be fractionated from the two unitary utterances to become the symbol for door, even though it was in no way a pantomime for door when the overall (pre-conventionalization) protoword was originally performed. Secondly, once door is fractionated out, a general capacity for complex action analysis invites one to attend to the rest of the original protoword as a new, complementary protoword - yielding open and close, and this can support complex imitation as a means for supporting this compound communicative action as well as mastering its components.

And now something even more important happens. The similarity between door + open and $d o o r+$ close opens the possibility of generalization to the construction $d o o r+X$ where the slot $X$ can be filled by the protoword for any action one might employ with a door, or the construction $Y+$ open where the slot $Y$ can be filled with the protoword anything that might be opened. (Note how specific slot fillers are at this stage - we are a long way from general categories like noun and verb.) We also see how, even at this primitive stage, protolanguage invites generalizations that might otherwise have never been made -the very existence of $Y+$ open invites one to consider operations on non-doors that are like opening a door and yet (consider opening one's mouth) are nonetheless very different. The power of metaphor seems to be an unavoidable concomitant of the recombination of symbols in novel constructions to express meanings that were not covered in the prior repertoire of holophrases.

But even Alison Wray (1998, p. 48) had concerns about what use a partial grammar consisting of a bunch of constructions might be, arguing that a critical level of complexity is required for a grammar to be useful. I disagree. The trouble comes, I think, from viewing a grammar as providing an all-or-none capacity to express propositions, rather than - initially - as a set of independently useful constructions (constructors) that each have "stand-alone utility." Over time, dependencies may arise between different constructions. Many, but not all, became more or less regularized, with general "rules" emerging both consciously and unconsciously only as generalizations could be imposed on, or discerned in, a population of ad hoc tools. The result: a spiraling coevolution of communication and representation, extending the repertoire of achievable, recognizable, and describable actions, objects, and situations which could be thought and talked about.

As constructions emerge, so do the categories of words which can serve to fill their slots. As generalization integrates varied constructions, these categories may themselves generalize to 
yield relatively abstract categories whose elements can fill the slots of diverse constructions. Thus the categories may range from "highly semantic" (e.g., the set of want-able things) to "highly syntactic" (e.g., the category of nouns in a particular language which includes words-forthings as a particular subset).

Having seen how fractionation of words into meaningful elements, we suggest that a similar process would have yielded, piecemeal, the phonology of a (proto)language - where phonology refers here to the system of combining the meaningless elements of a language as described in the duality of patterning in which meaningless elements (e.g., syllables or phonemes in speech; hand shapes and motions in sign languages) are combined into meaningful elements (morphemes and words). All that is required is the existence of so large a (proto)lexicon that words run the risk of confusion without the invocation of some form of (vocal or manual) phonology. Phonology would at first be piecemeal, as efforts were made to better discriminate the production of similar protowords with distinct meanings. This might lead to a stage in which many protowords were at least, in part, "nonphonological" while meaningless units were exuberantly overgenerated in further conventionalization of other protowords. But this would set the stage for a process wherein the stock of these units and the rules of their combination would be winnowed, while more and more protowords would be reduced to "phonological form."

Saussure (1916) distinguished the signifier from the signified (or words from concepts), but then highlighted the "sign" as combining these with the linkage between them. In assessing $\mathrm{MSH}$, it is crucial to distinguish the mirror system for neurally encoding a signifier (a symbol as an articulatory gesture, whether spoken or signed), from the linkage of the symbol to the neural schema for the signified (the concept, situation, action, or object to which the signifier refers). Parity between speaker and hearer is thus a two-fold process: It rests on the hearer (i) recognizing what word-as-a-phonological-entity the speaker produced and (ii) having sufficient experience related to that of the speaker for the schema assemblage elicited by that recognition to more or less match the speaker's intentions. This interpretation (induced schema assemblage) will be swayed by context and may result from dynamic processes in the hearer's schema network that take him or her well beyond any direct "literal" meaning.

We distinguish a dorsal stream, which includes a mirror system for articulatory expression which, says MSH, evolved from (but is not coextensive with) the mirror system for grasping (via the transition through pantomime to protosign and protospeech) from a ventral network of 
concepts-as-schemas stored in long-term memory (with our current "conceptual content" formed as an assemblage of schema instances in working memory). This dorsal-ventral division is reminiscent of that postulated by Hickok and Poeppel (2004) in their analysis of cortical stages of speech perception, combining a dorsal stream mapping sound onto articulatory-based representations and a ventral stream mapping sound onto meaning (more on this in Section 4)..

\subsection{How the Child Acquires Language (Arbib, 2012, Chapter 11)}

We argue that the same basic mechanisms may have served both protohumans inventing language and modern children acquiring the existing language of their community. These mechanisms comprise (1) the ability to create a novel gesture or vocalization and associate it with a communicative goal; (2) the ability both to perform and perceive such a gesture or vocalization; while (3) commonalities between two structures can yield to "fractionation," the isolation of that commonality as a gesture or vocalization betokening some shared "semantic component" of the event, object, or action denoted by each of the two structures. This could in time lead to the emergence of a construction for "putting the pieces back together," not only allowing recapture of the meanings of the original structures but also with the original pieces becoming instances of an ever wider class of slot fillers. A number of authors (Arbib \& Hill, 1988; Bates \& MacWhinney, 1989; Hill, 1983; MacWhinney, 2005; Tomasello, 2003) have contributed to this view of language acquisition;

This perspective ties in with our original view of complex imitation. It is not the ability to repeat something one has just observed, but rather the ability to add a skill of moderate complexity to one's repertoire after observing another employing it on several occasions, reducing it to an assemblage of variants of actions in the repertoire (or building up new actions by other means). When it comes to modern languages, early mastery of the phonology of a language (something that protolanguages would have lacked before their complexity drove the emergence of duality of patterning) provides a relatively small set of actions that can be used to analyze a novel word on hearing it or seeing it signed, and then use that analysis to perform the word with moderate success in the context in which it was earlier used. Mastery of the nuances of the word's meaning and increased fluency with its pronunciation may then involve further experience, as is the case with any skill. As the stock of words expands, so does it make possible the learning of new constructions, in which a "slot filler" involves not the use of a single word 
but rather the use of a class of words that is defined through use of language rather than being predefined as part of an innate syntax.

But ontogeny does not recapitulate phylogeny. Adult protohuman hunters and gatherers had to communicate about situations outside the range of a modern 2-year-old, and protohumans were not communicating with adults who already used a large lexicon and set of constructions to generate complex sentences. Nonetheless, I argue that biological evolution created the brain mechanisms that made the cultural evolution of language possible in the past and support both language acquisition and the emergence of new languages in the present day.

\subsection{How Languages Emerge (Arbib, 2012, Chapter 12)}

This chapter analyzed the development of two new sign languages: Nicaraguan Sign Language (NSL) which developed in just 25 years within a community of deaf Nicaraguans, and Al-Sayyid Bedouin Sign Language (ABSL). Here we will focus on NSL.

Senghas, Kita, and Özyürek (2004) studied three cohorts (roughly, groups of people who acquired NSL in the first, second or third decade of its existence). In describing a particular bowling ball video clip, many first cohort signers used a single movement for "rolled downhill" akin to a Spanish co-speech gesture in describing a related video, whereas later NSL signers more often expressed manner, roll, and path, downward, in separate signs in succession. Thus, NSL is not a copying of Spanish co-speech gestures (though it should also be noted that many sign languages do express manner and path within a single sign). Yet roll followed by downward might mean "rolling, then descending." Senghas et al. found that NSL signers also developed a way to put the pieces back together again. NSL now has the X-Y-X construction, such as rolldescend-roll, to express simultaneity. The X-Y-X construction appeared in about one-third of the second- and third-cohort expressions they recorded in response to the bowling ball video clip, but it never appeared in the gestures of Spanish speakers. This example shows that the general process, posited to be operative in the evolution of protolanguages, of following fractionation with a construction to put the pieces back together and then be available to combine other pieces as well is still operative in the modern brain when the need to expand a communication system demands it.

The existence of a community provides more opportunities to use signs and choose signs than are available to an isolated individual, so that some signs get lost to the community while others gain power by being widely shared - there is "natural selection by learning." Since knowledge of 
another language is possessed by some members of the community, they seek to translate this knowledge into the new medium (as in the case of signs for days of the week entering the lexicon during the early stages leading up to the emergence of NSL). It is not that Spanish influenced NSL, directly in the form of copied signs and constructions. Rather, the speakers accelerated the development of each sign language because they had the notion that words could be combined to express complex meanings and thus injected their clumsy attempts to express these compound meanings in the realm of gesture. The presence of such performances provided invaluable grist for the mill of language emergence, but the emergence of specific constructions suited to express these compound meanings was internal to the emerging sign language community, just as was the conventionalization of signs to express word-like meanings.

\subsection{How Languages Keep Changing (Arbib, 2012, Chapter 13)}

The two aspects of the chapter relevant here are provided by the discussions of grammaticalization and of pidgins and creoles._Grammaticalization is the process whereby over time information expressed in a string of words or a supplementary sentence becomes transformed into part of the grammar. It provides a crucial engine for language change. This section shows that the approach of Heine and Kuteva (2011) is consistent with the framework MSH has created for the processes whereby languages emerge. But how might processes analogous to grammaticalization operate before languages existed? The key is that such processes do not need a complex grammar to get started. Once fractionation and the compensatory invention of constructions had yielded even a limited set of words and constructions, the effort of expressing in words novel ideas that then enter into the modification of many utterances would have provided fuel for the engine of grammaticalization, an engine that is running today in changing languages around the world.

Having seen some of the ways in which grammar can change over time, we turned to the study of pidgins and creoles to get examples of how a new language can be formed when two existing languages are brought into contact. Different historical circumstances may yield different admixtures of semantic and syntactic features from the two languages. Syntactic features may be inherited from both languages, and there may be no general formula for what will survive into the creole once its speakers no longer have knowledge of a different native language to affect their utterances. 
Both grammaticalization and pidgins \& creoles provide ongoing challenges to their own research communities. They, rather than I, must address such issues as the fact that complexities of language proper are not arbitrary across the diverse languages of the world, as it might be expected to be if each language resulted from cultural evolution in conjunction with social conventions within a particular group. Let me just offer a few comments. First, The World Atlas of Language Structures (Haspelmath, Dryer, Gil, \& Comrie, 2005) shows the immense diversity of different strategies that different languages employ, with particular strategies occurring in languages in very different parts of the world. It seems, then, that some strategies spread by language diffusion, but they can arise independently, I speculate, because there are shared aspects of human cognition that people wish to express in language, and thus certain strategies become likely to emerge not as part of an autonomous syntax but out of a drive to express certain ranges of meaning. It is now well known (Bickel, 2010; Evans \& Levinson, 2009) that although there are essentially no absolute linguistic universals, there are statistical universals - patterns that recur across many languages and across different language families. One approach to this has been offered by Hawkins (1999) who suggested that certain pairs (or more) of constructions may have a high probability of co-occurring in the grammars of different languages as a means to reduce complexity: a parser that works efficiently with one of the constructions will work more efficiently if the language employs the other one(s) as well, rather than some alternative (e.g., the use of postpositions versus prepositions).

I do not claim any particular insight into the details of these disciplines, but it seems to me that the way forward will rest in part on a deeper understanding of the learning mechanisms made available by the language-ready brain as part and parcel of complex imitation as it extends into the domain of communication. Section 3 takes us far enough to see the general plausibility of a set of processes in the language-ready brain which, by supporting fractionation, reconstitution via constructions, and the emergence of categories along a dimension from mainly semantic to mainly syntactic can support not only the transition from protolanguages to languages through cultural evolution, but also of language acquisition by the individual in a community in which the language is well established, and the continuing processes of language change. 


\section{A Double Dorsal-Ventral Perspective on Brain and Language}

Our long-range goal is to update $\mathrm{MSH}$ as the pool of available data begins to fill in details and modify assumptions in the process. However, in this final section, we abandon evolutionary modeling. Instead we focus on the question of "What makes a brain language-ready?" to provide a better-defined target for assessing what it is that evolved. However, Section 4.1 does attend to what has gone before by suggesting how our concern with the visual dorsal and ventral streams in our study of manual actions informs an MSH-based view of the brain mechanisms underlying language: We distinguish a dorsal path supporting the articulation of language from a ventral path supporting the association of meaning with the auditory or visual stream of language. We use a study of how one recognizes speech in a foreign accent to emphasize that MSH does not constitute an endorsement of the motor theory of speech perception.

Section 4.2 emphasizes the role of auditory dorsal and ventral streams in sentence comprehension, but is silent about how language relates to how we interact with the world. The role of frontal cortex is proposed to integrate/evaluate (control) the output of the two auditory streams: the dorsal stream employs syntactic structuring to identify the agent in a sentence (actor computation); while the ventral stream identifies and unifies AE (action-event) schemas to pass a semantic representation to frontal cortex.

Section 4.3 complements Section 4.2 by focusing not on how we decode the speech (or sign language) signal, but rather on "what language is about": how language is related to the description of a visual scene (production) or to the linguistic build-up of a semantic representation on the basis of linguistic input (comprehension). Section 4.3 does this by building upon the scene interpretation system of Section 1.3 to introduce a particular variant of construction grammar, Template Construction Grammar (TCG). The differences between these two models set up some of the research challenges that are the focus of Section 5.

\subsection{An MSH-based view of the Brain Mechanisms Underlying Language}

No matter what the origins of the language-ready brain - e.g., irrespective of whether the evolutionary path to speech is direct or indirect - the fact remains that for most humans their primary language is a spoken rather than a signed language. Thus, in section 4.2 we will summarize a conceptual model of the role of the auditory system in conveying speech signals during the comprehension of spoken language (Bornkessel-Schlesewsky \& Schlesewsky, 2013). 
However, when we speak, much information is conveyed by co-speech gesture of the face and hands - but we can communicate effectively by telephone and so must agree that the auditory channel is the primary channel for the receipt of language. Well, primary and not primary since many people may each day comprehend more words and phrases through reading than through listening. Nonetheless, we understand that reading is a recent invention and that humans did not evolve to be able to read - rather, reading evolved culturally to exploit and reshape brains that had previously acquired a spoken language (Dehaene \& Cohen, 2007; Dehaene et al., 2010; Petersson, Reis, Askelof, Castro-Caldas, \& Ingvar, 2000). Thus the issue of visual input in language comprehension in modern humans is of quadruple interest:

- in understanding sign language (Emmorey, 2002);

- in the role of cospeech gestures in understanding spoken language (Kendon, 2004; McNeill, 2005);

- in reading (Cornelissen, Hansen, Kringelbach, \& Pugh, 2010); and

- in understanding how our use of language may be situated with respect to our perception of the world around us (the visual world paradigm: Altmann \& Kamide, 2009; Knoeferle \& Crocker, 2006; Tanenhaus, Spivey-Knowlton, Eberhard, \& Sedivy, 1995).

We will offer a schema-theoretic approach to modeling the last of these in Section 4.3, but the other three are outside the scope of this article. In the model of 4.2, perception is restricted to the stream of sounds that form the signals for language, not to hearing the sounds of the external world more broadly. By contrast, the model of Section 4.3 is agnostic as to whether the sensory signals are auditory or visual, or whether the motor output is vocal, manual, or multi-modal instead, its focus is on vision in linking utterances to the world (what language is about), whether during perception or production. Section 5 will, among other issues, note the challenge of attempting to synthesize these approaches, more as a stimulus to future research than as an end itself.

To provide background for these models, we next offer some general comments on neurolinguistics, and then present three views of the language-ready brain offered in earlier papers on MSH.

Because spoken language is so persuasive, the auditory system has dominated much of neurolinguistics. Indeed, almost all major neurobiological models of language processing (e.g., Bornkessel-Schlesewsky \& Schlesewsky, 2013; Bornkessel \& Schlesewsky, 2006; A. Friederici, 
2012; Friederici, 2011; A. D. Friederici, 2012; Hagoort, 2013; Hickok, 2012; Poeppel, Emmorey, Hickok, \& Pylkkänen, 2012; Saur et al., 2008; Ueno, Saito, Rogers, \& Lambon Ralph, 2011; Weiller, Musso, Rijntjes, \& Saur, 2009) focus on speech processing and, indeed, usually focus on comprehension rather than production. Our interest in signed language and in production as well as comprehension shows that this is too limited a view - without denying the importance of these speech-centered approaches. Sections 4.3 and 5 represent our larger attempt to integrate MSH with these achievements in neurolinguistics, but much more needs to be done (from both sides).

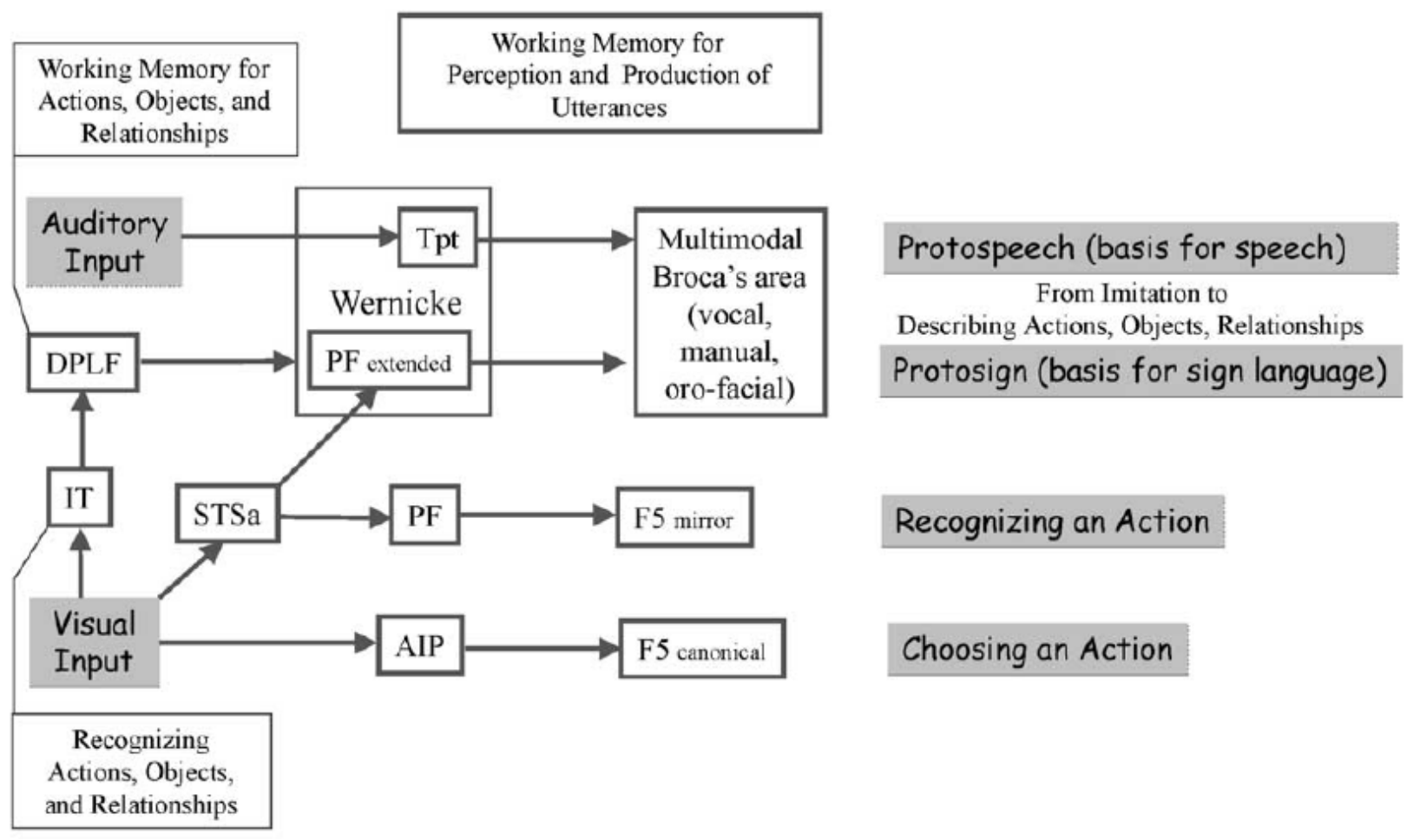

Figure 12: A high-level view of the cumulative emergence of three fronto-parietal systems: choosing an action $\rightarrow$ recognizing an action $\rightarrow$ describing an action in multiple modalities (Arbib \& Bota, 2003, to whom the reader is referred for neuroanatomical nomenclature and discussion). This schematic builds includes multiple working memories in deference to the early hypotheses of Aboitiz and his colleagues (Aboitiz, 1995; Aboitiz \& Garcia, 1997). A major concern of Section 4.3 is to consider the structure in greater detail than considered by Aboitiz et al in these papers or in later work (Aboitiz, 2012; F. Aboitiz, S. Aboitiz, \& R. R. García, 2010b; Aboitiz \& García, 2009; Aboitiz, Garcia, Bosman, \& Brunetti, 2006)

Arbib and Bota (2003) emphasized the importance of seeking homologies between the brains of macaques and humans - to develop a comparative primate neuroanatomy to test and challenge hypotheses about the evolution of the language-ready brain. The NeuroHomology Database 
(NHDB; Bota \& Arbib, 2004) was developed for this purpose but has long been defunct - it is time for NHDB Redux. The second purpose of the paper was to learn from the work of Aboitiz and his colleagues (Aboitiz, 1995; Aboitiz \& Garcia, 1997) - see (Aboitiz, 2012; Aboitiz et al., 2010b; Aboitiz \& García, 2009) for more recent developments in their approach. Perhaps their main contribution was to stress the development in working memory needed to support language. Inspired by Baddeley's account (Baddeley, 2003; Baddeley \& Hitch, 1974), they emphasized the phonological loop (F. Aboitiz, S. Aboitiz, \& R. García, 2010a)- in Section 4.3 we look at our recent, deeper investigation of what Baddeley called the visuospatial sketchpad.

Figure 12 is from Arbib and Bota (2003); the original links the brain regions here to a more anatomical view of cerebral cortex offered by Aboitiz et al.. We see visual input bifurcating into a ventral path (moving up via IT to support actions, objects and relationships) and into a range of dorsal paths, corresponding to choosing an action (recall FARS), recognizing an action (recall MNS) and thence to protosign (conventionalized from pantomime of actions, objects and relationships) and protospeech (which requires new motor control of the vocal apparatus and a related tuning of auditory perception).

"Recognizing an action" occurs twice here. On the dorsal path, the action has to be one in the agent's repertoire, while on the ventral path, it can be any action. The issue can be clarified by considering Buccino et al.'s (2004) fMRI study of human subjects viewing a video, without sound, in which individuals of different species (man, monkey, and dog) performed ingestive (biting) or communicative (talking, lip smacking, barking) acts. In the case of biting there was a clear overlap of the cortical areas that became active in watching man, monkey and dog, including activation in areas considered to be mirror systems. However, although the sight of a man moving his lips as if he were talking induced strong "mirror system" activation in a region that corresponds to Broca's area; the activation was weak when the subjects watched the monkey lip smacking, and disappeared completely when they watched the dog barking. Buccino conclude that actions belonging to the motor repertoire of the observer (e.g., biting and speaking) are mapped on the observer's motor system via mirror neurons, whereas actions that do not belong to this repertoire (e.g., barking) are recognized without such mapping. However, in view of the distributed nature of brain function, I have suggested (Arbib, 2012, p. 142) that the understanding of all actions involves general mechanisms which need not involve the mirror system strongly - but that for actions which are in the observer's repertoire these general 
mechanisms may be complemented by activity in the mirror system which enriches that understanding by access to a network of associations linked to the observer's own performance of such actions. Note, further, that these are representations of what is going on in the world; our further concern is with communicative actions - keeping track of what others, as well as we ourselves, are saying and signing. We see at the top of Figure 12 two boxes for working memory - corresponding to the former (akin to the visuospatial sketchpad - though, indeed, our modeling of the state of the world involves all the senses, not just vision) and the latter (something much richer than the phonological loop, as we shall see below).

Clearly, the above picture is incomplete but it does give some sense of the underlying neuroanatomy of the language-ready brain. Our next partial view comes from Arbib (2006, 2010) - the latter is titled "Mirror System Activity for Action and Language is Embedded in the Integration of Dorsal \& Ventral Pathways."

Our next Figure diagrams the points made at the end of Section 3.1: "We distinguish a dorsal stream, which includes a mirror system for articulatory expression which, says MSH, evolved from (but is not coextensive with) the mirror system for grasping (via the transition through pantomime to protosign and protospeech) from a ventral network of concepts-as-schemas stored in long-term memory (with our current "conceptual content" formed as an assemblage of schema instances in working memory)." When we see or hear a word we can look at it as form, the signifier, devoid of meaning. We can simply write or pronounce it as an action and recognize a similar action made by others, as indeed we do for non-words like gosklarter. However, in normal language use, the articulatory action is linked to a meaning, the signified. Thus a French speaker saying cheval and an English speaker saying horse have in mind the same kind of equine quadruped. My action-oriented view is that the signifieds are realized in the brain as schemas of a human acting in (or abstracted from) its world - and that the linkage between signifiers and signifieds is many-to-one, competitive and contextual, so that appropriate words to express a schema may vary from occasion to occasion, in part because of the context (assemblage) in which the schema instance is currently embedded. The resulting framework is shown in Figure 13 (Arbib, 2006). ‘ 


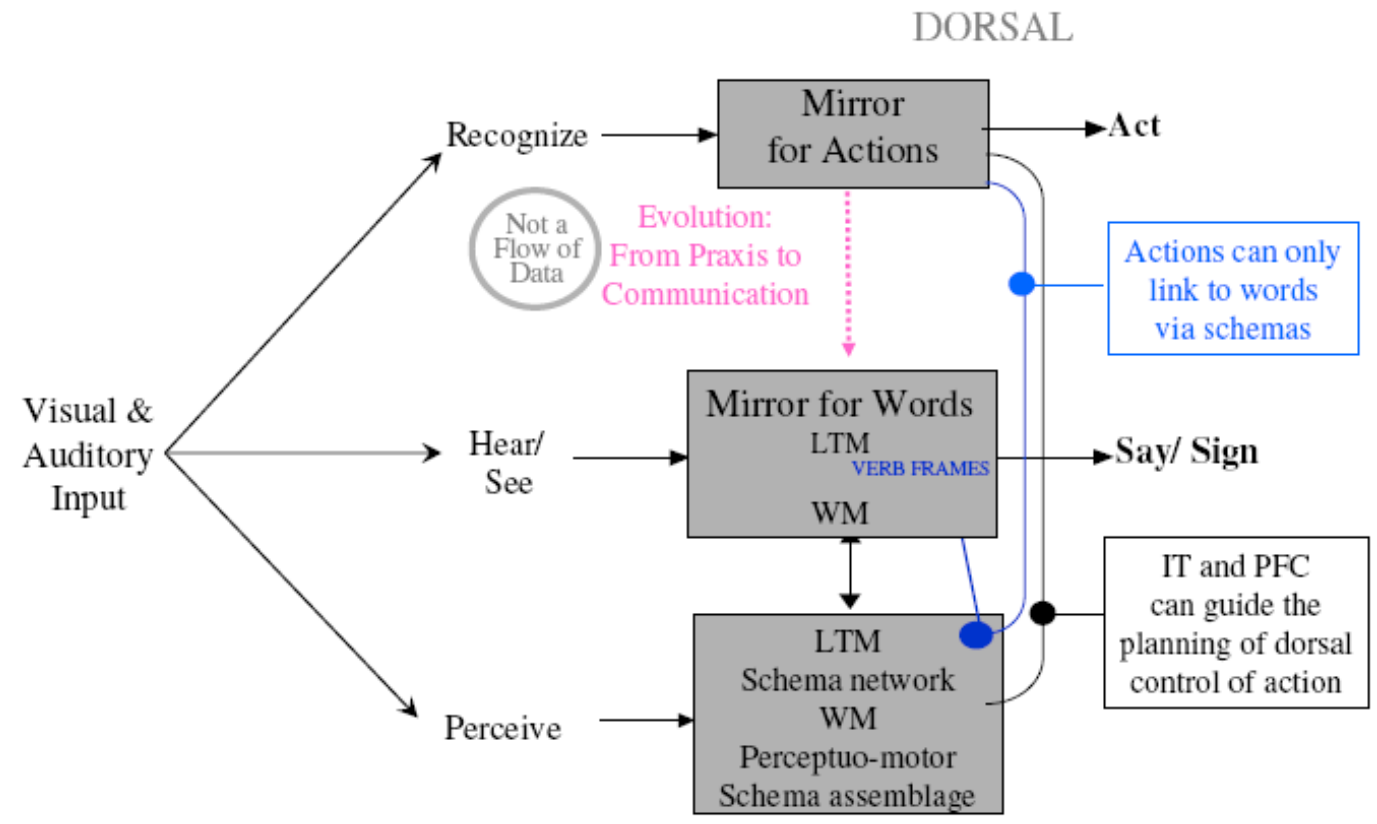

VENTRAL+PFC

Figure 13. In this diagram, each "Mirror" is a dorsal system that integrates mechanisms for performing an action with mechanisms for recognizing the form of the action. Word forms (signifiers) link to schemas related to their signifieds, not directly to the dorsal path for (praxic) actions. The arrow linking the "Mirror for Actions" to the "Mirror for Words" expresses an evolutionary relationship, not (save perhaps for verbs denoting action's in the language user's repertoire) 0 a flow of data. We have two relationships between the dorsal pathway for the Mirror for Actions and the schema networks and assemblages of the ventral pathway and prefrontal cortex. The rightmost path allows inferotemporal cortex and prefrontal cortex to affect the pattern of dorsal control of action (as in the FARS model). The path at left shows that the dorsal representation of actions-in-the-repertoire to conceptual knowledge involves a linkage to verbs via ventral schemas (in this case, motor schemas embedded in a larger schema network).

In Figure 13, each "Mirror" is a dorsal system that integrates mechanisms for performing an action with mechanisms for recognizing the form of the action. Following MSH, we postulate that a "mirror" for phonological expression ("words" as articulatory [vocal or manual] actions) evolved atop the "mirror" for grasping to serve communication integrating hand, face and voice. We also postulate (building on the VISIONS system of Section 1.3) that the concepts - for diverse actions, objects, attributes and abstractions - are now represented by a network of schemas stored in LTM, linking IT and PFC, with our current "conceptual content" formed as an assemblage of schema instances in Working Memory (WM). Analogously, the Mirror for Words 
contains a network of word forms in LTM and keeps track of the current utterance in its own working memory.

The perhaps surprising aspect of this conceptual model is that the arrow linking the "Mirror for Actions" to the "Mirror for Words" expresses an evolutionary relationship, not a flow of data. Rather than a direct linkage of the dorsal representation of an action to the dorsal representation of the phonological form of its signifier, we have two relationships between the dorsal pathway for the Mirror for Actions and the schema networks and assemblages of the ventral pathway and prefrontal cortex (PFC). The rightmost path shown in Figure 13 corresponds to the paths in the FARS model whereby IT and PFC can affect the pattern of dorsal control of action. The leftmost path links signifiers and signifieds. To understand, the leftmost path, note that most words in an utterance are not verbs, and many of these verbs refer to actions outside the speaker/hearer's action repertoire. Thus, even if a few direct connections between signifier and signified can be provided by direct links between the two Mirrors for words that actions-in-the-repertoire, our discussion of Buccino et al. (2004) argues that conceptual understanding (as distinct from motoric correlates) requires linkage to the ventral and prefrontal schema assemblages.

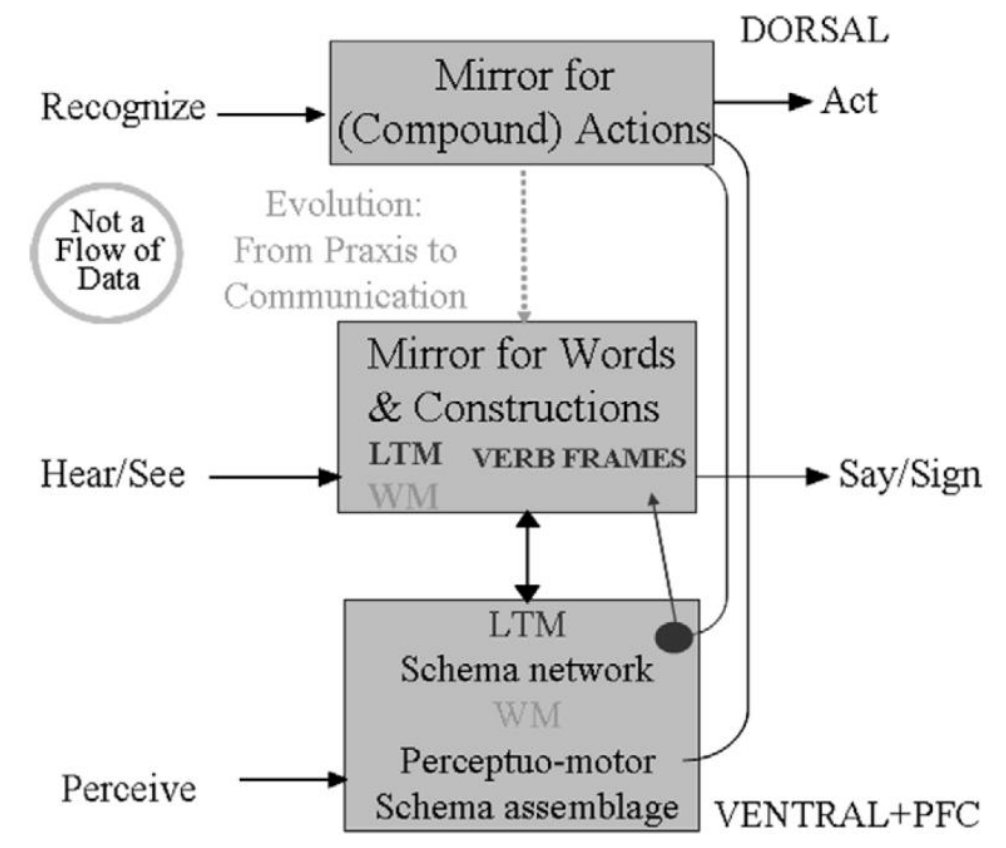

Figure 14. Extending the basic scheme of Figure 13 for single actions and words, we employ complex imitation to lift execution and observation from single familiar actions to novel compounds, and similarly lift words to more complex utterances via the use of constructions. 
Figure 14 (from Arbib, 2010) is based on Fig. 13, but differs from it by adding the word "Compound" to the Mirror for Actions (extending a system for both generating and recognizing single actions) and the words " $\&$ Constructions" to the Mirror for Words (extending a system for both generating and recognizing single words). It postulates that the basic scheme for linking the dorsal supports for single praxic actions to communicative actions [words] via the ventral/prefrontal system can be extended by employing complex action recognition and imitation to lift execution and observation from single familiar actions to novel compounds, and similarly lift words to more complex utterances via the use of constructions. We may see the models of Section 4.2 and 4.3 as two attempts to fill in many details missing in Figure 14; steps toward a synthesis and a brief discussion the challenges such a synthesis poses will then follow in Section 5. However, we close the present section with one more diagrammatic view, in this case focusing on a mirror system for sounds. The aim of the paper from which this is taken (Moulin-Frier \& Arbib, 2013) was to refute the view that (despite an unfortunate suggestion by Rizzolatti \& Arbib, 1998 long since discarded in developing MSH) that MSH rests on the motor theory of speech perception, namely that we only recognize speech sounds through recognition of the detailed motor actions which produce them (Liberman \& Mattingly, 1985). We approached this by developing a model of how we may come to recognize speech in a foreign accent. Figure 15 suggests the implications for this in distinguishing recognizing what has been said (retrieving the articulatory form of the whole word as one would normally pronounce it) from recognizing how it has been said (invoking the articulatory forms on a phoneme by phoneme, rather than word by word basis - as one would do in trying to imitate, rather than simply repeat, the words that one hears). Note that this is seen as a matter of level-of-analysis within the dorsal stream - we still need access to the ventral stream (or so we hypothesize) to extract the full meaning of what we hear. 


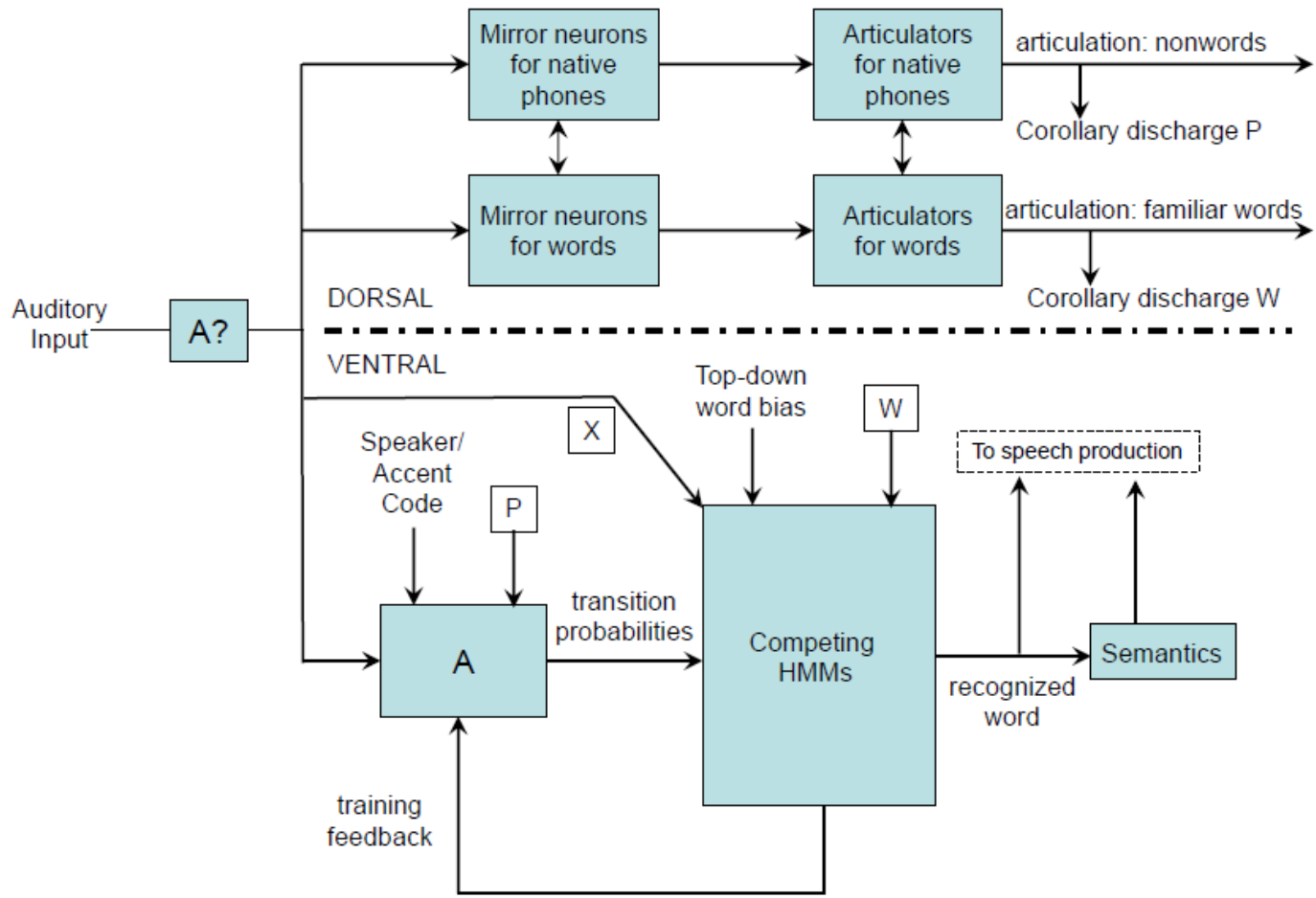

Figure 15. Embedding an accent model (based on Hidden Markov Models [HMMs] in Moulin-Frier \& Arbib, 2013) within a larger dorsal-ventral system for speech comprehension. See text for details.

Box A in Figure 15 - based on the current speaker/accent code for a known accent transforms auditory input to a probability weighted distribution on native phonemes. Section 2 of Moulin-Frier and Arbib (2013) shows that, as training proceeds, the system becomes better and better able to recognize words in a particular foreign accent on which A has been trained, allowing A to transform the auditory input more or less appropriately for that accent. Moving beyond the model of their Section 2, Moulin-Frier and Arbib add pathway X (which may be modulated by discourse context) to record the fact that in some contexts some words may readily be recognized on the basis of part of the auditory input without segmentation down to the level of phone candidates.

Turning to the upper part of Figure 15, we provide a simple view of the dorsal pathway. Here we note that auditory analysis may activate mirror neurons at the level of phones which can then activate articulators for them, and this serves as the basis for imitating non words and novel words. Not shown in the diagram, there are also processes for extending the stock of phones to include approximations to those used in non-native words or native words in a foreign accent. Moreover, in some cases (again, especially with discourse context) auditory input to the dorsal 
path can activate mirror neurons (or circuits) for familiar words and these can in turn drive articulators for these words.

However, the articulation of words may become overt, or may be inhibited while still generating corollary discharge for phones (P) and words (W). We thus make these neural codes available as shown to the dorsal path. If the top contenders among the competing phone candidates are either very close or all very low in estimated probability, then the winner-take-all process among the competitors will be accordingly slow to decide on which word is to be recognized. This leaves time for supplementary processes to take place. The corollary discharges $\mathrm{P} \& \mathrm{~W}$ for the leading contenders can then drive comparison with the auditory feedback which can then tip the balance. This process is especially important when the input is noisy or otherwise degraded.

Finally, we note two other features of Figure 15. One is that both the recognition of a word or the semantics of a word can drive speech production via the dorsal path (recall Figure 13). Secondly (recall the role of cIPS in the FARS model) it may be that the processes described in A are not located within the ventral pathway per se, but instead are located (as suggested by the box A?) in such a way as to provide preprocessed input for both the dorsal and ventral pathways. Such an A? would have the speaker/accent code set to "native" when the task is to imitate, with more or less accuracy, the actual sounds produced by the foreign speaker, whereas it would be set to the code for that speaker/accent if the task is to articulate the word that may have been intended by the foreign speaker, but in the native's accent. Note that the latter task can be accomplished both via the dorsal path alone (when the focus is on decoding the sound of the input word) and via the ventral path when the focus is on the word as a meaningful unit rather than as an articulatory template. The reader is referred to Moulin-Frier and Arbib (2013) for a discussion of how this relates to assessment of the role of mirror neurons in speech perception (Lotto, Hickok, \& Holt, 2009),

\subsection{Auditory Dorsal and Ventral Streams in Sentence Comprehension}

In their analysis of cortical stages of speech perception, Hickok and Poeppel (2004) observe that early stages involve auditory fields in the superior temporal gyrus bilaterally (although asymmetrically) but offer evidence that this cortical processing system then diverges into two streams: A dorsal stream maps sound onto articulatory-based representations while a ventral stream maps sound onto meaning. The latter projects toward the posterior middle temporal gyrus 
which serves as an interface between sound-based representations of speech and widely distributed conceptual representations.

As noted in Section 1.2, related data are available on the macaque auditory system. Rauschecker and his colleagues (Rauschecker, 2005, 2011; Rauschecker \& Scott, 2009; Rauschecker \& Tian, 2000; Romanski et al., 1999), among others, offer evidence for dorsal and ventral streams which process auditory spatial information and auditory pattern/object information, respectively. Both streams eventually project to the frontal cortex, which integrates this information with visual and other modalities. This same anatomical substrate may support both the decoding of vocalizations in nonhuman primates and the decoding of human speech. Complementing this work, a number of authors (A. Friederici, 2012; Hickok, 2012; Saur et al., 2008; Ueno et al., 2011; Weiller et al., 2009) have emphasized the relevance of distinguishing the roles of the auditory dorsal and ventral stream in sentence comprehension. Building on this and their own studies of neural correlates of sentence comprehension in humans adept in diverse languages (e.g., Bornkessel \& Schlesewsky, 2006), Bornkessel-Schlesewsky and Schlesewsky (2013) offer a conceptual model (the focus of this Section) of these roles. Since human speech involves co-speech gestures of face, hand and body, and since many humans can communicate using signed languages with no auditory component, integration of such an approach with models addressing the visual streams and the quest for a deeper understanding of the relations between the two pairs of streams pose important research challenges.

Bornkessel-Schlesewsky and Schlesewsky (2013), henceforth B\&S, represent the structure of their model as in Figure 16(a) but we shall see that Figure 16(c) more truly captures the model as they describe it. Along the way, we will identify both strengths and shortcomings of the model. B\&S dissociate syntactic structure-building and sentence interpretation as follows:

- the ventral stream performs unifications of conceptual schemas (for B\&S these are ActionEvent schemas, AE-schemas) to create objects of increasing complexity.

- the dorsal stream engages in the combination of elements, subserving both syntactic structuring and a linkage to (articulatory) action.

- frontal regions accomplish general aspects of cognitive control in the service of action planning and execution rather than linguistic processing.

Actually, frontal regions in their model accomplish cognitive control by influencing the dorsal and ventral contributions to comprehension. They briefly discuss the role of pragmatics in 
planning and execution of the response to a sentence but this topic lies outside the scope of their model.

They dissociate syntactic structure-building, which they refer to as time-dependent, from sentence interpretation, which they view as time-independent, but I will not follow their notion of time-independence since sentence comprehension involves the building of a semantic interpretation word-by-word and thus is as time-dependent as the building of syntactic structure - the temporal orderings of the words in The painter kissed the girl and The girl kissed the painter change our interpretation of who is the active agent of the kissing. The alternative terminology of "permutation dependent" versus "permutation independent" processes is equally ill-matched to address the distinct interpretations of the above sentences.

Moreover, although Figure 16(a) shows frontal cortex as driven in a feedforward fashion by both streams, the full description of the B\&S model does involve top-down control from frontal cortex. I thus find their distinction of "general aspects of cognitive control in the service of action planning and execution rather than linguistic processing" less than useful, since different languages must require different language-specific processes of cognitive control for these topdown processes to be effective.

Inspired by Rauschecker (1998), they stress the importance of hierarchical processing in the sense of sensitivity for increasingly complex sets of feature combinations within neurons or neuronal assemblies. This sense (which applies, e.g., to the classic Hubel and Wiesel (1977) model of early visual processing) is not to be confused with the sense of a hierarchy of constituents in syntax. I think B\&S have made the wrong choice here. Yes, in processing a single word, the layer-by-layer processing starting from frequency processing in the auditory periphery (or starting from the visual periphery for sign language) and yielding neural codes categorizing basic sound patterns (e.g., monkey calls or human words) is crucial. But this processing plays no role in the B\&S analysis - rather, their model concerns how syntactic and semantic structures are built up once word forms and morphemes are available. Moreover, since they agree that the dorsal stream is involved in mimicry (shadowing of speech in the input accent; recall our discussion of Figure 15), it is strange that they allocate phonemic processing to the ventral stream alone. Indeed, since mimicry is separate from syntactic processing, their postulate that there is a unique function for each stream seems mistaken. Preliminary analysis of the text of B\&S model suggests that it is better represented by Figure 16(b). 


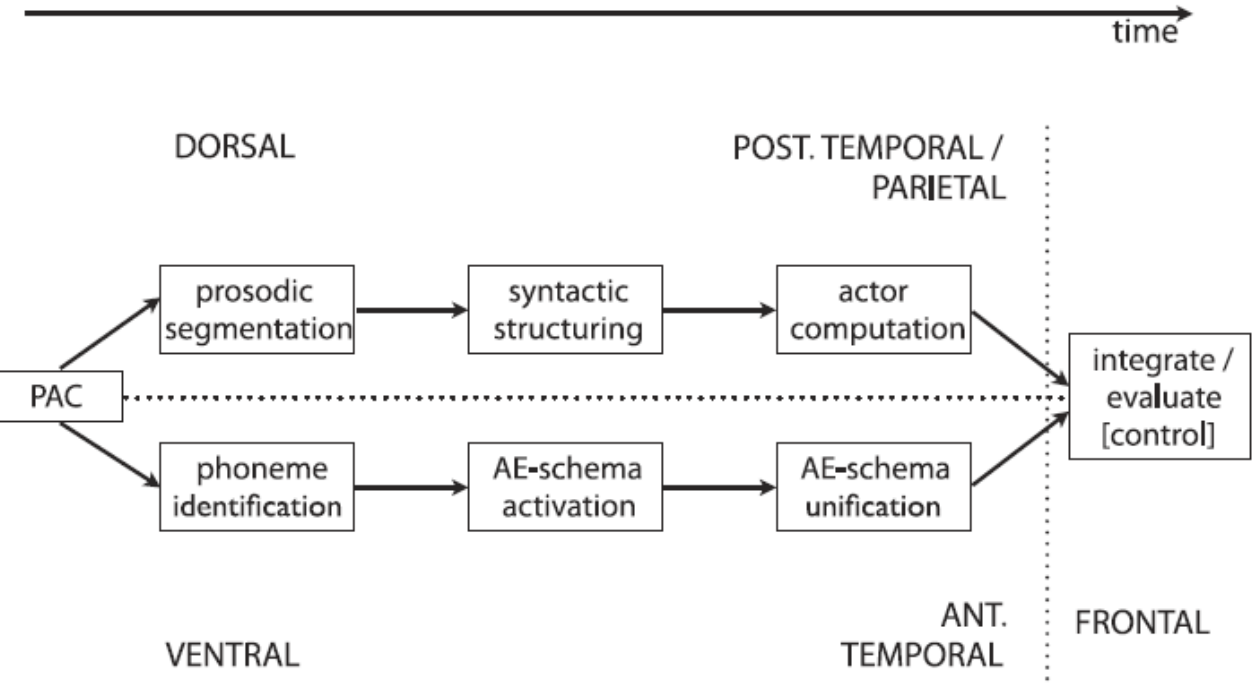

(a)

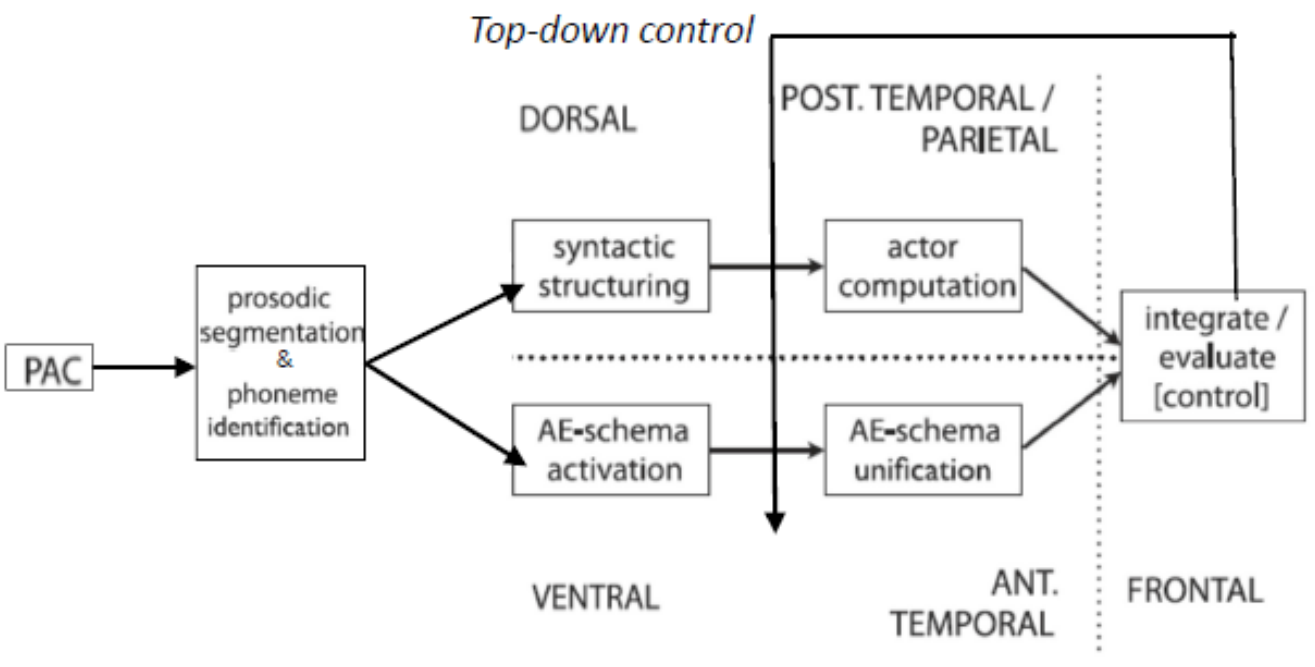

(b)

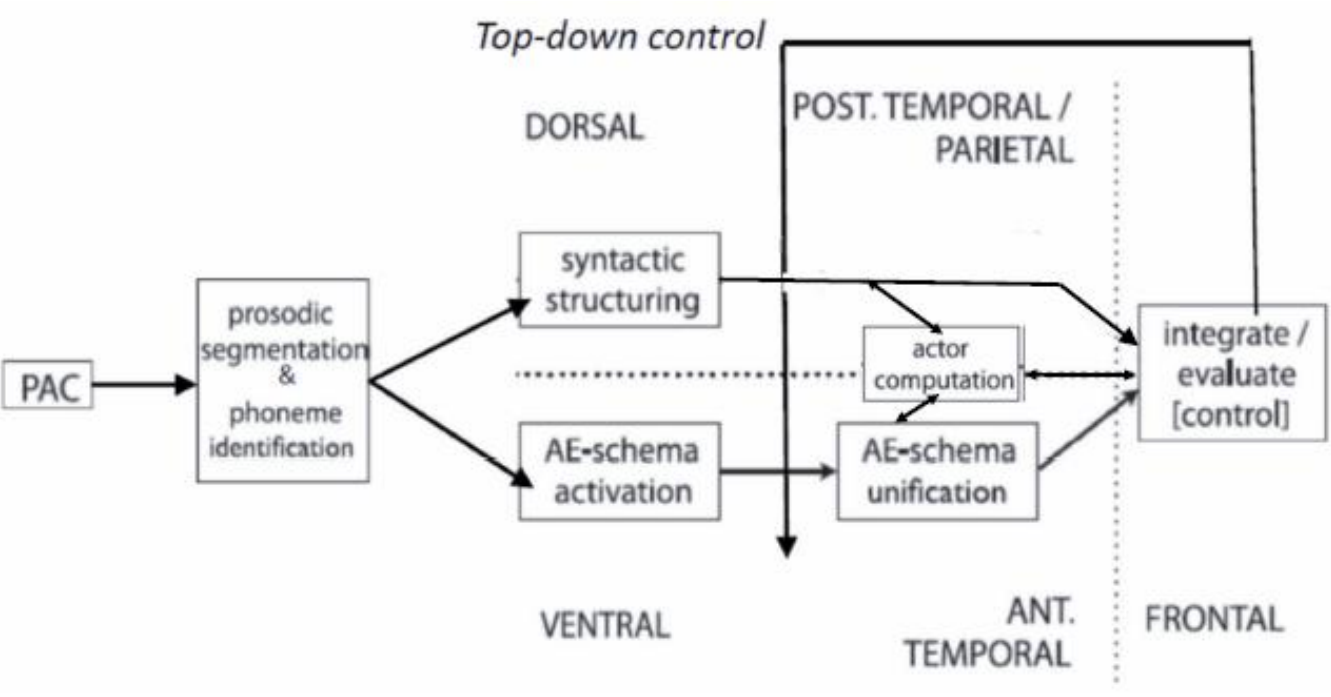

(c) 
Figure 16. (a) In the B\&S model, both ventral and dorsal streams are assumed to emanate from primary auditory cortex (PAC), with their processing converging in frontal cortex (Bornkessel-Schlesewsky \& Schlesewsky, 2013, Figure 1B). The dorsal stream is responsible for syntactic structuring, while the ventral stream finds and unifies Action-Event (AE) schemas. The time axis may be relevant to the processing of a single word, but is misleading in the larger context of the interpretation of a sequence of words as a sentence. (b) A modified view of the B\&S model making more explicit the processing described by Bornkessel-Schlesewsky and Schlesewsky (2013). The auditory preprocessing that yields word and morpheme forms is not really part of the B\&S model and so is treated here as an unanalyzed precursor of dorsal processing of syntax and ventral processing of semantics. The revised figure also makes clear that frontal cortex is posited to exert a top-down influence on these processes. Sentence production and other roles for the dorsal and ventral streams (e.g., a dorsal role in mimicry) are not included in the model. (c) A view making clear that frontal mechanisms may need access to all syntactic structuring and not just actor computation, while suggesting that the latter involves both syntactic and semantic processing.

\section{The role of frontal cortex: Integrate/evaluate [control]}

For $\mathrm{B} \& \mathrm{~S}$, the computations for the ventral and dorsal streams are carried out in the temporal lobe. The two streams converge in frontal cortex which for them is primarily the left inferior frontal gyrus (LIFG); the role of prefrontal cortex (PFC) is not considered. Frontal cortex performs cognitive control and conflict resolution (Novick, Trueswell, \& Thompson-Schill, 2005) - more specifically, reconciling the syntactic representations generated by the dorsal stream with the semantic representation generated by the ventral stream. ${ }^{1}$ Contra $\mathrm{B} \& \mathrm{~S}, \mathrm{I}$ see reconciling syntactic and semantic representations (to be detailed in the next 2 subsections) as very much part of "linguistic processing proper" even if it involves a specialization of more general mechanisms of cognitive control. A key challenge, of course, is to be precise about what we talk about when we talk about semantics and semantic operations. Semantics could be construed as a purely linguistic set of operations (e.g., handling logical form) or could link to "meaning in the world" as is attempted in Section 4.3. We shall shortly see the B\&S approach in the form of AE-schemas.

$\mathrm{B} \& \mathrm{~S}$ assume that cognitive control mechanisms in frontal cortex are structured in a hierarchical anterior-to-posterior gradient from fronto-polar cortex to premotor cortex. Here they

${ }^{1} \mathrm{~B} \& \mathrm{~S}$ see this resolution as a prerequisite for action planning and execution via the premotor cortex viewed as playing a dual role in (a) in the dorsal pathway's auditory-to-motor mapping, and (b) as part of frontal control structures. They do not discuss parietal regions save to suggest "that they play an important role in linking individual sentences to the broader discourse via further relational categories at the sentence level and an interaction with attentional" 
seem influenced by the claim (Koechlin \& Jubault, 2006) that the posterior portion of the prefrontal cortex, including Broca's area and its homolog in the right hemisphere, contains a system of executive processes that control start and end states and the nesting of functional segments that combine in hierarchically organized action plans. For B\&S, "less local (more anterior) control signals can override more local (more posterior) ones in determining action planning and execution. Thus, pragmatic requirements can override literal meaning." However, the possible use of pragmatics in determining how to respond to a sentence is outside the scope of the B\&S model per se (and outside the scope of the TCG model of Section 4.3). Thus our task here is to chart the role of frontal cortex in $\mathrm{B} \& \mathrm{~S}$ for the core challenge of charting how semantic and syntactic processes are combined to extract the "literal" meaning of a sentence.

$\mathrm{B} \& \mathrm{~S}$ argue that frontal cortex "allows for top-down feedback based on the convergence of both streams, which can modulate the processing of the next input item (word)." I agree, which is why it is unhelpful to call the streams independent and diagram only the feedforward (downstream) paths as in Figure 16(a). For example, "the prediction for an upcoming word category that stems from syntactic structuring within the dorsal stream can be used to constrain AE-schema unification for the next word within the ventral stream, specifically whether an element is assumed to play a predicating ("what") or a referential ("who" or "with whom") role." This quote raises two questions. (i) How does the "who" noted here relate to the "what" traditionally associated with the ventral pathway? The answer may well draw on earlier discussions of argument structure by Bornkessel-Schlesewsky and Schlesewsky which they do not draw upon in the B\&S model. (ii) What happens to their neat separation of syntax and semantics if we turn to construction grammar, where each construction combines form and function? Section 4.3 offers a partial answer in its separation of heavy semantics (world knowledge) from light semantics (implicit in the categories of slot fillers for diverse constructions) - suggesting a different dichotomy from that employed by B\&S. Much work needs to be done to resolve this difference,

Continuing with this point, note that it is only at the end of their paper that BornkesselSchlesewsky and Schlesewsky (2013) state that "A prediction of our account is that successful language comprehension requires a binding of the [syntactic] representations computed within the postero-dorsal temporal pathway with the [semantic] representations computed within the antero-ventral temporal pathway." The notion of such binding was not considered earlier in the 
article. It is not a prediction but a new assumption It is an important assumption, but is a promissory note for future research, not part of the B\&S model. One may note the model of Baggio and Hagoort (2011) for suggestions in this direction. Their Figure 3 traces a processing cycle subserving word meaning comprehension in the left hemisphere language network. Inputs are conveyed from sensory regions (visual cortex in their case since words are being read, not heard) to the inferior, middle and superior temporal gyri, where lexical information is activated. Signals are hence relayed to the inferior frontal gyrus, where neurons respond with a sustained firing pattern. Signals are then fed back into the same areas in temporal cortex from where they were received. This loop defines a recurrent network which allows a context to be developed during subsequent processing cycles, with incoming words to be unified within the context.

The dorsal stream: Syntactic structuring $\rightarrow$ actor computation $\rightarrow$ frontal cortex

Although Bornkessel-Schlesewsky and Schlesewsky (2013) assert that the dorsal stream processes sound sequences within both production and comprehension, the B\&S model develops only the comprehension component. The dorsal stream is engaged in (i) the segmentation of the input into prosodic words, (ii) the combination of these elements into a syntactic structure, and (iii) the assessment of the elements in this structure in action-related terms ("who is responsible for the event described"). Since the ventral stream also requires segmentation of the input into words, Figure1(b) treats it as a function whose apportionment between the ventral and dorsal streams is not essential to the B\&S model. Another problem with Figure 1(a) is that syntactic structuring is shown as communicating only the result of actor computation to frontal cortex. Yet B\&S state that "the prediction for an upcoming word category that stems from syntactic structuring within the dorsal stream can be used to constrain AE-schema unification for the next word within the ventral stream."

Since "determining the sequential order of categories within successively larger syntactic constituents ... is a crucial prerequisite to actor identification even though it is not the only determining factor for this operation," B\&S assert that "the position of the actor-identification / action-understanding step as the final mechanism of understanding within the temporal part of the dorsal stream follows from the fact that a sentence participant's position within a sentence is one of several cues to actorhood." Elsewhere, Bornkessel-Schlesewsky and Schlesewsky (2009; Bornkessel \& Schlesewsky, 2006) have presented their extended argument dependency model (eADM) which postulates that core constituent processing proceeds in three hierarchically 
organized phases: (1) constituent structure building without relational interpretation, (2) argument role assignment via a restricted set of cross-linguistically motivated information types (e.g., case, animacy), and (3) completion of argument interpretation using information from further domains (e.g., discourse context, plausibility). This basic architecture is assumed to be universal, with cross-linguistic variation deriving primarily from the information types applied in phase 2. Perhaps (1) and (2) map to the dorsal and ventral streams in B\&S, while (3) corresponds to frontal processing. Figuring out the best synthesis of the two models is a topic for further research, but the point here is that a key use of animacy in eADM is to determine the actor or agent of an event. This may be at the root of the emphasis on actor computation in the B\&S model, but in fact the syntactic role of almost every word is relevant to the part it plays in developing semantic structure. Moreover, it seems that phase 2 is based on the commonly held view that argument role assignment is as much a semantic as a syntactic function, so such role assignment in general (and actor computation in particular) might better be viewed as a computation that can involve activity in both streams. In any case, Figure 16(c) preserves the privileged role of actor computation in the B\&S model while suggesting that this involves both syntactic and semantic processing; moreover the new figure gives frontal mechanisms access to all syntactic structuring (thus being able to make it all available to the ventral stream).

With this, we can turn to how B\&S characterize syntactic structure building. Given the posited role of frontal cortex in coupling the streams, we cannot accept their "cross-linguistically motivated view of syntactic structure as logically independent of sentence-level interpretation" (Bornkessel-Schlesewsky \& Schlesewsky, 2009). In any case, B\&S posits that syntactic structure-building involves the establishment of linguistic categories structured as simple, binary-branching branching trees that do not involve syntactic movement. Here I would only note that there is nothing in the exposition of the B\&S model which demonstrates that the binary trees employed here are superior to the not necessarily binary syntactic frames employed by Hagoort (2005) as an informal application of the explicitly computational model of Vosse and Kempen (2000) of the unification of these frames (one per word in the input sequence) to form syntactic trees. Hagoort and B\&S localize the unification process in different regions - for Hagoort the left IFG is the core unification area for both morphosyntax/syntax/and semantics while for B\&S it plays only a control role - but adjudication of this disagreement is outside the scope of the present review. 
The ventral stream: AE (Action-Event) schema identification $\rightarrow$ AE-schema unification $\rightarrow$ frontal cortex

The truly distinctive process concept in the B\&S model is the notion of Action-Event (AE) schemas and their unification to provide a semantic interpretation of a sentence. These AEschemas (Figure 17) provide word-level semantic information as well as idiosyncratic lexical restrictions; and are posited to allow for schema combination via unification within the ventral stream. Frontal cortex enables syntax needed to help resolve ambiguities in the assemblage of AE-schemas.

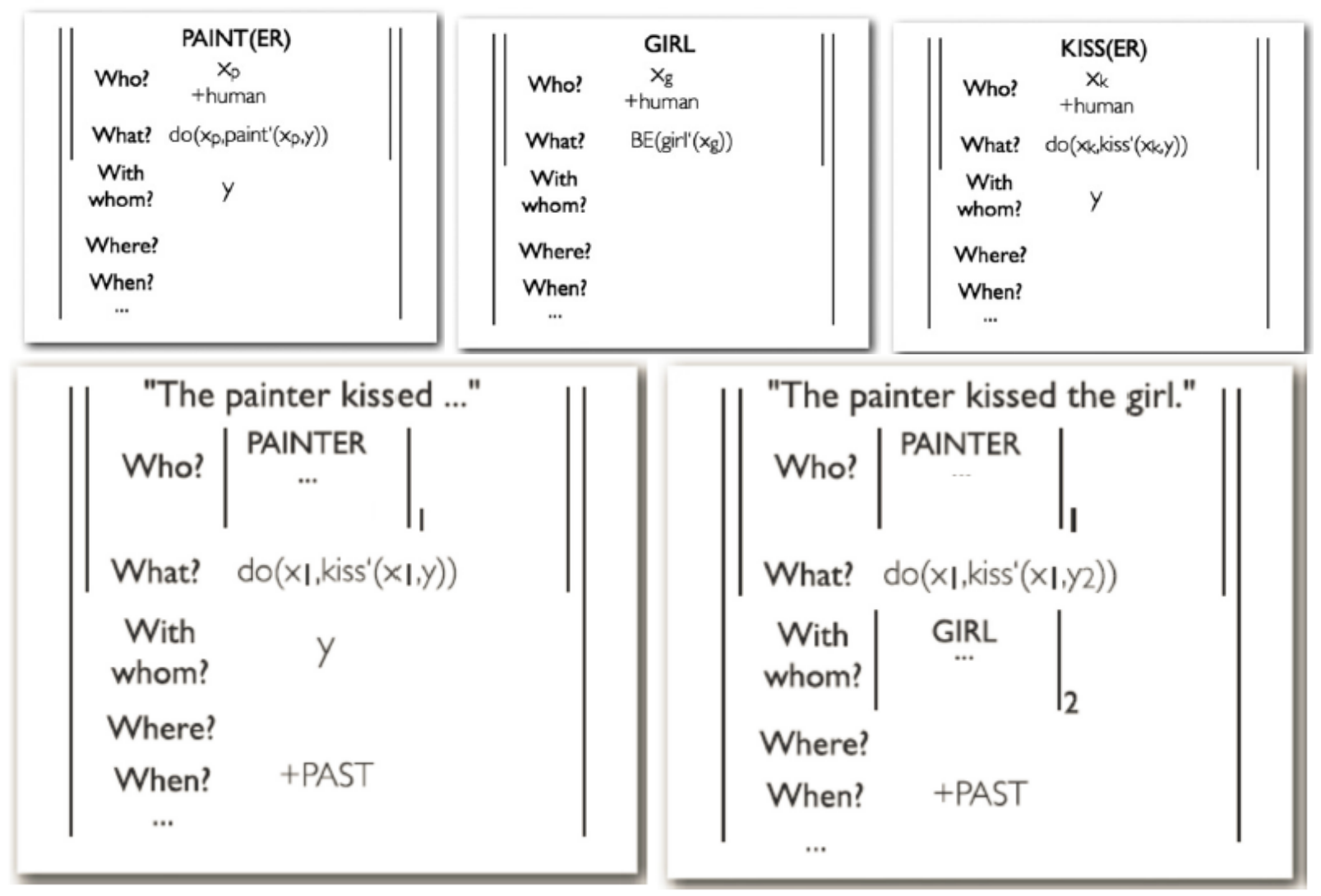

Figure 17. (Top) Three AE-schemas. Note that the same schema serves for both the action paint and the agent painter; similarly for kiss(er). (Bottom) Two steps in unification while processing the sentence The painter kissed the girl. See the text for details. (Adapted from Figures 2 and 3 of Bornkessel-Schlesewsky \& Schlesewsky, 2013.)

$\mathrm{B} \& \mathrm{~S}$ assume that AE-schemas are category neutral and actor-centered:

Category neutrality means, for example, that the "paint" schema in Figure 17 (top) applies equally to the verb to paint and the noun painter. In the verb case, the "what" part of the schema, 
which describes the action or state of affairs, is relevant; in the noun case, by contrast, it is the "who" part of the schema, i.e. the person or thing performing the action or implicated in the state of affairs. The motivation for assuming category neutral schemas of this type is threefold but let's just focus on one, namely that not all languages show a lexical distinction between different word classes, i.e. some languages allow for the same lexemes to be used as "verbs" and as "nouns" as is the case for category-ambiguous words (e.g. cut, train) in English. But surely the real point, given the strict B\&S distinction between syntax and semantics, is that an AE-schema defines a meaning irrespective of the word used to express it. For example, one should not confuse the concept labeled by the English word "painter" with the word itself - presumably it could just as well be labeled by the translation of "painter" into any language or even by a picture of a painter - in which the painter, the act of painting and the resultant painted object are inseparable. The binding to, e.g., English syntax is what separates the agent from the action from the patient in the picture. However, B\&S chooses to combine the agent and the action in one schema and - as we shall see - this poses problems.

Actor centrality reflects the notion that the language comprehension system attempts to identify the person/thing primarily responsible for the state of affairs being described (the "actor") as quickly and unambiguously as possible, resulting in a preference for both actorinitiality and actor prototypicality, i.e. the first sentence participant encountered is interpreted as the actor if at all possible. ${ }^{2}$ Bornkessel-Schlesewsky and Schlesewsky (2009) explain this overall pattern of results by assuming that sentence participants compete for the actor role. Accordingly, in $\mathrm{B} \& \mathrm{~S}$ AE-schema unification involves an actor preference such that a participant will be preferentially integrated into the "who" (i.e. actor) slot if this slot is not already filled. However, a model of sentence interpretation must also be equipped to handle the many sentences which do not have an agentive "who" as subject. It is for this reason that I structured Figure 16(c) to accommodate Bornkessel-Schlesewsky and Schlesewsky's (2012) closing observation (not developed in the body of the paper) that "the preference to fill the actor (who) slot in accordance with the actor-oriented nature of the schemata... could very plausibly result in deficits in the comprehension of non-actor-initial sentences - irrespective of any purported levels of syntactic complexity."

2 This comment is fine for SOV and SVO languages (D. Kemmerer, 2012) but one must note (Dryer, 2011) that $11 \%$ of languages with a preferred order of S, O and V are not S-initial. 
The top row of Figure 17 shows three AE-schemas. In each case, there are slots for Who?, What?, With whom? and more. The schemas for PAINT(ER) and KISS(ER) can be evoked either for the semantics of a noun for one who conducts an action (e.g., PAINTER) or a verb for the action (e.g., PAINT) - we are not given the semantics for the nouns for PAINT and KISS as "objects." The AE-schema GIRL is a "noun only" semantics, with a BE formula in the What? slot.

The bottom row of Figure 17 gives us a sense of how AE-schemas are unified. Here the sentence is "The painter kissed the girl," though the role of the determiner is not included in this analysis. "The painter" evokes the PAINT(ER) schema, but with the presumption that it represents the Agent of the sentence. When "kissed" is read, this presumption is exhibited by placing the AE-schema - now flipped to the label PAINTER - into the Who? slot of the KISS reading of the KISS(ER)schema, with the When? slot filled with +PAST. Finally, when "the girl" is read, the GIRL schema is inserted into the With Whom? slot of the growing schema assemblage. Recall here my earlier rejection of the view that process is time-independent - the temporal ordering of the words in The girl kissed the painter would assemble the same schemas but yield a different assemblage. And how would the above schema handle the sentence The girl painted the painter?

$B \& S$ state that this unification reflects (a) the preference for the initial participant to be interpreted as an actor, and (b) the actor-action compatibility preference, i.e. the tendency to expect a particular actor to perform a semantically compatible action (e.g. for a painter to paint). But (b) is not what happens! Here the painter does not paint, s/he kisses! In any case, there is a problem of distinguishing the "characteristic activity" - to paint - from the current activity - to kiss. In some sense the former is world knowledge. And, of course, we have cases where the actor and the action word form diverge - e.g., author as distinct from writer, so hybrid forms like PAINT(ER) seem the exception rather than the rule. Moreover, B\&S say that unifying the KISS schema with the PAINT(ER) schema requires that the action representation $\operatorname{do}^{\prime}(\mathrm{x}($ paint' $(\mathrm{x}, \mathrm{y}))$ be replaced with do'(x(kiss'(x,y)). But this negates the whole point of including do'(x(paint'(x,y)) "within" the AE-schema rather than relegating it to the cloud of world knowledge.

For B\&S, no semantic integration takes place between the different schemas when schema unification fails. Instead, the model posits that the ventral stream passes unintegrated schema representations on to frontal cortex, where (i) conflict resolution is attempted; and (ii) 
interpretation proceeds following general communicative requirements as applied to the associated schema representations. The issue, then, is how do generic control mechanisms become specialized as we learn skills, with manual praxic skill being different from signed language and even more so from spoken language/ But such questions take us far beyond the B\&S model.

To close this exposition of the B\&S model, I stress that the full paper contains a wealth of suggestions on how specific processes might map to specific brain regions in a way that addresses a range of neurolinguistic data, but my concern here has been to lay bare the key processes of the model to expose certain lacunae that must be addressed in future development of the model. The fact that I do not present so detailed a critique of my own group's model in the next section is in no way a claim of superiority. Rather, I stress the complementary aims of the two models as a platform for making explicit the challenge of developing a neurolinguistic processing model of both production and perception of language in the context of the visual world paradigm.

\subsection{Visual Dorsal and Ventral Streams in Defining "What Language is About"}

We now turn from two streams for audition (how does the speech stream engage with processes of sentence comprehension) to two streams for vision - but vision here is not the vision of sign language or of cospeech gestures but the vision of what a sentence may be about, as demonstrated in the visual world paradigm (though Figures 13 and 14 offer a high level view of how MSH posits integration of these two approaches). In this section, we introduce SemRep as a "semantic representation" that captures the basic elements and relationships in a scene or episode and Template Construction Grammar (TCG) as an approach to both the production of descriptions of visual scenes, and as a "semantic target" for sentence comprehension.

Understanding the language system requires understanding not only the processing of grammatical constraints but also how such processes are integrated with evolutionarily more conserved systems that support our sensory-motor interactions with the world. In this section, we focus on the relation between vision and language. The VISIONS scene understanding system of Section 1.3 provides an example of how a schema network can be used to model some portion of world knowledge (how to recognize objects from low level perceptual features extracted from an image, and how they are relate spatially to their parts or frequently associated objects) and how this knowledge can be retrieved on the basis of activation values of already instantiated schemas. 
In the so-called visual world paradigm, psycholinguistic studies record the eye movements of a subject who is processing linguistic stimuli while simultaneously looking at a related visual scene. Altmann and Kamide (1999), among others, showed how subjects could incrementally combine linguistic, visual, and world knowledge information during a comprehension task. Faced with visual display showing a cake, two toys, and a boy, for example, the subjects were faster to fixate the cake upon hearing the verb "eat" in the sentence "the boy will eat ..." than upon hearing the more general verb "move" in the sentence "the boy will move ....". The challenge is therefore to link language processes to the perception of "realistic" visual scenes while also accounting for the role of world knowledge.

In a visual scene description task, a subject simultaneously gathers information from the image, fixating elements that become salient based on both bottom-up features and top-down hypotheses, and starts generating linguistic output based on relevant visual information. Under pressure to communicate she might start producing fragmented utterances while in more relaxed conditions the message might become composed of well-formed sentences packaging content collected through many fixations. Turning to comprehension, a patient suffering from brain lesions impairing her capacity to use grammatical cues can compensate in some cases by relying on her knowledge of the world to assign linguistic inputs to their correct semantic role and correctly identify what picture matches the sentence she has just heard.

It is not claimed that scene description is the be-all and end-all of language; it is claimed that it provides a basic case for understanding how world and language are linked. The same methodology could and should be extended to look at the interpretation of questions and commands within a given context. A much greater challenge (which we have not yet addressed) is to extend the methodology beyond the here and now.

Arbib and Lee (2008) introduced SemRep as a hierarchical graph-like "semantic representation" of a visual scene, whether static or dynamically extended over time (an episode). A single scene can have many SemReps. Each SemRep encompasses one analysis which captures a subset of the agents, objects, actions and relationships that may be present in the one (temporally extended) visual scene. SemRep may be viewed as an abstraction from the assemblages of schema instances in the WM of VISIONS (Section 1.3), but with the crucial addition of actions. Note that dynamic scene analysis takes us beyond the analysis of single images. If we see a picture of a man with a woman's hand flat against his cheek, and we have no 
cues from his facial expression, we cannot tell whether the scene is "woman slapping man" or "woman stroking man's cheek" unless we can see the temporal sequence of which this is part. The MNS model of action recognition in Section 2.1 (Bonaiuto et al., 2007; Oztop \& Arbib, 2002) is based on recognizing the temporal trajectory of a hand relative to an object. In the MNS model, one region must be recognized as a hand and the other as an object with the corresponding features being based on wrist and finger positions for the hand and affordances (position of surfaces to which an action may be applied, in this case) for the object. The system then takes the trajectory of hand features as expressed in an object-centered framework, and returns a confidence level for different actions such as precision pinch and power grasp, with (in general) the confidence level for a single action rising well above the others as the action moves towards completion. However, as stressed in Section 4.1, we can recognize many actions in addition to those in our own action repertoire (Buccino et al., 2004) and link their recognition to agents, objects, events and their attributes. The visual system is thus able to bind together cognitive processes for recognizing diverse aspects of a scene and the language system can exploit (and modify) this capability to find the words and constructions to express these entities and their relationships.

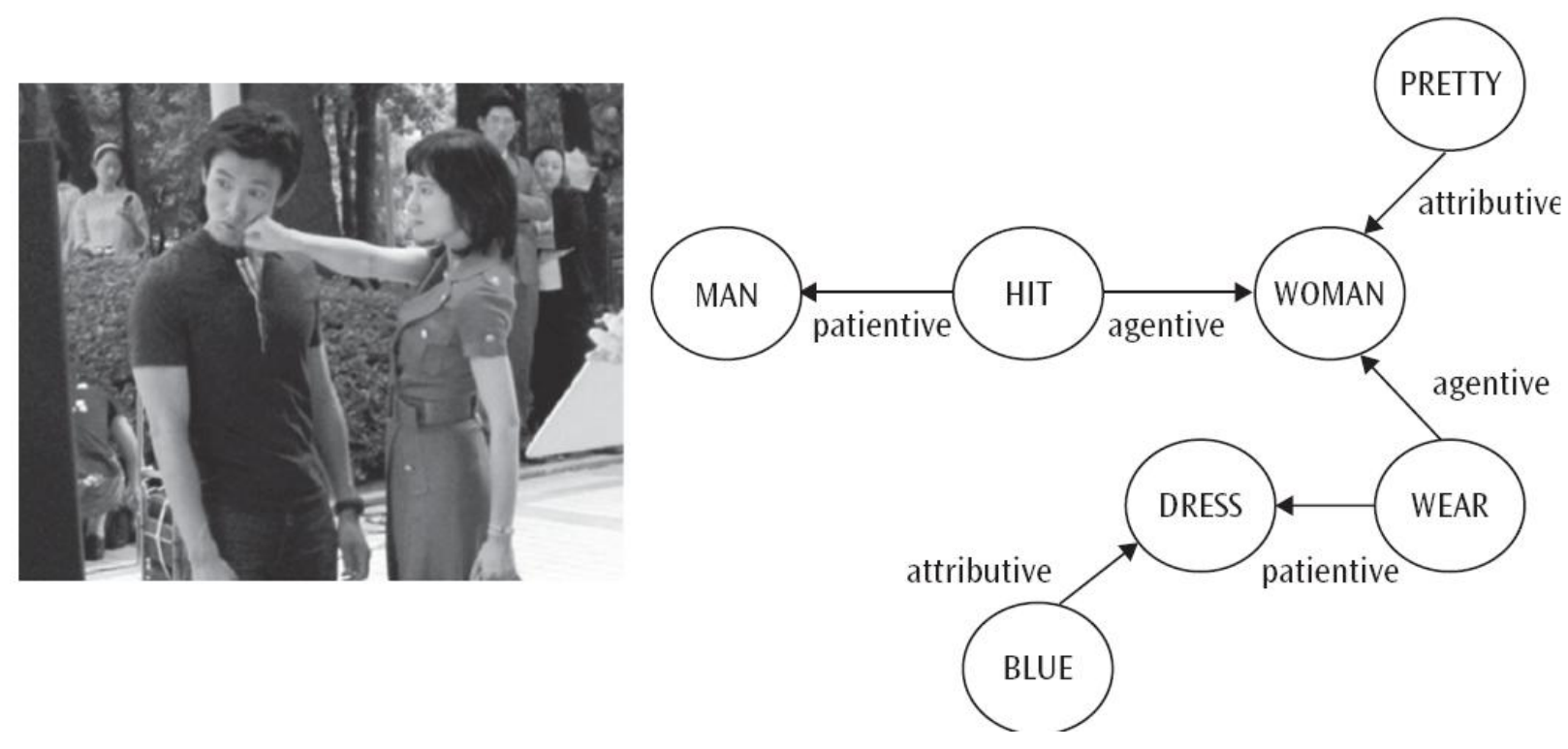

Fig. 18. Left: A picture of a woman hitting a man (original image from "Invisible Man Jangsu Choi", Korean Broadcasting System). Right: A SemRep graph that could be generated for the for the color version of the picture (where it could be seen that the woman's dress is blue).. See Arbib and Lee (2008) for a description of how Template CG may operate on this to yield the sentence "A pretty woman in blue hits a man." 
We postulate that SemRep need explicitly represent very few parameters of the observed objects and actions, and can direct requests to Visual Working Memory when the information is needed for language processing. Each parameter which does get made explicit at the SemRep level is considered an attribute and given its own node to be linked to the node for the parameterized schema. (See Arbib \& Lee, 2008, for examples and further discussion.) Consider the specific scene and SemRep shown in Fig. 18. The visual system may initially recognize a variety of aspects of the scene centered around the central figures of the man and woman, while ignoring other aspects of the scene. This analysis may combine activation of a number of schema instances together with activity in the database holding the intermediate visual representation (Section 1.3) that could be used to support further schema analysis, but has not yet done so. SemRep then abstracts from this pattern of schema activation a set of nodes and relations which constitute one possible semantic structure for the current scene - a given scene may be perceived in many different ways. The properties of a recognized object are attached to the node for that instance of the object, and the semantics of an action are attached to an edge. The attached concepts will later be translated into words by the language system. However, the SemRep graph is not labeled with words but with more abstract descriptors, allowing the same graph to be expressed in multiple ways within a given language. Thus the concept /young female/ could be translated into "girl", "woman" or even "kid" and the action concept /hitting with hand/ could be translated into "hit", "punch" or "slap". Again, the configuration where object A is placed vertically higher than $\mathrm{B}$ can be expressed as "A is above B", "B is below A", "A is on B", etc. Moreover, the same SemRep can be the basis for description in any language once the appropriate grammar and lexicon is deployed.

\section{Template Construction Grammar (TCG) and Scene Description}

We now introduce Template Construction Grammar (TCG) (Arbib \& Lee, 2008; Barrès \& Lee, 2014; Lee, 2012) to provide a visually grounded version of CG. This approach is based on the realization that our early approaches to a schema-theoretic linguistics (e.g., Arbib et al., 1987), which predated CG (Fillmore et al., 1988), can indeed be understood by interpreting the type of schemas that we then called templates as being examples of constructions as broadly construed within variants of CG.

TCG, our version of CG for language production, adopts two major policies common to all CG frameworks: (1) each construction specifies the mapping between form and meaning, and (2) 
the systematic combination of constructions yields the whole grammatical structure. But TCG also provides two key additions: (3) The semantic structure of an utterance is given as a SemRep, and (4) each construction is viewed as a schema and therefore endowed with all the dynamic properties of cooperative computation.

The lexical constructions at the bottom of Figure 19 exemplify the way in which the concept associated with a single node or edge of the SemRep can ground the selection of a word to express that concept. The constructions at the top of Figure 19 move up the hierarchy to cover larger and larger parts of the SemRep. Thus, the IN_COLOR construction has the interesting property that it must recognize the node for CLOTHING but only uses it to license the construction which yields utterances like pretty woman in blue, where the nodes for WOMAN and PRETTY have already been covered by another construction to yield the phrase pretty woman to fill the first slot in IN_COLOR, which is linked to the node HUMAN.

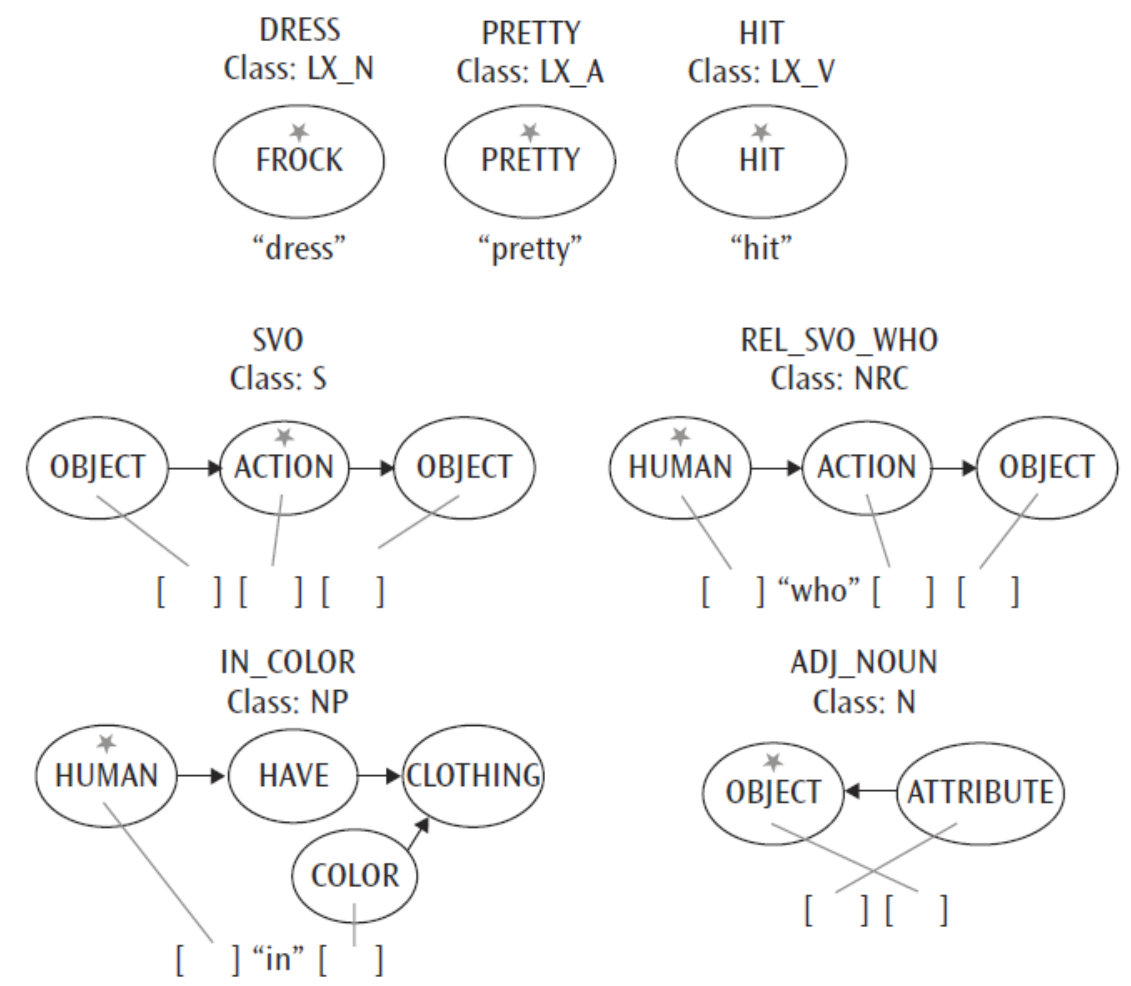

Figure 19. Examples of constructions The name and class of each construction appears above it. Each construction includes a SemRep-like graph with either generic or specific labels on the edges and nodes, with each linked to a text or an empty slot. The node labels correspond to concepts, not words. Top: Constructions that correspond to elements in the lexicon, replacing a concept (small caps) with a word or, not shown here, phrase (lower case). Bottom: Higher-level constructions used to encode grammatical information. For each slot there 
may be restrictions as to what can serve as slot fillers. The head of each construction is marked with a star (e.g. the node HUMAN in IN_COLOR). The word or phrase already associated with a node of the SemRep-like graph is then inserted into the corresponding slot of the lower-case string below the graph to yield the phrase that results from applying the construction.

During production, a SemRep may yield one or more utterances as TCG finds ways to "cover" the relevant portion of the given SemRep with a set of "small" subgraphs, where each is chosen such that there is a construction available in the language considered to express the content of that subgraph. Processing selects constructions that match whole or subparts of the SemRep graph, and can therefore participate in expressing some of its content. Thus, constructions are applied recursively, starting with lexical constructions, which have no slots (Figure 19, top), and then by applying higher-level constructions (Figure 19, bottom) as based on the results of earlier application of constructions.

In this section, we treat lexical constructions the same way we treat other constructions. However, this does not mean that lexical and more schematic constructions are neurologically equivalent. Consider cases of double dissociation in language production between anomia (patient has difficulties in naming objects or actions) and agrammatism (patient has difficulties producing syntactically correct utterances) (Goodglass, 1976).

In characterizing the cooperative computation whereby TCG produces the description of a visual scene, the processes in VISIONS are "lifted" to a similar structure (Figure 20) in which a Linguistic WM (the Working Memory for the state of applying constructions to the current SemRep) provides the workspace for the operation of construction selection and attachment, thus providing a dynamic set of hierarchically organized construction structures with varying degrees of confidence. During production of a description, a number of constructions are activated simultaneously to build upon the unfolding SemRep. Constructions cooperate and compete with each other in order to produce a verbal description of a scene. The language system, which uses the linguistic working memory, applies constructions on SemRep hierarchically and reads off the resulting formed sentence or sentence fragments that result. The vision system concurrently interprets the scene and updates the SemRep. 


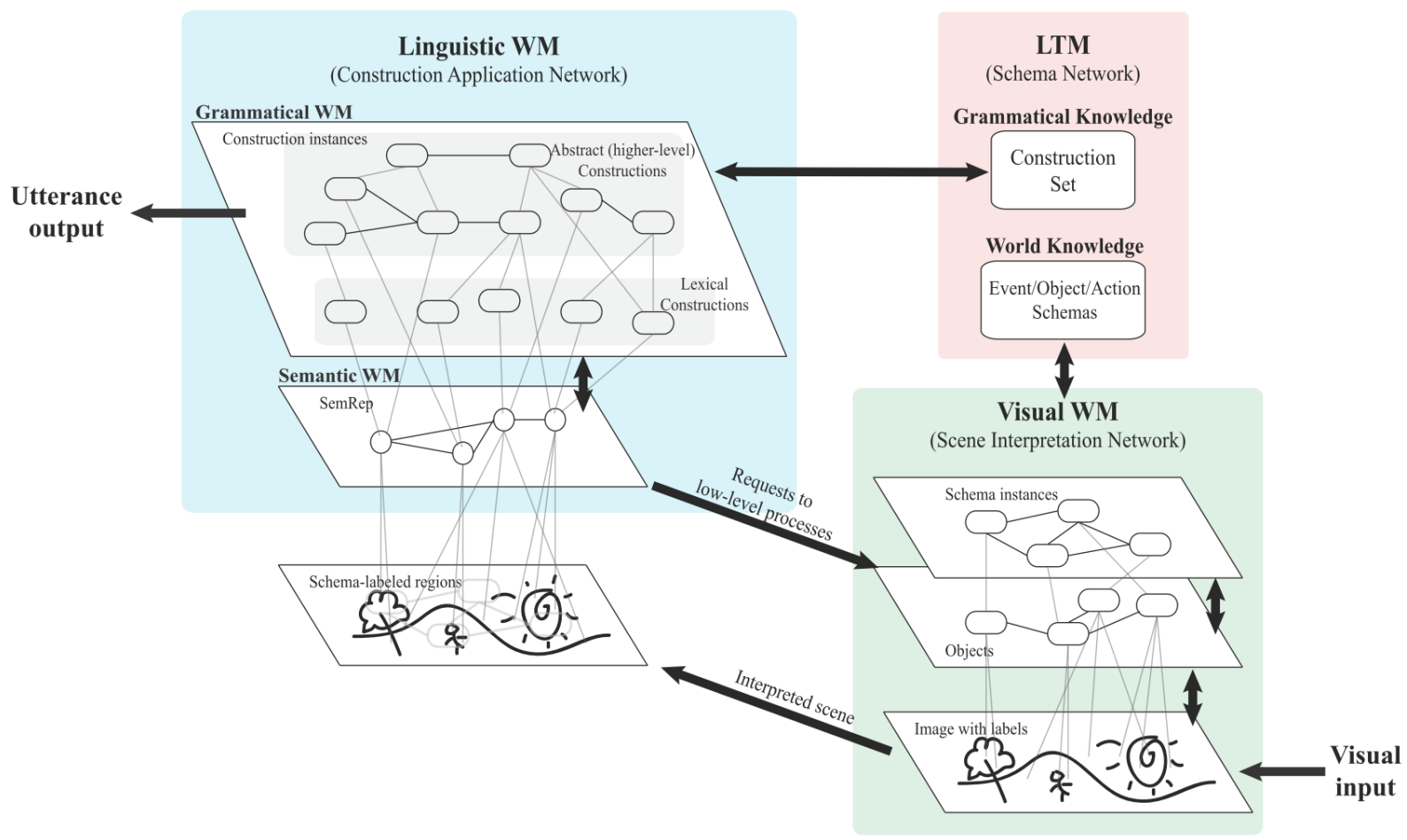

Figure 20. The structure of the SemRep/TCG model of scene description echoes the interaction of Visual WM and Long Term Memory (LTM) in VISIONS (see Figure 3), but incorporates the language system by adding a Linguistic WM and Grammatical knowledge (a set of constructions). Based on visual attention and communicative goals, the system extracts a SemRep in Semantic WM from the perceptual schemas active in Visual WM to capture key entities and relations from the scene, thus discarding many details from visual perception less relevant for verbal description. The Linguistic WM holds a hierarchical covering of the current SemRep by iterated applications of constructions from Long Term Memory. Lexical constructions are applied to associate nodes of SemRep with words, and higher-level constructions are applied to build either directly on nodes in SemRep or on an already partially completed construction assemblage. Linguistic WM provides not only a Working Memory for construction applications but also allows an utterance to be read off at any time. Just as VISIONS allows Visual WM to request more data from low-level processes, our model allows the SemRep to be updated by requesting information from the vision system when completion of an utterance requires further attention to the visual scene.

We start here with visual analysis of a scene from which a semantic representation in the form of SemRep is extracted and see how TCG may apply various constructions to yield the description of a visual scene. Conversely, as we see in the next subsection, TCG may start with an utterance and seek to find constructions and/or exploit world knowledge to find a SemRep which provides a "good enough" interpretation of the sentence. 


\section{Towards TCG as a Model of Comprehension}

Barrès and Lee (2014) proposed a conceptual extension of TCG as a model of language comprehension. The goal is two-fold: (1) to incorporate the role that world knowledge plays in the incremental generation of semantic representations during comprehension, and (2) to do so in a way that accounts for neuropsychological data on comprehension performance in aphasic patients who display certain forms of agrammatism. They focus on the performances of agrammatic aphasics in sentence-picture matching tasks during which the patient is asked to decide whether a sentence he hears matches a visual scene.

Agrammatic aphasics are patients suffering from brain lesions that result in the deterioration of their capacity to speak in a grammatically correct fashion. In contrast to a relatively unimpaired capacity to use the correct content words, agrammatic aphasics tend to omit function words, verbal inflections, etc. Caramazza and Zurif (1976) were among the first to show that agrammatic aphasics could also be impaired in their capacity to make use of syntactic cues during language comprehension. They were no different than normal subjects when asked to match a picture with canonical active sentences such as "the lion is chasing the fat tiger", but were no better than chance for center-embedded object relatives such as "the tiger that the lion is chasing is fat" However, their performance was restored to the level of normal subjects for object relatives when world knowledge cues were available to constrain the sentence interpretation as in "The apple that the boy is eating is red". This latter result led the authors to hypothesize a dissociation between a "heuristic" system based primarily on world knowledge information and an "algorithmic" system relying mainly on syntactic information. Later studies show that the situation is more complex but we ignore these complexities in this review - they remain a challenge for further research. Instead, we examine the schema-based model developed by Barrès and Lee (2014). Their focus on TCG as the language-vision interface offers a platform well suited to simulate sentence-picture matching tasks. They adopt a two-route approach to comprehension, with a world knowledge route that may be left more or less intact while lesions are performed on a grammatical route. But first we must distinguish between the roles of two different types of semantic constraints.

The core tenet of CG is that syntax and semantics are not dissociated into two different theoretical components (Croft \& Cruse, 2005). But the empirical results outlined above demand that we distinguish the world knowledge preserved in agrammatic aphasics from construction- 
related semantic constraints. We thus coin the terms heavy semantics and light semantics for world knowledge and construction-based semantics, respectively. World knowledge represents a source of information that plays a pervasive role in both visual scene analysis and language comprehension (and in action more generally) and is heavy in terms of content since it spans motor and perceptual schemas as well as abstract knowledge that we can acquire through the very use of language. Such knowledge concerning agents, objects, actions and more abstract entities contrasts with the light semantic content of constructions which develops through experiences of patterns of language describing agents, objects, actions and more. The latter may vary from the highly abstract (as in nouns versus verbs providing a language-dependent syntactic elaboration of the semantic categories of objects versus actions) or strongly linked to sensory or motor experience as illustrated by the example of the IN-COLOR construction. For us, light semantics reflects this construction-related categorization, more or less abstracted from world knowledge in a usage-based language-laden way. It is "light" because only a few semantic features matter, and it cannot be refined and enriched by interacting with the world beyond the bounds set by a given language (although of course performances vary).

David Kemmerer (2000) reported a word-picture matching task that required discrimination between 3 verbs that differed only on the basis of semantic features relevant from a grammatical point of view such as "spill”, "pour", and "sprinkle." One subject performed poorly on this wordpicture matching task while performing well in a grammaticality judgment task involving the same verbs "slotted" into constructions that matched or not in terms of the "grammatical" semantic constraints e.g., "Sam spilled beer on his pants" vs. *"Sam spilled his pants with beer." Two other patients showed the opposite pattern of performance. Such empirical results underline the need for modeling to account for the possibility of selective impairments of heavy and light semantics in language comprehension.

We now outline how TCG provides a conceptual (not yet implemented) model for the integration of light and heavy semantics underlying the dynamic interactions of world knowledge, linguistic, and visual information during language comprehension. In this case, a SemRep now serves as the output of the comprehension system and can thus serve as the basis for choosing which of several pictures corresponds to the given sentence. Crucially, the final SemRep can emerge through cooperative computation from both linguistic information and 
world knowledge. The general architecture of the TCG comprehension model is described in Figure 21.

In the work on scene description, the SemRep was generated and dynamically updated by the visual system, with construction assemblages controlling the flexible generation of utterances corresponding to all or part of the SemRep. The TCG comprehension model complements this dynamics by allowing the SemRep to be built and updated not only by the vision system, but also by two routes processing input utterances in parallel. These two routes, shown in Figure 21, are the heavy semantic route (HS) that generates semantic representations from content words using world knowledge and the grammatical route $(\mathrm{G})$ that puts grammatical knowledge to work, using grammatical cues and constraints (including light semantics) to map the surface content of the utterance input to a SemRep graph representing its semantic content. Therefore, the SemRep, during comprehension, becomes the locus of interaction between three sources of information vision, grammar, and world knowledge - that can enter into cooperative computation.

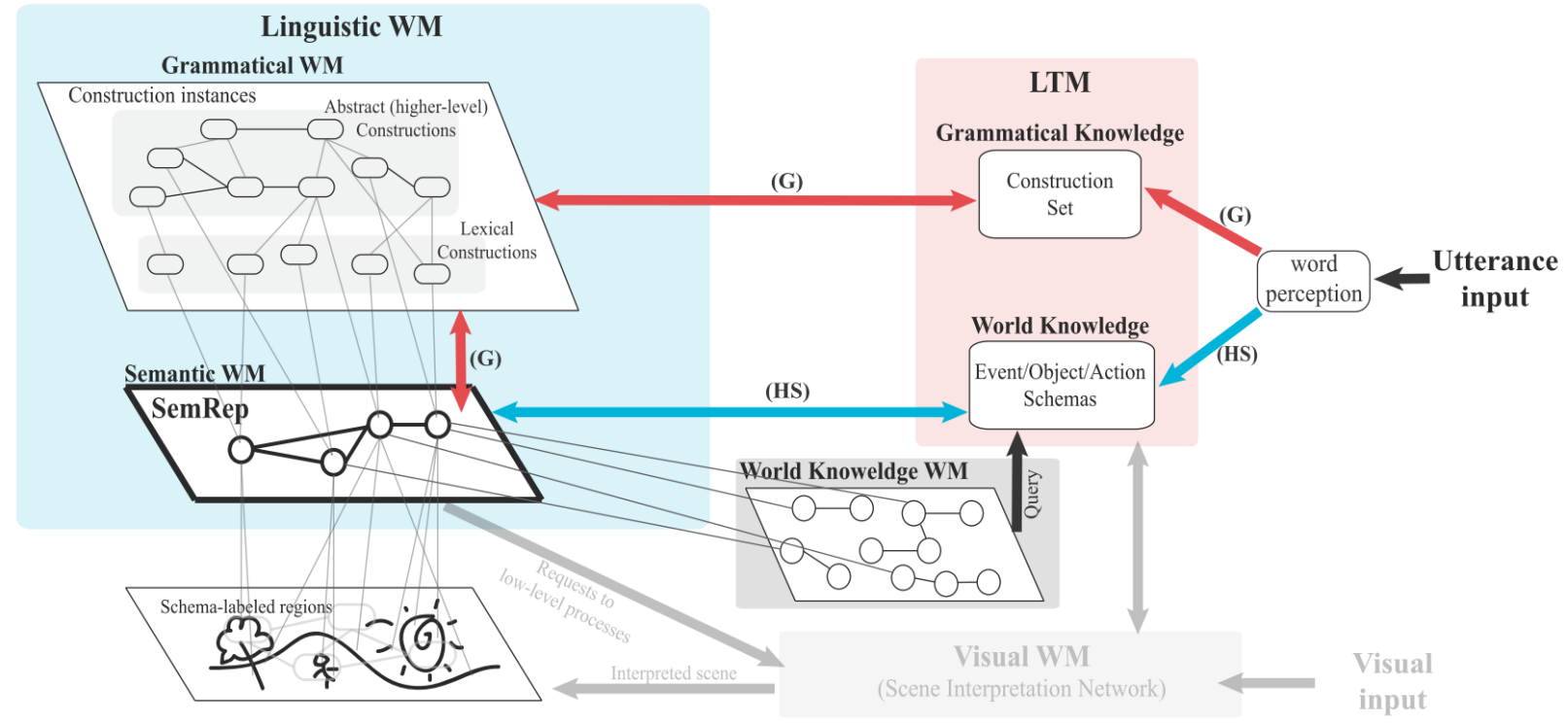

Figure 21. TCG as employed in a two-route model of language comprehension. The utterance input is fed in parallel to a grammatical route $(\mathrm{G})$ that updates the SemRep indirectly through the creation of a construction schema assemblage in grammatical working memory and to a heavy semantic route (HS) that can generate SemRep nodes directly for content words but is not sensitive to grammatical cues. In addition, the heavy semantic route incorporates the possibility for the Semantic WM to query the world knowledge WM to generate hypotheses about the plausible relations between SemRep nodes. The currently relevant hypotheses are kept active in the World Knowledge WM where they cooperate and compete to update the SemRep. Thus the Semantic WM becomes the locus of cooperative competition in which the construction assemblage on the one 
hand and the world knowledge hypotheses on the other compete and cooperate at each time to update the SemRep. The Visual working memory remains a source of input for the SemRep as in the production model.

A key feature of the TCG comprehension model's architecture lies in the fact that each input word will have an effect on two different routes triggering two parallel processes to update the SemRep. We outline here how an input word $\mathrm{W}$ is processed by these two routes:

Heavy semantic route: If $\mathrm{W}$ is a content word, the heavy semantic route will allow $\mathrm{W}$ to create or verify an existing node in Semantic Working Memory by directly accessing its associated world knowledge content representation in LTM. If W is a function word, it is ignored by the heavy semantic route that is blind to grammatical cues. Complementary to this data-driven instantiation of world knowledge, the heavy semantic route allows active SemRep nodes to query the world knowledge through a World Knowledge WM that builds plausible semantic relations between the nodes. For example, the word "eat" might not only instantiate a node for the concept EAT but also link it to a node for SOMETHING-EDIBLE. This world knowledge-based hypothesis can then verify, modify, or enrich the structure (nodes and edges) of the SemRep subgraphs currently active in Semantic WM or yield nodes which compete for later resolution. Working incrementally, upon receiving $\mathrm{W}$ the heavy semantics route updates the state of the Semantic WM, which is composed of a cloud of competing and cooperating SemRep subgraphs on which the next word W' will be received, generating anticipations of what semantic content W' will bring.

Grammatical route: Whether $\mathrm{W}$ is a content or a function word, it will result in the instantiation or verification of a construction, modifying the state of the construction assemblage in Grammatical Working Memory. In our previous production model, constructions are hierarchically applied in Linguistic WM; this can be done with varying levels of confidence (competition and cooperation) with the final utterance read out from construction assemblage that wins the competition. During comprehension, in contrast, the constructions are initially anchored on input words. At each time step, the unification of the SemRep-like subgraphs for the winning construction assemblage provides the (possibly still growing) SemRep yielding the current meaning associated with the word sequence received. Therefore, through the modification of the construction assemblage, the input $\mathrm{W}$ to the grammatical route will verify, update, or modify the SemRep graph structures in semantic working memory. By updating of the state of the semantic working memory upon receiving W, the Grammatical route, like the Heavy 
Semantics route, modifies the semantic context on which the next word W' will be received and generates anticipations. For the Grammatical route, such anticipations and context modification also extend to the Grammatical working memory: the updated construction assemblage following the reception of $\mathrm{W}$ generates new grammatical expectations for $\mathrm{W}$ '.

Following the principle of cooperative computation, the SemReps graphs active in semantic working memory are also defined both in terms of their structure and in terms of the activation levels of their components (nodes and edges), activation levels that reflect the degree of confidence associated with a relation (edges) or with some semantic content (node). Any modification made by either the heavy semantics or the grammatical route on a SemRep subgraph is expressed in terms of a change in activation levels (that can result in piece of graph being discarded altogether if its activation level becomes too low). These can therefore register the competition and cooperation of both routes.

The model of Figure 21 can handle both normal and agrammatic comprehension performances for sentence-picture matching tasks used to test agrammatic comprehension (Sherman \& Schweickert, 1989). The behavioral result of the sentence-picture matching trial rests on the complex interactions of three sources of information at the level of the SemRep. A bias towards one source of information or another can tip the cooperative computation in favor of one of the possible interpretations of the linguistic input. Discounting perceptual information while boosting the role of heavy semantics to compensate for the degradation of grammatical processing simulates the role world knowledge plays in agrammatic comprehension. However, the model puts at the forefront the fact that during a sentence-picture matching task, the impact of the perceptual content of such an image on the language comprehension system cannot be fully dissociated from that of the linguistic and heavy semantic content. Barrès and Lee (2014) develop this material in greater detail, and also explore the challenges for neuroinformatics of linking the data and modeling required to advance our understanding of the language-ready brain.

With this, we complete our computational / cognitive account of language processing and its relation to visual scene perception. The linkage to the brain remains a challenge for future research - building from the limited discussion here of possible links to agrammatism. 


\section{Challenges for Further Research}

Looking back over Sections 4.1, 4.2 and 4.3, we see that many issues remain open in defining the (not just linguistic) processing of the language-ready brain (with several of them receiving current attention):

For $\mathrm{B} \& \mathrm{~S}$, the AE-schemas are viewed as purely linguistic constructs, living within the ventral auditory stream. An interesting exercise is to see whether the constructions used in TCG can indeed be partitioned into (cooperative) processes in the dorsal and ventral streams. However, even if this were accomplished it appears that the dorsal component of the TCG-schemas would differ in several ways from AE-schemas, most specifically in their binding to SemReps and to world knowledge. But the nature of this binding is still poorly understood.

TCG would be enriched by a deeper understanding of the role of audition in two ways. One is that our knowledge of the world is based on multi-modal sensory experience during interaction with the environment, and audition (along with motor control and other sensory channels) plays a vital role. Indeed, audition and vision evolved to work together in guiding our interaction with the environment long before there was language (recall the audio-visual mirror neurons in the monkey, Keysers et al., 2003). The other is that although hearing provides the primary input for our comprehension of speech, language comprehension can also be attained via visual pathways in the case of reading (building atop auditory language) and sign language (which makes no appeal to audition). Moreover, in our perception of another's speech, we integrate the visual cues of cospeech gestures with the auditory cues. Thus, we must make an effort, extending that of B\&S (and many others, e.g., Hickok, Houde, \& Rong, 2011) to understand how early stages of visual processing of language input are executed in the brain. Note that TCG does not address this issue, even though MSH outlines an evolutionary path from action recognition to pantomime and gesture recognition and thence to [sign] language. It is well known that brain lesions can dissociate pantomime and the use of signs (Corina et al., 1992; Marshall, Atkinson, Smulovitch, Thacker, \& Woll, 2004).

We can then further ask: How do the visual and auditory streams interact when language is conveyed both audially and visually? Going further, what is mediated by the interaction of different subsystems in the brain, and to what extent are the processes supramodal, exploiting shared representations of, e.g., words that do not reflect their sensory origin (beyond the fact that 
top-down processes can interrogate upstream representations when, e.g., disambiguation of what is in the original signal is required).

The B\&S model does not address the key issue that motivates the TCG model (or other models addressed in Section 1.7 - an integrated account of these diverse CG models is a focus of current research): Language is not an isolated faculty - although we can read and write about matters abstracted from our current surroundings, a major use of language is to conduct conversations with our fellow humans that relate to the current environment. Section 4.3 offers one account of how that might occur by looking at the role of vision in analyzing a current image or scene. It is a continuing challenge for future development of MSH to put the similarities and differences in evolutionary perspective, but the achievement of TCG is instead to offer an explicit account of the brain processes necessary to address the visual world paradigm. We reiterate the need to distinguish the varied roles for vision: (i) the role of vision in recognizing manual gestures, postulated by MSH as the scaffolding for the emergence of learnable vocal gesturing and its linkage to meaning (and here we have noted the further distinction of recognizing pantomime from recognizing the signs of a language system); and (ii) the role of vision in perceiving events in the outside world about which one may want to communicate.

A major lacuna in the models of Section 4 is that they focus on comprehension and production one-at-a time. A major challenge, then, is to give far more attention (back to the dyad) to the semiotic cycle of Figure 2 (a key issue in the FCG modeling of language games) and to dyadic brain modeling (which was at the heart of our discussion of the emergence of ape gesture in Section 2.2) - see Jeannerod (2005) for a related account of human interaction. We may note here the concern of Garrod and Pickering for the processes involved in human conversation (Garrod \& Pickering, 2004, 2009; Pickering \& Garrod, 2013). Relevant neural data are now available (Menenti, Garrod, \& Pickering, 2012; Menenti, Gierhan, Segaert, \& Hagoort, 2011; Segaert, Menenti, Weber, Petersson, \& Hagoort, 2012) but their interpretation remains somewhat problematic.

Although the modern human brain's capacity for language has dominated the discussion of Section 4, this was part of an attempt (still very much in progress) to define what it is that evolved in the evolution of the human language-ready brain. Section 2 focused on hypotheses on the evolution of the language-ready brain from the brains of LCA-m and LCA-c; Section 3 
assessed how cultural evolution might have led ancestors equipped with language-ready brains to develop language across (perhaps) a thousand millennia.

Sections 1 and 2 introduced comparative neuroprimatology as an umbrella for this work, embracing multiple, distinct strands of empirical research including:

- studying the relation between neuroanatomy and neurophysiology in exploring the neural circuitry underlying the linkage of vision and manual action in the macaque;

- the use of fieldwork in studying the relation between behavior and gesture in apes, and the use of diffusion tensor imaging (we cited Hecht et al., 2012; but note also the work of Rilling, 2014; J.K. Rilling et al., 2008), not neurophysiology, to offer clues for comparison of chimpanzee brains with those of macaques and humans; and

- the use of studies linking vision and language (the visual world paradigm), as well as lesion studies of agrammatism and (although we had little to say about this) brain imaging studies in neurolinguistics.

Clearly, each subarea listed here can provide scope for a whole career of digging deeply, whether or not one is concerned with evolutionary questions, and clearly a concern with evolution brings other disciplines into play, including studies of genomics and niche construction.

At the same time, we extended the scope of our investigation to computational comparative neuroprimatology employing both neural network modeling and schema-theoretic approaches, and suggested a methodology (Figure 4) whereby insights into the details of macaque circuitry might be used in concert with (and in testing of) evolutionary hypotheses to derive computational models of the circuitry of the brains of apes and humans that cannot be tested against direct evidence on the cell-by-cell neurophysiology of those brains. Throughout, we tried not only to demonstrate models which offered steps forward in our understanding but to make clear that each success in turn opened up new challenges for further turns of the experiment-modeling cycle, as each model poses new challenges for empirical research, and each new set of interlinked data challenges us to question our existing models and, as appropriate, modify them or replace them.

Acknowledgment: This material is based in part on work supported by the National Science Foundation under Grant No. 0924674 and Grant No. BCS-1343544 "INSPIRE Track 1: Action, Vision and Language, and their Brain Mechanisms in Evolutionary Relationship" (Michael A. 
Arbib, Principal Investigator). A textbook version of the first draft of this review appears as Chapter 25 of From Neuron to Cognition via Computational Neuroscience (Arbib \& Bonaiuto, 2016). I thank all my colleagues whose work is reported here, but especially Victor Barrès for detailed discussion of Sections 4.2 and 4.3.

\section{References}

Aboitiz, F. (1995). Working memory networks and the origin of language areas in the human brain. Med Hypotheses, 44(6), 504-506.

Aboitiz, F. (2012). Gestures, vocalizations and memory in language origins. [Review]. Frontiers in Evolutionary Neuroscience, 4(2), doi: 10.3389/fnevo.2012.00002. doi: 10.3389/fnevo.2012.00002

Aboitiz, F. (2013). How did vocal behavior "take over" the gestural communication system? Language and Cognition, 5, $167-176$.

Aboitiz, F., Aboitiz, S., \& García, R. (2010a). The phonological loop: A key innovation in human evolution. Current Anthropology, 51, S55-S65.

Aboitiz, F., Aboitiz, S., \& García, R. R. (2010b). The Phonological Loop: A Key Innovation in Human Evolution. Current Anthropology, 51(1), S55-S65.

Aboitiz, F., \& García, R. (2009). Merging of Phonological and Gestural Circuits in Early Language Evolution. Reviews in the Neurosciences, 20(1), 71-84.

Aboitiz, F., Garcia, R. R., Bosman, C., \& Brunetti, E. (2006). Cortical memory mechanisms and language origins. Brain Lang, 98(1), 40-56.

Aboitiz, F., \& Garcia, V. R. (1997). The evolutionary origin of the language areas in the human brain. A neuroanatomical perspective. Brain Res Brain Res Rev, 25(3), 381-396.

Alstermark, B., Lundberg, A., Norrsell, U., \& Sybirska, E. (1981). Integration in descending motor pathways controlling the forelimb in the cat: 9. Differential behavioural defects after spinal cord lesions interrupting defined pathways from higher centres to motoneurones. Experimental Brain Research, 42, 299-318.

Altmann, G. T. M., \& Kamide, Y. (1999). Incremental interpretation at verbs: restricting the domain of subsequent reference. Cognition, 73(3), 247-264. doi: 10.1016/s00100277(99)00059-1

Altmann, G. T. M., \& Kamide, Y. (2009). Discourse-mediation of the mapping between language and the visual world: Eye movements and mental representation. Cognition, 111(1), 55-71. doi: 10.1016/j.cognition.2008.12.005

Arbib, M. A. (1981). Perceptual structures and distributed motor control. In V. B. Brooks (Ed.), Handbook of Physiology - The Nervous System II. Motor Control (pp. 1449-1480). Bethesda, MD: American Physiological Society.

Arbib, M. A. (1988). Schemas and Neurons: Two Levels of Neural Computing. In R. Eckmiller \& C. v. d. Malsburg (Eds.), Neural Computing (pp. 331-320): Springer-Verlag.

Arbib, M. A. (1989). The Metaphorical Brain 2: Neural Networks and Beyond. New York: Wiley-Interscience.

Arbib, M. A. (2002). The mirror system, imitation, and the evolution of language. In K. Dautenhahn \& C. L. Nehaniv (Eds.), Imitation in Animals and Artifacts. Complex Adaptive Systems (pp. 229-280). Cambridge, MA: The MIT Press. 
Arbib, M. A. (2005). From monkey-like action recognition to human language: an evolutionary framework for neurolinguistics. The Behavioral and brain sciences, 28(2), 105-124; discussion 125-167-105-124; discussion 125-167.

Arbib, M. A. (2006). Aphasia, apraxia and the evolution of the language-ready brain. Aphasiology, 20, 1-30.

Arbib, M. A. (2007). How New Languages Emerge (Review of D. Lightfoot, 2006, How New Languages Emerge, Cambridge University Press). Linguist List, 18-432, Thu Feb 08 2007, http://linguistlist.org/issues/17/17-1250.html.

Arbib, M. A. (2010). Mirror System Activity for Action and Language is Embedded in the Integration of Dorsal \& Ventral Pathways. Brain and Language, 112(1), 12-24.

Arbib, M. A. (2012). How the Brain Got Language: The Mirror System Hypothesis. New York \& Oxford: Oxford University Press.

Arbib, M. A. (2013a). Complex Imitation and the Language-Ready Brain. Language and Cognition, 5(2-3).

Arbib, M. A. (2013b). Précis of How the Brain Got Language: The Mirror System Hypothesis. Language and Linguistics Compass, 5.

Arbib, M. A. (2015). Neurolinguistics. In B. Heine \& H. Narrog (Eds.), The Oxford Handbook of Linguistic Analysis, Second Edition (pp. 473-502). Oxford, New York: Oxford University Press.

Arbib, M. A., \& Bickerton, D. (Eds.). (2010). The Emergence of Protolanguage: Holophrasis vs compositionality. Philadelphia, Amsterdam: John Benjamins Publishing Company.

Arbib, M. A., Bischoff, A., Fagg, A. H., \& Grafton, S. T. (1995). Synthetic PET: analyzing large-scale properties of neural networks. Human Brain Mapping, 2(4), 225-233.

Arbib, M. A., \& Bonaiuto, J. J. (2012). Multiple Levels of Spatial Organization: World Graphs and Spatial Difference Learning. Adaptive Behavior, 287-303(4), 287-303.

Arbib, M. A., \& Bonaiuto, J. J. (Eds.). (2016). From Neuron to Cognition via Computational Neuroscience. Cambridge, MA: The MIT Press.

Arbib, M. A., \& Bota, M. (2003). Language Evolution: Neural Homologies and Neuroinformatics. Neural Networks, 16, 1237-1260.

Arbib, M. A., \& Caplan, D. (1979). Neurolinguistics must be Computational. Behavioral and Brain Sciences, 2, 449-483.

Arbib, M. A., Caplan, D., \& Marshall, J. C. (Eds.). (1982). Neural Models of Language Processes. New York: Academic Press.

Arbib, M. A., Conklin, E. J., \& Hill, J. C. (1987). From Schema Theory to Language. New York: Oxford University Press.

Arbib, M. A., Fagg, A. H., \& Grafton, S. T. (2002). Synthetic PET Imaging for Grasping: From Primate Neurophysiology to Human Behavior. In F. T. Soomer \& A. Wichert (Eds.), Exploratory Analysis and Data Modeling in Functional Neuroimaging (pp. 231-250): MIT Press.

Arbib, M. A., Ganesh, V., \& Gasser, B. (2014). Dyadic Brain Modeling, Ontogenetic Ritualization of Gesture in Apes, and the Contributions of Primate Mirror Neuron Systems. Phil Trans Roy Soc B, 369 (1644), 20130414.

Arbib, M. A., Gasser, B., \& Barrès, V. (2014). Language is handy but is it embodied? Neuropsychologia, 55, 57-70. 
Arbib, M. A., \& Hill, J. C. (1988). Language Acquisition: Schemas Replace Universal Grammar. In J. A. Hawkins (Ed.), Explaining Language Universals (pp. 56-72). Oxford: Basil Blackwell.

Arbib, M. A., \& Lee, J. Y. (2008). Describing visual scenes: Towards a neurolinguistics based on construction grammar. Brain Research, 1225, 146-162.

Arbib, M. A., Liebal, K., \& Pika, S. (2008). Primate Vocalization, Gesture, and the Evolution of Human Language. Current Anthropology, 49(6), 1053-1076. doi: doi:10.1086/593015 \%U http://www.journals.uchicago.edu/doi/abs/10.1086/593015

Arbib, M. A., \& Mundhenk, T. N. (2005). Schizophrenia and the mirror system: an essay. Neuropsychologia, 43(2), 268-280.

Arbib, M. A., \& Rizzolatti, G. (1997). Neural expectations: a possible evolutionary path from manual skills to language. Communication and Cognition, 29, 393-424.

Baddeley, A. D. (2003). Working memory: looking back and looking forward. Nat Rev Neurosci, 4(10), 829-839.

Baddeley, A. D., \& Hitch, G. J. (1974). Working memory. In G. A. Bower (Ed.), The Psychology of Learning and Motivation. New York: Academic Press.

Baggio, G., \& Hagoort, P. (2011). The balance between memory and unification in semantics: A dynamic account of the N400. Language and Cognitive Processes, 26(9), 1338-1367.

Baldwin, J. M. (1896). A new factor in evolution. American Naturalist, 30, 441-451, 536-533.

Barrès, V., \& Lee, J. Y. (2014). Template Construction Grammar: From Visual Scene Description to Language Comprehension and Agrammatism. Neuroinformatics, 12(1), 181-208. doi: 10.1007/s12021-013-9197-y

Barrès, V., Simons, A., \& Arbib, M. A. (2013). Synthetic event-related potentials: A computational bridge between neurolinguistic models and experiments. Neural Networks, 37, 66-92. doi: http://dx.doi.org/10.1016/j.neunet.2012.09.021

Bates, E., \& MacWhinney, B. (1989). Functionalism and the competition model. The crosslinguistic study of sentence processing, 3-73.

Beuls, K., \& Steels, L. (2013). Agent-Based Models of Strategies for the Emergence and Evolution of Grammatical Agreement. PLoS One, 8(3), e58960. doi: 10.1371/journal.pone.0058960

Bickel, B. (2010). Absolute and statistical universals. In P. C. Hogan (Ed.), The Cambridge encyclopedia of the language sciences (pp. 77-79). Cambridge: Cambridge University Press.

Bickerton, D. (1995). Language and Human Behavior. Seattle: University of Washington Press.

Bonaiuto, J. J., \& Arbib, M. A. (2010). Extending the mirror neuron system model, II: what did I just do? A new role for mirror neurons. [Research Support, U.S. Gov't, Non-P.H.S.]. Biol Cybern, 102(4), 341-359. doi: 10.1007/s00422-010-0371-0

Bonaiuto, J. J., Rosta, E., \& Arbib, M. A. (2007). Extending the mirror neuron system model, I : Audible actions and invisible grasps. Biol Cybern, 96, 9-38.

Bornkessel-Schlesewsky, I., \& Schlesewsky, M. (2009). The Role of Prominence Information in the Real-Time Comprehension of Transitive Constructions: A Cross-Linguistic Approach. Language and Linguistics Compass, 3, 19-58.

Bornkessel-Schlesewsky, I., \& Schlesewsky, M. (2013). Reconciling time, space and function: A new dorsal-ventral stream model of sentence comprehension. Brain Lang, 125(1), 60-76. doi: http://dx.doi.org/10.1016/j.bandl.2013.01.010 
Bornkessel, I., \& Schlesewsky, M. (2006). The extended argument dependency model: a neurocognitive approach to sentence comprehension across languages. Psychological Review, 113(4), 787-821.

Bota, M., \& Arbib, M. A. (2004). Integrating databases and expert systems for the analysis of brain structures: connections, similarities, and homologies. Neuroinformatics, 2(1), 1958.

Buccino, G., Lui, F., Canessa, N., Patteri, I., Lagravinese, G., Benuzzi, F., . . Rizzolatti, G. (2004). Neural circuits involved in the recognition of actions performed by nonconspecifics: an FMRI study. J Cogn Neurosci, 16(1), 114-126.

Bullock, D., \& Rhodes, B. J. (2003). Competitive queuing for planning and serial performance. In M. A. Arbib (Ed.), The Handbook of Brain Theory and Neural Networks, Second Edition (pp. 241-244). Cambridge, MA: A Bradford Book/The MIT Press.

Byrne, R. W. (2003). Imitation as behavior parsing. Philosophical Transactions of the Royal Society of London (B), 358, 529-536.

Byrne, R. W., \& Byrne, J. M. E. (1993). Complex leaf-gathering skills of mountain gorillas (Gorilla g. beringei): Variability and standardization. American Journal of Primatology, 31, 241-261.

Caramazza, A., \& Zurif, E. B. (1976). Dissociation of algorithmic and heuristic processes in language comprehension: Evidence from aphasia. [10.1016/0093-934X(76)90048-1]. Brain and Language, 3(4), 572-582.

Corina, D. P., Poizner, H., Bellugi, U., Feinberg, T., Dowd, D., \& O'Grady-Batch, L. (1992). Dissociation between linguistic and nonlinguistic gestural systems: a case for compositionality. Brain and Language, 43 (3), 414-447.

Cornelissen, P., Hansen, P., Kringelbach, M., \& Pugh, K. (2010). The neural basis of reading. Oxford: Oxford University Press.

Coudé, G., Ferrari, P. F., Rodà, F., Maranesi, M., Borelli, E., Veroni, V., . . . Fogassi, L. (2011). Neurons Controlling Voluntary Vocalization in the Macaque Ventral Premotor Cortex. PLoS One, 6(11), e26822. doi: 10.1371/journal.pone.0026822

Croft, W. (2001). Radical construction grammar: syntactic theory in typological perspective. Oxford: Oxford University Press.

Croft, W., \& Cruse, D. A. (2005). Cognitive Linguistics. Cambridge: Cambridge University Press.

Custance, D. M., Whiten, A., \& Bard, K. A. (1995). Can young chimpanzees (Pan troglodytes) imitate arbitrary actions? Hayes \& Hayes (1952) revisited. Behaviour, 132, 11-12.

Dehaene, S., \& Cohen, L. (2007). Cultural Recycling of Cortical Maps. Neuron, 56(2), 384-398. doi: http://dx.doi.org/10.1016/j.neuron.2007.10.004

Dehaene, S., Pegado, F., Braga, L. W., Ventura, P., Filho, G. N., Jobert, A., . . . Cohen, L. (2010). How Learning to Read Changes the Cortical Networks for Vision and Language. Science, 330(6009), 1359-1364. doi: 10.1126/science.1194140

Didday, R. L., \& Arbib, M. A. (1975). Eye movements and visual perception: 'two visual systems' model. International Journal of Man-Machine Studies, 7, 547-569.

Dominey, P. F., Arbib, M. A., \& Joseph, J.-P. (1995). A model of corticostriatal plasticity for learning oculomotor associations and sequences. Journal of Cognitive Neuroscience, 7(3), 311-336.

Dominey, P. F., \& Inui, T. (2009). Cortico-striatal function in sentence comprehension: Insights from neurophysiology and modeling. Cortex. 
Draper, B. A., Collins, R. T., Brolio, J., Hanson, A. R., \& Riseman, E. M. (1989). The schema system. International Journal of Computer Vision, 2, 209-250.

Dryer, M. S. (2011). Order of subject, object and verb (http://wals.info/chapter/81, retrieved on April 8, 2012). In M. S. Dryer \& M. Haspelmath (Eds.), The world atlas of language structures online Munich: Max Planck Digital Library.

Emmorey, K. (2002). Language, Cognition, and the Brain: Insights from Sign Language Research. Mahwah, NJ: Lawrence Erlbaum and Associates.

Evans, N., \& Levinson, S. C. (2009). The myth of language universals: Language diversity and its importance for cognitive science. Behavioral and Brain Sciences, 32(05), 429-448. doi: doi:10.1017/S0140525X0999094X

Fagg, A. H., \& Arbib, M. A. (1998). Modeling parietal-premotor interactions in primate control of grasping. Neural Netw, 11(7-8), 1277-1303.

Feldman, J., \& Narayanan, S. (2004). Embodied meaning in a neural theory of language. Brain Lang, 89(2), 385-392.

Ferrari, P. F., Gallese, V., Rizzolatti, G., \& Fogassi, L. (2003). Mirror neurons responding to the observation of ingestive and communicative mouth actions in the monkey ventral premotor cortex. Eur J Neurosci, 17(8), 1703-1714.

Fillmore, C. J., Kay, P., \& O’Connor, M. K. (1988). Regularity and idiomaticity in grammatical constructions: the case of let alone. Language \& Cognitive Processes, 64, 501-538.

Fogassi, L., Coudé, G., \& Ferrari, P. F. (2013). The extended features of mirror neurons and the voluntary control of vocalization in the pathway to language. Language and Cognition, 5, $145-155$.

Friederici, A. (2012). The cortical language circuit: from auditory perception to sentence comprehension. Trends Cogn. Neurosci., 16, 262-268.

Friederici, A. D. (2011). The brain basis of language processing: from structure to function. [10.1152/physrev.00006.2011]. Physiological Reviews, 91(4), 1357-1392.

Friederici, A. D. (2012). The cortical language circuit: from auditory perception to sentence comprehension. [10.1016/j.tics.2012.04.001]. Trends in Cognitive Sciences, 16(5), 262268.

Garrod, S., \& Pickering, M. J. (2004). Why is conversation so easy? Trends Cogn Sci, 8(1), 8-11.

Garrod, S., \& Pickering, M. J. (2009). Joint Action, Interactive Alignment, and Dialog. Topics in Cognitive Science, 1(2), 292-304. doi: 10.1111/j.1756-8765.2009.01020.x

Gasser, B., Cartmill, E., \& Arbib, M. A. (2014). Ontogenetic ritualization of primate gesture as a case study in dyadic brain modeling. Neuroinformatics, 12(1), 93-109.

Goldberg, A. E. (1995). Constructions: a Construction Grammar approach to argument structure. Chicago: The University of Chicago Press.

Goodglass, H. (1976). Agrammatism. Studies in neurolinguistics, 1, 237-260.

Grafton, S. T., Arbib, M. A., Fadiga, L., \& Rizzolatti, G. (1996). Localization of grasp representations in humans by PET: 2. Observation compared with imagination. Experimental Brain Research, 112, 103-111.

Hagoort, P. (2005). On Broca, brain, and binding: a new framework. Trends Cogn Sci, 9(9), 416423.

Hagoort, P. (2013). MUC (Memory, Unification, Control) and beyond. Frontiers in Psychology, 4. 
Hanson, A. R., \& Riseman, E. M. (1978). VISIONS: a computer system for interpreting scenes. In A. R. Hanson \& E. M. Riseman (Eds.), Computer Vision Systems (pp. 129-163). New York: Academic Press.

Haspelmath, M., Dryer, M. S., Gil, D., \& Comrie, B. (2005). The World Atlas of Language Structures. Oxford: Oxford University Press.

Hawkins, J. A. (1999). Processing Complexity and Filler-Gap Dependencies across Grammars. Language \& Cognitive Processes, 75(2), 244-285.

Hecht, E. E., Gutman, D. A., Preuss, T. M., Sanchez, M. M., Parr, L. A., \& Rilling, J. K. (2012). Process Versus Product in Social Learning: Comparative Diffusion Tensor Imaging of Neural Systems for Action Execution-Observation Matching in Macaques, Chimpanzees, and Humans. Cerebral Cortex. doi: 10.1093/cercor/bhs097

Heine, B., \& Kuteva, T. (2011). Grammaticalization theory as a tool for reconstructing language evolution. In M. Tallerman \& K. Gibson (Eds.), The Oxford Handbook of Language Evolution (pp. in press). Oxford: Oxford University Press.

Hickok, G. (2012). Computational neuroanatomy of speech production. Nature Rev. Neurosci., $13,135-145$.

Hickok, G., Houde, J., \& Rong, F. (2011). Sensorimotor integration in speech processing: Computational basis and neural organization. Neuron, 69, 407-422.

Hickok, G., \& Poeppel, D. (2004). Dorsal and ventral streams: a framework for understanding aspects of the functional anatomy of language. [10.1016/j.cognition.2003.10.011]. Cognition, 92(1-2), 67-99.

Hill, J. C. (1983). A computational model of language acquisition in the two-year-old. Cognition and Brain Theory, 6, 287-317.

Hobaiter, C., \& Byrne, R. W. (2011). The gestural repertoire of the wild chimpanzee. Animal cognition, 14, 745-767. doi: 10.1007/s10071-011-0409-2

Houghton, G., \& Hartley, T. (1995). Parallel Models of Serial Behavior: Lashley Revisited. Psyche, 2(25).

Hubel, D. H., \& Wiesel, T. N. (1977). Ferrier lecture: Functional architecture of macaque monkey visual cortex. Proceedings of the Royal Society of London. Series B, Biological Sciences, 1-59.

Hurford, J. R. (2011). The Origins of Grammar II: Language in the Light of Evolution. Oxford, New York: Oxford University Press.

Iriki, A., \& Taoka, M. (2012). Triadic (ecological, neural, cognitive) niche construction: a scenario of human brain evolution extrapolating tool use and language from the control of reaching actions. Philosophical Transactions of the Royal Society B: Biological Sciences, 367, 10-23

Itti, L., \& Koch, C. (2000). A saliency-based search mechanism for overt and covert shifts of visual attention. Vision Research, 40, 1489-1506.

Jakobson, L. S., Archibald, Y. M., Carey, D. P., \& Goodale, M. A. (1991). A kinematic analysis of reaching and grasping movements in a patient recovering from optic ataxia. Neuropsychologia, 29, 803-809.

Jeannerod, M. (2005). How do we decipher others' minds? In J.-M. Fellous \& M. A. Arbib (Eds.), Who Needs Emotions: The Brain Meets the Robot (pp. 147-169). Oxford, New York: Oxford University Press. 
Jeannerod, M., \& Biguer, B. (1982). Visuomotor mechanisms in reaching within extra-personal space. In D. J. Ingle, R. J. W. Mansfield \& M. A. Goodale (Eds.), Advances in the Analysis of Visual Behavior (pp. 387-409). Cambridge, MA: The MIT Press.

Jeannerod, M., Decety, J., \& Michel, F. (1994). Impairment of grasping movements following a bilateral posterior parietal lesion. Neuropsychologia, 32(4), 369-380.

Kemmerer, D. (2000). Grammatically relevant and grammatically irrelevant features of verb meaning can be independently impaired. [10.1080/02687030050156584]. Aphasiology, 14(10), 997-1020.

Kemmerer, D. (2012). The Cross-Linguistic Prevalence of SOV and SVO Word Orders Reflects the Sequential and Hierarchical Representation of Action in Broca's Area. Language and Linguistics Compass, 6(1), 50-66. doi: 10.1002/lnc3.322

Kemmerer, D. (2015). Cognitive Neuroscience of Language. New York and London: Psychology Press, Taylor \& Francis Group.

Kendon, A. (2004). Gesture: Visible Action as Utterance. Cambridge: Cambridge University Press.

Keysers, C., Kohler, E., Umiltà, M. A., Nanetti, L., Fogassi, L., \& Gallese, V. (2003). Audiovisual mirror neurons and action recognition. Exp Brain Res, 153(4), 628-636.

Knoeferle, P., \& Crocker, M. W. (2006). The Coordinated Interplay of Scene, Utterance, and World Knowledge: Evidence From Eye Tracking. Cognitive Science, 30 481-529.

Koch, C., \& Ullman, S. (1985). Shifts in selective visual attention: towards the underlying neural circuitry. Human Neurobiology, 4, 219-227.

Koechlin, E., \& Jubault, T. (2006). Broca's Area and the Hierarchical Organization of Human Behavior. Neuron, 50(6), 963-974.

Lee, J. Y. (2012). Linking Eyes to Mouth: A Schema-based Computational Model for Describing Visual Scenes. Ph.D. Thesis, Computer Science, University of Southern California, Los Angeles, CA.

Liberman, A. M., \& Mattingly, I. G. (1985). The motor theory of speech perception revised. Cognition, 21, 1-36.

Lightfoot, D. W. (2006). How New Languages Emerge. Cambridge: Cambridge University Press.

Lotto, A. J., Hickok, G. S., \& Holt, L. L. (2009). Reflections on mirror neurons and speech perception. Trends Cogn Sci, 13(3), 110-114.

MacWhinney, B. (2005). A unified model of language development. In J. F. Kroll \& A. M. B. D. Groot (Eds.), Handbook of bilingualism: Psycholinguistic approaches (pp. 49-67). Oxford: Oxford University Press.

Marshall, J., Atkinson, J., Smulovitch, E., Thacker, A., \& Woll, B. (2004). Aphasia in a user of British Sign Language: Dissociation between sign and gesture. Cognitive Neuropsychology, 21, 537-554.

McNeill, D. (2005). Gesture and Thought. Chicago: University of Chicago Press.

Menenti, L., Garrod, S. C., \& Pickering, M. J. (2012). Towards a neural basis of interactive alignment in conversation. [Review]. Frontiers in Human Neuroscience, 6, 0.3389/fnhum.2012.00185. doi: 10.3389/fnhum.2012.00185

Menenti, L., Gierhan, S. M. E., Segaert, K., \& Hagoort, P. (2011). Shared Language Overlap and Segregation of the Neuronal Infrastructure for Speaking and Listening Revealed by Functional MRI. Psychological Science, 22(9), 1173-1182. doi: $10.1177 / 0956797611418347$ 
Moulin-Frier, C., \& Arbib, M. A. (2013). Recognizing Speech in a Novel Accent: The Motor Theory of Speech Perception Reframed. Submitted for publication, Submitted.

Narayanan, S. S. (1997). Knowledge-based Action Representations for Metaphor and Aspect (KARMA). (Ph.D.), University of California at Berkeley.

Novick, J. M., Trueswell, J. C., \& Thompson-Schill, S. L. (2005). Cognitive control and parsing: Reexamining the role of Broca's area in sentence comprehension. Cognitive, Affective, \& Behavioral Neuroscience, 5(3), 263-281. doi: 10.3758/cabn.5.3.263

Odling-Smee, F. J., Laland, K. N., \& Feldman, M. W. (2003). Niche construction: The neglected process in evolution. Princeton, NJ: Princeton University Press.

Oztop, E., \& Arbib, M. A. (2002). Schema design and implementation of the grasp-related mirror neuron system. Biol Cybern, 87(2), 116-140.

Oztop, E., Kawato, M., \& Arbib, M. A. (2013). Mirror neurons: Functions, mechanisms and models. Neuroscience Letters, $\quad 540 \quad 43-55 . \quad$ doi: http://dx.doi.org/10.1016/j.neulet.2012.10.005

Petersson, K. M., Reis, A., Askelof, S., Castro-Caldas, A., \& Ingvar, M. (2000). Language processing modulated by literacy: a network analysis of verbal repetition in literate and illiterate subjects. J Cogn Neurosci, 12(3), 364-382.

Pickering, M. J., \& Garrod, S. (2013). An Integrated Theory of Language Production and Comprehension. Behavioral and Brain Sciences, 36(4), 329-347.

Poeppel, D., Emmorey, K., Hickok, G., \& Pylkkänen, L. (2012). Towards a New Neurobiology of Language. The Journal of Neuroscience, 32(41), 14125-14131. doi: 10.1523/jneurosci.3244-12.2012

Poizner, H., Klima, E., \& Bellugi, U. (1987). What the hands reveal about the brain. Cambridge, MA: MIT Press.

Rauschecker, J. P. (1998). Cortical processing of complex sounds. Curr Opin Neurobiol, 8(4), 516-521.

Rauschecker, J. P. (2005). Vocal gestures and auditory objects. Behavioral and Brain Sciences, 28(02), 143-144. doi: doi:10.1017/S0140525X05410031

Rauschecker, J. P. (2011). An expanded role for the dorsal auditory pathway in sensorimotor control and integration. [10.1016/j.heares.2010.09.001]. Hearing Research, 271(1-2), 1625.

Rauschecker, J. P., \& Scott, S. K. (2009). Maps and streams in the auditory cortex: nonhuman primates illuminate human speech processing. [10.1038/nn.2331]. Nature neuroscience, 12(6), 718-724.

Rauschecker, J. P., \& Tian, B. (2000). Mechanisms and streams for processing of "what" and "where" in auditory cortex. Proceedings of the National Academy of Sciences, 97(22), 11800-11806. doi: 10.1073/pnas.97.22.11800

Rilling, J. K. (2014). Comparative primate neurobiology and the evolution of brain language systems. Current Opinion in Neurobiology, 28(0), 10-14. doi: http://dx.doi.org/10.1016/j.conb.2014.04.002

Rilling, J. K., Glasser, M. F., Preuss, T. M., Ma, X., Zhao, T., Hu, X., \& Behrens, T. E. (2008). The evolution of the arcuate fasciculus revealed with comparative DTI. Nature Neuroscience, 11(4), 426-428.

Rilling, J. K., Glasser, M. F., Preuss, T. M., Ma, X., Zhao, T., Hu, X., \& Behrens, T. E. J. (2008). The evolution of the arcuate fasciculus revealed with comparative DTI. Nature Neuroscience, 11(4), 426-428. 
Rizzolatti, G., \& Arbib, M. A. (1998). Language within our grasp. Trends in Neurosciences, 21(5), 188-194.

Rizzolatti, G., \& Sinigaglia, C. (2008). Mirrors in the brain: How our minds share actions, emotions, and experience (Translated from the Italian by Frances Anderson). Oxford: Oxford University Press.

Romanski, L. M., Tian, B., Fritz, J., Mishkin, M., Goldman-Rakic, P. S., \& Rauschecker, J. P. (1999). Dual streams of auditory afferents target multiple domains in the primate prefrontal cortex. Nat Neurosci, 2(12), 1131-1136.

Saur, D., Kreher, B. W., Schnell, S., Kummerer, D., Kellmeyer, P., Vry, M.-S., . . Weiller, C. (2008). Ventral and dorsal pathways for language. Proceedings of the National Academy of Sciences of the United States of America, 105(46), 18035-18040.

Sauser, E. L., \& Billard, A. G. (2006). Parallel and distributed neural models of the ideomotor principle: an investigation of imitative cortical pathways. Neural networks : the official journal of the International Neural Network Society, 19(3), 285-298. doi: 10.1016/j.neunet.2006.02.003

Saussure, F. (1916). Cours de linguistique générale (ed. by C. Bally and A. Sechehaye, with the collaboration of A. Riedlinger). Lausanne and Paris: Payot (English translation by W. Baskin, Course in General Linguistics, Glasgow: Fontana/Collins, 1977).

Schaal, S., Ijspeert, A., \& Billard, A. (2003). Computational approaches to motor learning by imitation. Philosophical Transactions of the Royal Society B: Biological Sciences, 358(1431), 537-547.

Segaert, K., Menenti, L., Weber, K., Petersson, K. M., \& Hagoort, P. (2012). Shared Syntax in Language Production and Language Comprehension-An fMRI Study. Cerebral Cortex, 22(7), 1662-1670. doi: 10.1093/cercor/bhr249

Senghas, A., Kita, S., \& Özyürek, A. (2004). Children Creating Core Properties of Language: Evidence from an Emerging Sign Language in Nicaragua. Science, 305, 1779-1782.

Sherman, J. C., \& Schweickert, J. (1989). Syntactic and semantic contributions to sentence comprehension in agrammatism. [10.1016/0093-934X(89)90029-1]. Brain and Language, 37(3), 419-439.

Spranger, M. (2014). The evolution of grounded spatial language. Berlin: Language Science Press.

Stokoe, W. C. (2001). Language in Hand: Why Sign Came Before Speech. Washington, DC: Gallaudet University Press.

Sutton, R. S., \& Barto, A. G. (1998). Reinforcement Learning: An Introduction. Cambridge, MA: The MIT Press.

Tagamets, M. A., \& Horwitz, B. (1998). Integrating electrophysiological and anatomical experimental data to create a large-scale model that simulates a delayed match-to-sample human brain imaging study. Cereb Cortex, 8(4), 310-320.

Tagamets, M. A., \& Horwitz, B. (2000). A model of working memory: bridging the gap between electrophysiology and human brain imaging. Neural Netw, 13(8-9), 941-952.

Tanenhaus, M. K., Spivey-Knowlton, M. J., Eberhard, K. M., \& Sedivy, J. C. (1995). Integration of visual and linguistic information in spoken language comprehension. Science, 268(5217), 1632-1634. doi: 10.1126/science.7777863

Tomasello, M. (1999). Emulation learning and cultural learning. Behavioral and Brain Sciences, 21, 703-704. 
Tomasello, M. (2003). Constructing a Language: A Usage-Based Theory of Language Acquisition. Cambridge, MA: Harvard University Press.

Tomasello, M., \& Call, J. (1997). Primate Cognition. New York: Oxford University Press.

Ueno, T., Saito, S., Rogers, T. T., \& Lambon Ralph, M. A. (2011). Lichtheim 2: Synthesizing Aphasia and the Neural Basis of Language in a Neurocomputational Model of the Dual Dorsal-Ventral Language Pathways. [10.1016/j.neuron.2011.09.013]. Neuron, 72(2), 385-396.

Umilta, M. A., Kohler, E., Gallese, V., Fogassi, L., Fadiga, L., Keysers, C., \& Rizzolatti, G. (2001). I know what you are doing. a neurophysiological study. [Research Support, NonU.S. Gov't]. Neuron, 31(1), 155-165.

Visalberghi, E., \& Fragaszy, D. (2001). Do monkeys ape? Ten years after. In K. Dautenhahn \& C. Nehaniv (Eds.), Imitation in Animals and Artifacts (pp. 471-500). Cambridge, MA: The MIT Press.

Vosse, T., \& Kempen, G. (2000). Syntactic structure assembly in human parsing: a computational model based on competitive inhibition and a lexicalist grammar. Cognition, 75(2), 105-143.

Waddington, C. H. (1953). The" Baldwin Effect"," Genetic Assimilation" and" Homeostasis. Evolution, 7(4), 386-387.

Weiller, C., Musso, M., Rijntjes, M., \& Saur, D. (2009). Please don't underestimate the ventral pathway in language. [10.1016/j.tics.2009.06.007]. Trends in Cognitive Sciences, 13(9), 369-370.

Werbos, P. J. (1990). Backpropagation through time: what it does and how to do it. Proc IEEE, 78(10), 1550-1560.

Wohlschläger, A., Gattis, M., \& Bekkering, H. (2003). Action generation and action perception in imitation: an instance of the ideomotor principle. Phil. Trans. R. Soc. Lond., 358, 501515.

Wray, A. (1998). Protolanguage as a holistic system for social interaction. Language \& Communication, 18, 47-67. 